\title{
ANTERIOR FONTANELLE PRESSURE MONITORING IN INFANTS
}

Fontaneldrukmeting bij jonge kinderen 
Vormgeving : Th. Vanoordt, Amsterdam.

Druk- en zetwerk : Roos en Roos drukkers, Arnhem.

\section{CIP-GEGEVENS KONINKLIJKE BIBLIOTHEEK, DEN HAAG}

Overweg-Plandsoen, Wouterina Cornelia Geertruida

Anterior fontanelle pressure monitoring in infants / Wouterina Cornelia Geertruida OverwegPlandsoen. - [S.l. : s.n.]. - Ill.

Proefschrift Rotterdam. - Met lit. opg. - Met samenvatting in het Nederlands.

ISBN 90-9003637-7

SISO 605.2 UDC 611.7:611.91/.93-052-053.2(043.3)

Trefw.: fontaneldrukmeting / kindergeneeskunde.

Copyright (C) 1990 by W.C.G. Overweg-Plandsoen

All rights reserved. No part of this publication may be reproduced, stored in a retrieval system, or transmitted, in any form or by any means, electronic, mechanical, photocopying, recording or otherwise without the prior permission of the author.

Adress: Academic medical centre, Department of child neurology G8-205, Meibergdreef 9, 1105 AZ Amsterdam, The Netherlands. 


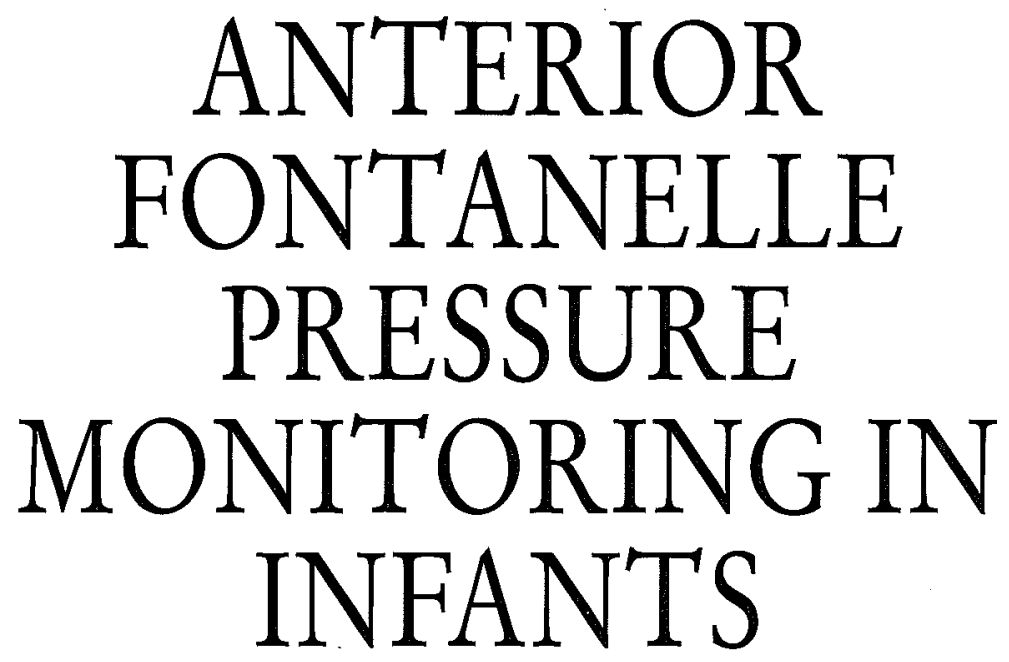

Fontaneldrukmeting bij jonge kinderen

\author{
proefschrift
}

ter verkrijging van de graad van doctor

aan de Erasmus Universiteit Rotterdam

op gezag van de rector magnificus

prof.dr. C.J. Rijnvos

en volgens besluit van het college van dekanen.

De openbare verdediging zal plaatsvinden op

woensdag 3 oktober 1990

om 15.45 uur precies

door

Wouterina Cornelia Geertruida Overweg-Plandsoen

geboren te Rotterdam 
Promotiecommissie

\section{Promotor}

Prof.dr. C.J.J. Avezaat

Co-promotor

Dr. M.C.B. Loonen

Overige leden

Prof.dr. H. van Crevel

Prof.dr. P.J.J. Sauer

Prof.dr.ir. C.J. Snijders 


\section{Meten is weten}

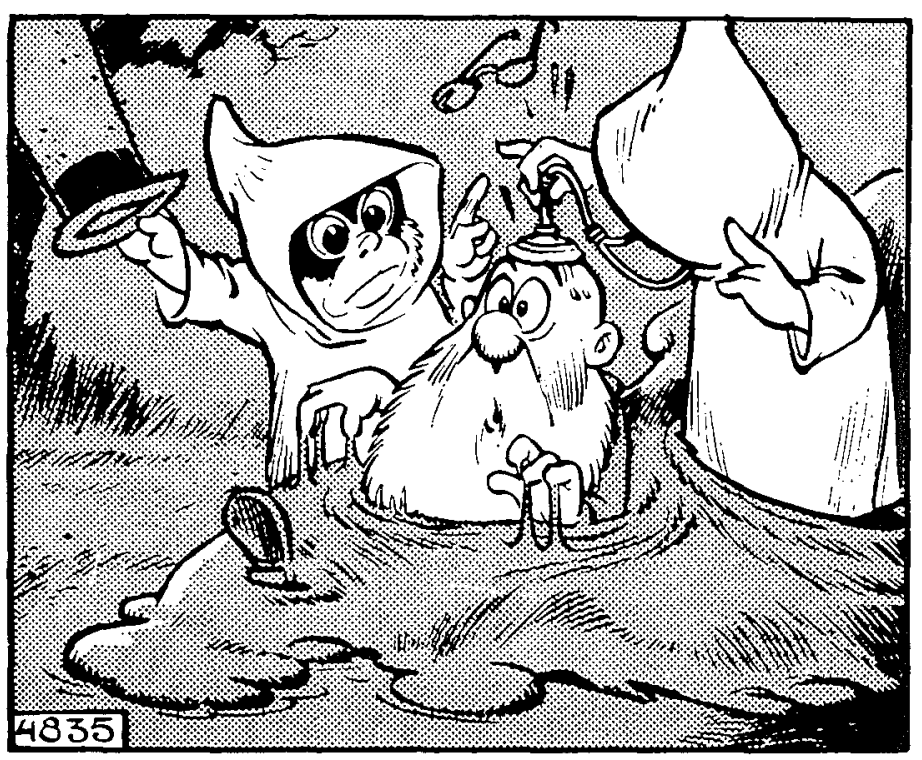

Voor alle kinderen die zich hebben laten meten 
Het verschijnen van dit proefschrift in deze vorm werd mogelijk gemaakt door steun van bet Van Leersumfonds, Katwijk Farma BV, Hoechst, Wellcome, Sanofi, Nourypharma en bet R.A. Laanfonds. 


\section{CONTENTS}

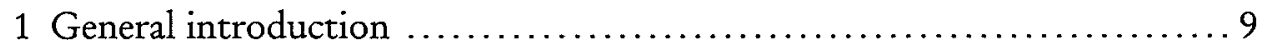

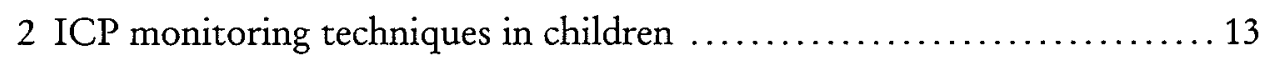

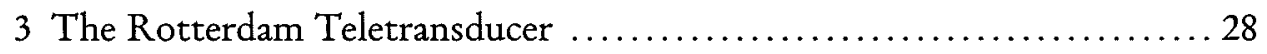

4 Anterior Fontanelle Pressure in normal newborns

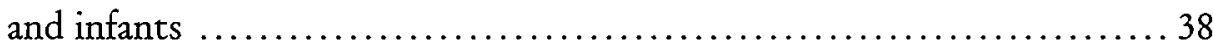

5 Anterior Fontanelle Pressure measurements in patients .............. 52

6 Inter-observer analysis in a random sample of Anterior

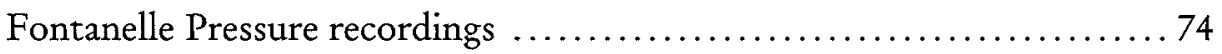

7 Anterior Fontanelle Pressure monitoring in clinical practice .......... 81

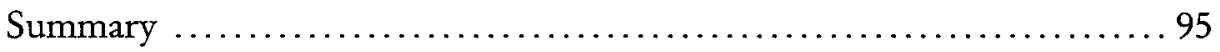

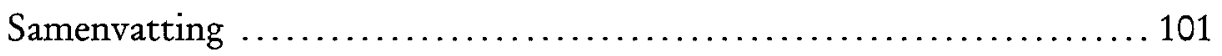

References .............................................. 106

Abbreviations ................................................ 120

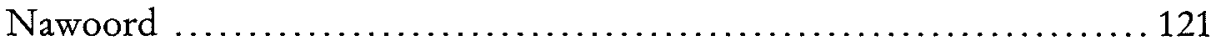

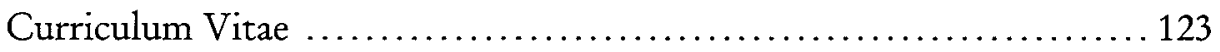





\section{H A P T E R 1 \\ GENERAL INTRODUCTION}

The idea of measuring intracranial pressure (ICP) dates back to 1866 when Leyden attempted to measure the ICP directly via a trephined opening. With the introduction of the lumbar puncture by Quincke in 1891 the possibility of examining the cerebrospinal fluid (CSF) and of measuring the cerebrospinal fluid pressure (CSFP) was born.

When continuous recording of ICP became possible in clinical practice (Guillaume and Janny 1951, Lundberg 1960) a new dimension was given to clinical research in the field of intracranial hypertension. Since then a continuous flow of papers on ICP has appeared in the international journals and a cycle of, so far, seven international symposia on this subject has been held between 1972 and 1988; the eighth symposium to come in 1991.

For a long time the diagnosis of 'normal', 'low', or 'high' ICP was mainly based on the presence or absence of certain neurological symptoms and signs in patients. Neurologists and neurosurgeons especially, were aware of the fact that intracranial pressure could influence cerebral function. This was based mainly on clinical observations and upon sporadically performed animal experiments.

Due to the extensive work of Lundberg (1960) more insight was gained into the variations of ICP in normal and pathological conditions. With the routine use of ventricular catheters to measure ventricular fluid pressure (VFP) it was possible to detect pathological alterations in the level of ICP, before clinical signs in- dicating a disturbance in cerebral function, appeared. Lundberg (1960) regarded the normal range of VFP as between 0 and $15 \mathrm{mmHg}$. Welch (1980) has more recently pointed out that in infants, an upper level of $5 \mathrm{mmHg}$ would be more appropriate. A mean ICP in excess of $20 \mathrm{mmHg}$ is agreed to be abnormal.

The role of (continuous) ICP monitoring in clinical practice is still open to discussion (Miller 1985, Ropper 1985). Its proponents claim that the technique is an integral part of neurologic and neurosurgical intensive care and should play a part in the management of every comatose patient, while opponents hold that there is no evidence that the implementation of ICP monitoring influences outcome to an extent that justifies the risks of this invasive procedure.

Many former beliefs about the clinical features of raised ICP are erroneous. The symptomatology of raised ICP depends as much on its cause as the level of pressure attained. In benign intracranial hypertension, for instance, ICP may be in excess of $60 \mathrm{mmHg}$, yet the patient may remain conscious with no abnormal neurologic signs, however, papilloedema is usually present (Grant 1971, Van Crevel 1982). In the patient with a head injury, an increase in ICP to $30 \mathrm{mmHg}$ may be associated with a marked reduction in the consciousness level with dilatation of a pupil and alterations in arterial pressure and heart rate. The only way to exclude raised ICP is to measure it. Raised ICP 
(a mean of more than $20 \mathrm{mmHg}$ ) occurs in $50 \%$ of all comatose patients with head injury, while papilloedema is present in less than 5\% (Miller et al. 1981). It occurs in the majority of comatose patients with subarachnoid haemorrhage or intracerebral haematoma and is common in patients who are comatose as a result of hypoxia, meningitis and encephalitis, toxic encephalopathies and even hepatic coma (Lundberg 1972, Moss and McDowall 1979, Brock 1983, Miller 1985).

The higher the ICP, the poorer is the prognosis. This is most clearly seen in patients with head injuries (Miller 1985). The most extreme example is where ICP rises to the level of arterial pressure, perfusion of the cerebral hemispheres and upper brainstem ceases and clinical brain death occurs.

Additional indications of the desirability of measuring ICP exist in patients with papilloedema of unknown origin or in patients with normal pressure hydrocephalus where it is important to know whether ICP is normal or increased (Hoffman 1986, Börgesen and Gjerris 1982). In cases of hydrocephalus ICP recording may be used for pre-operative assessment and also to check the effect of a shunt operation (Symon and Dorsch 1975). In the postoperative management of neurosurgical patients ICP monitoring may help in the early detection of a developing haematoma (Tagaki et al. 1986).

In pediatric patients, besides the above mentioned criteria for measuring ICP, special indications exist such as intraventricular haemorrhage in prematures and newborns (Ahmann et al. 1980, Dykes et al. 1989), (posthaemorrhagic) hydrocephalus (Hayden et al. 1970, Shapiro et al. 1984, Volpe 1989a,b), bacterial meningitis (Minns and Engleman 1988), and hypoxicischaemic encephalopathy secondary to cardiac arrest, strangulation or neardrowning (Donn and Philip 1978, Dean and McComb 1981, Levene and Evans 1983, Nussbaum and Galant 1983, Sarnaik et al. 1985).

It is important to have accurate information on the ICP. Besides the ventricular catheter (Lundberg 1960) other methods for measuring ICP were developed. Subarachnoid, subdural, intraparenchymal and epidural devices were introduced. Nowadays, the subarachnoid screw (Vries et al. 1973), the intraparenchymal fiber-optic transducer tipped catheter (Ostrup et al. 1987) and (telemetric) epidural transducers (De Jong et al. 1975, 1979, 1982, Kosteljanetz et al. 1986, Nornes and SerckHansen 1970, Tindall et al. 1972, Chapman et al. 1980) are being used in neurosurgical practice. However, leakage of CSF, blockage of a catheter or screw by swollen brain tissue, or errors in positioning and calibration of the transducer can contribute to an inaccurate record of ICP. Furthermore, physiologic parameters might influence ICP such as the systemic arterial pressure and respiration. Also intracranial pressure monitoring is invasive and introduces the risk of infection (Smith and Alksne 1976).

It is essential to balance benefit and risk in every patient in whom an ICP monitoring technique is used. There is no definite evidence that ICP monitoring will improve outcome, the only exception being perhaps in the management of Reye's syndrome in children. In this condition there is diffuse swelling of the brain and raised ICP that in some cases ends in brain death (Mickell and Ward 1984). In the management of patients with head injury it is difficult to prove that ICP monitoring improves outcome or mortality (Miller 1985, Stuart et al. 1983). 
ICP monitoring can aid management. It provides the only way to confirm or exclude intracranial hypertension. It can help in assessing whether therapeutic interventions to lower pressure are effective (Lundberg et al. 1974, Miller 1983, 1985, Miller et al. 1977, Narayan et al. 1982). If the patient is heavily sedated or paralyzed and is artificially ventilated measuring ICP provides the only means to monitor the patient's cerebral condition.

Although techniques for monitoring ICP could be adjusted for use in children (Hayden et al. 1970, James et al. 1975) most clinicians are reluctant to use invasive measuring techniques routinely in younger children because of the infection risk and of the technical problems, especially when longer monitoring periods are necessary. Non-invasive measurement techniques would be ideal, however in patients with closed sutures there is no possibility of doing so. In children with an open fontanelle there is a natural access to the intracranial compartment and therefore anterior fontanelle pressure (AFP) monitoring would be ideal.

The oldest method for estimating noninvasively the ICP in infants is the palpation of the anterior fontanelle. Although frequently used by clinicians treating children, this subjective method is not considered a very reliable method of quantifying the pressure level. Much effort was expended on developing non-invasive methods for measuring ICP accurately via the anterior fontanelle. Physicists and clinicians turned out to be geniuses in developing a large number of different measuring devices for AFP monitoring, each with its own specific technical and clinical problems. The fixation of a device on the child's head is not very simple, not only during short term measurements but especially during continuous AFP monitoring.
Furthermore, the fixation method tends to influence the actual pressure recording as a result of application forces.

In the experimental neurological and neurosurgical department of the University Hospital of Rotterdam (Dijkzigt) an implantable, stable, telemetric transducer with a fast dynamic response had been developed for epidural pressure (EDP) measurements in adult patients (De Jong et al. 1975, 1979, 1982). It was tempting to investigate whether this Rotterdam Teletransducer (RTT) could also measure AFP in infants. However, a fixation method had to be developed in order to avoid application forces and to provide for an accurate pressure recording.

\section{The objectives of this study were}

1 To evaluate whether the RTT could be used as a 'fontanometer'.

The literature on non-invasive ICP measurement techniques used in infants and children was studied to avoid problems already encountered by others. Subsequently, a special RT'T holding device with fixation frame was developed for the accurate measuring of AFP.

2 To test this measuring technique in a clinical setting AFP was established in healthy newborns and infants and the pressure values thus gathered were related to the normal values reported in the literature. Thereafter, AFP was monitored in patients with various neurological and pediatric diseases. The technique was tested both during short term and long term (continuous) measurements. The AFP recordings themselves, were tested on their readability by four observers by performing an inter-observer analysis.

3 To test whether the results of AFP measurements could have played a role in clinical practice with respect to the question of whether or not shunting operations 
should be performed, a retrospective study of the patient data was undertaken.

In the following six chapters of this thesis these objectives will be clarified. After a historical review (chapter 2) the AFP monitoring technique will be described (chapter 3). Normal AFP values and AFP values in patients will be given and discussed in the chapters 4 and 5 respectively. An interobserver study of the AFP recordings including statistical analysis procedures is the subject of chapter 6 . In chapter 7 the role of AFP monitoring in daily clinical practice will be viewed in the aspect of performing shunting operations in children. 


\section{ICP MONITORING TECHNIQUES IN CHILDREN}

\section{INTRODUCTION}

By the end of the nineteenth century Quincke (1878) reported on 'the pressure measured in a meningocele as big as an apple located in the lumbar region in an infant of 11 weeks old'. With the child lying in the lateral recumbent position, he measured the pressure by stinging a hollow needle into the cele. This needle was connected through a short rubber tube with an open glass pipe kept in vertical position. The level of the fluid column (cm) was transferred mathematically into $\mathrm{mmHg}$. The pressure thus measured was $4 \mathrm{mmHg}$.

In 1891(b) Quincke reported the technique of the spinal tap in the lumbar region 'with a thin hollow needle beneath the arch of the 3 rd and 4th lumbar vertebra'. He described thoroughly how a lumbar puncture had to be performed with the patient in the lateral recumbent position, the legs flexed at the hips and the head slightly bent forward. Quincke drew attention to the change in the anatomical relations of the lumbar spine with increasing age, this having implications for the exact site and the direction of the puncture. In another report (1891a) he described how he performed multiple lumbar punctures in 5 children, 3 children suffering from hydrocephalus and 2 children from meningoencephalitis. He gives the noteworthy history of a 15 months old girl suffering from a chronic hydrocephalus with an acute exacerbation and every clinical sign of increased intracranial pressure (apathy, macrocephaly, venous congestion of the skull, a large tense fontanelle, opisthoto- nus and occasional vomiting). After a lumbar puncture and the evacuation of 12 cc of CSF the child's condition improved and the fontanelle was less tense. In spite of multiple lumbar punctures the child could not be cured and after nine days she died from rising ICP, high temperature and seizures. The clinical diagnosis of hydrocephalus was confirmed at autopsy. Since this first description, the lumbar puncture technique has been routinely used in neurological and pediatric practice, not only for measuring the pressure of the cerebrospinal fluid but also for examining its constituents.

Other invasive techniques were developed for measuring ICP in patients with closed sutures, both adults and children (Lundberg 1960, Newton 1987). These techniques can be classified according to the recording site.

- CSF pressure by lumbar-, cisternal- or ventricular puncture (Quincke 1891a,b, Levinson 1928, Munro 1928, Hayden 1968, 1970, De Lange et al. 1968, Paraicz and Vajda 1975a, Minns 1976, 1977, 1984, McCullough 1980),

- Pressure at the surface of the brain by subdural or subarachnoid catheters or a subarachnoid screw (James et al. 1975, Levin 1977, Ivan et al. 1980, Roberts 1983, Levene and Evans 1983, 1985, Landy and Villanueva-1984, Kaiser and Whitelaw 1985, 1986),

- Intraparenchymal pressure by inserting a transducer tipped catheter into the brain 


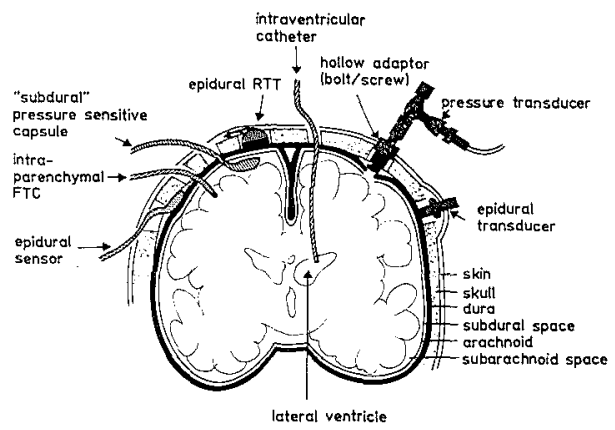

Fig. 1 Schematic representation of the intracranial pressure measurement sites and sensors. RTT $=$ Rotterdam Teletransducer. FTC $=$ fiber-optic transducer tipped catheter.

(Ostrup et al. 1987, Hollingworth et al. 1988).

- Epidural pressure by means of several kinds of pressure sensors (Levin 1977a,b, Ivan et al. 1980, Bada 1983).

The sites for recording ICP (Allen 1986) are schematically represented in figure 1. Clinicians treating children meet specific problems in ICP monitoring. The size of commercially available devices is, generally speaking, too big to use on children, necessitating adaption of the devices. Measuring systems for children have to be more flexible than the ones used in adult patients. Also the fixation of the system is very important since most children do not automatically co-operate and lie still. Most clinicians are reluctant to use invasive monitoring techniques in young children mainly because of the infection risk and the technical problems. Since the anterior fontanelle affords a natural access to the intracranial compartment, devices were developed for measuring ICP noninvasively via the anterior fontanelle.

ICP monitoring can be performed for periods ranging from a few minutes to many days. Whether short term or long term (continuous) measurements will be employed depends on the clinical problem, the time necessary to record certain phenomena and on the interest of the individual investigator.

In this chapter the several techniques for measuring ICP invasively as well as noninvasively will be described. Attention will be given to the advantages and the disadvantages of the several techniques from both a clinical and a technical point of view.

\section{DESCRIPTION AND DISCUSSION OF METHODS}

\section{CSF pressure}

\section{Lumbar puncture}

This most often used routine procedure has several advantages. It is easily performed, venting of CSF is possible when a free communication between intracranial space and spinal canal exists, chemical, cytological and bacteriological analysis of CSF is possible, and also drugs can be administered. By inducing with a constant rate fluid into the cerebrospinal fluid compartment (infusion test) the outflow resistance (Ro) can be measured, this being described by Portnoy and Croissant (1978), Sklar et al. (1980) and Avezaat and Van Eijndhoven (1984). Calibration of the system is easily done in situ. The level of the external transducer has to be at the level of the right atrium (zero point). A disadvantage of this method is the danger of brainstem herniation in case of obstructed CSF pathways. Continuous monitoring of more than 24 hours is only possible by using a catheter.

CSF pressure measured by cisternal puncture is comparable to the lumbar puncture. 
However, the anatomic relations of the brainstem and the cervical spinal canal should be thoroughly born in mind to prevent lethal complications.

\section{Intraventricular Pressure recording}

Originally an indwelling ventricular catheter, inserted through a burrhole in the frontal horn of one of the lateral ventricles was used (Lundberg 1960). The catheter was connected to an externally mounted sensor by means of a fluid filled transmission line, with adequate sealing of all tube connectors to prevent leakage of CSF. This method for measuring VFP is still considered the 'golden standard' for ICP monitoring and serves as a reference for other ICP recording techniques.

The advantages of the method are comparable to those mentioned under the lumbar puncture technique. Biochemical, cytological and bacteriological analysis is possible (Turner and McDowall 1976) and drainage of CSF to lower ICP is feasible without the danger of brainstem herniation (Jennett 1977). Drugs can be administered (e.g. antibiotics) when a rapid concentration and action is desirable (Lundberg et al. 1974).

The volume-pressure test, developed by Miller et al. (1973), can be performed to measure the elastance $\mathrm{E}(\mathrm{dP} / \mathrm{dV})$ or the compliance $\mathrm{C}(\mathrm{dV} / \mathrm{dP})$ of the craniospinal compartment. This test involves the rapid injection or withdrawal within one second of $1 \mathrm{ml}$ of saline into or from the lateral ventricle, whereafter changes in ICP are measured.

Calibration of the system is easily done in situ. This method is suitable not only for short term but also for continuous ICP monitoring. However, the method has some disadvantages. There is a risk of intracranial infections, especially after measurements lasting longer than three days (Rosner and Becker 1976, Weinstein 1976). The infection rates reported, range from $3.5-6 \%$ up to $12 \%$ (Lundberg 1960, Sundbärg et al. 1972, Wyler and Kelly 1972, Rosner and Becker 1976, Smith and Alksne 1976). When the ventricles are compressed or displaced difficulties may arise in the proper placement of the catheter. Intracranial haematoma (Miller 1978, Fleischer et al. 1976), and epileptic seizures (Miller et al. 1977) may be induced. The method is unsuitable for outpatient monitoring.

\section{Pressure at the surface of the brain \\ Subarachnoid ICP recording}

Vries et al. (1973) designed a hollow screw which could be easily inserted, under local anaesthesia, into a burrhole in the skull, projecting through the dura into the subarachnoid space. By means of a saline filled extension tube it was connected to an externally located transducer. A modified (smaller) screw for pediatric use was developed by James et al. $(1975,1976)$ and Landy and Villanueva (1984). Dearden et al. (1984) introduced the Leeds bolt with multiple side holes to minimize obstruction by blood clots. The advantages of this method are the relative ease of placing the device at the bedside under local anaesthesia without the risk of brainstem herniation in case of obstructed CSF pathways and the possibility of calibration in situ. The disadvantages of this method are the infection risk, though lower than in the case of intraventricular pressure recordings (Winn et al. 1977), or subdural haematoma resulting from a bleeding caused by incision of the dura, especially in blood coagulation disorders (Vries et al. 1973). Usually, venting of CSF is not possible and the method is not suitable for out-patient monitoring. Blockage of the adaptor by meninges or a blood clot 
or debris, especially at high levels of ICP, may result in underreading of ICP (Rowan et al. 1983, Mendelow et al. 1983). To avoid this, the system can be flushed although this might induce high intracranial pressure waves and might increase the infection rate.

\section{Subdural ICP recordings}

Initially, pressure sensors for subdural insertion consisted of fluid filled balloons or tambours connected to an externally located transducer through a fluid filled tubing (Rothballer 1963, Hoppenstein 1965). Thereafter, a so called switch was introduced, consisting of a $9 \mathrm{~mm}$ diameter silastic envelope enclosing two gold electrodes and the poles of a single switch connected externally by wires passing through polyvinyl tubing. The internal pressure introduced via the tubing, being just sufficient to separate the contacts and thus opening the switch was considered equal to the external (intracranial) pressure (Numoto et al. 1966). Later on other devices were developed consisting of transducers based on the principle of a Wheatstone bridge, in which a change in resistance is converted into a pressure analogous voltage; for instance strain gauge transducers like the Gaeltec pressure monitor (Roberts et al. 1983). Several materials were used, like beryllium-copper (Jacobson and Rothballer 1967), silver (Nornes and Serck-Hansen 1970) and titanium (Tindall et al. 1972). The authors encountered several problems with these devices like temperature sensitivity (Coe et al. 1967), leakage of CSF into the device (Jacobson and Rothballer 1967), zero drift (Nornes and Serck-Hansen 1970, Tindall et al. 1972) and calibration difficulties (Heppner et al. 1976, Roberts et al. 1983).

The sizes of the transducers varied from $2 \times 4.5 \mathrm{~mm}$ to $14 \times 12 \mathrm{~mm}$. All transducers had wired connections to externally located pressure recorders.

Although this method gives a reduction of the infection rate compared to VFP recordings, infectious complications may arise e.g. subdural empyema (Rand et al. 1966, Kindt 1975). Since the transducers are located at the measuring site a reference point is less important than in CSFP monitoring. Furthermore, the dura characteristics do not interfere with the pressure recording. The disadvantages are the impossibility of venting CSF, the risk of subdural haematoma (Rand et al. 1966) and some difficulty in insertion. Subdural pressure, compared to VFP was tested with the Gaeltec transducer (Barlow et al. 1986) and tended to be slightly higher. For out-patient monitoring the method is not suitable. Depending on the type of device there may be zero drift and usually recalibration in situ is not possible. Furthermore, the high costs of most of the devices are a limiting factor for routine clinical use (Roberts et al. 1983).

\section{Intraparenchymal ICP recor- ding}

This method has been advocated with the introduction of a fiber-optic transducer tipped catheter (FTC), the so called ' $\mathrm{Ca}$ mino' catheter. To measure ICP the catheter is inserted, through a subarachnoid bolt, several centimeters beyond the bolt. In an animal model and later in patients, brain tissue pressure was slightly higher than intraventricular pressure. Pressure changes were recorded adequately (Ostrup et al. 1987, Hollingworth et al. 1988). The advantages of the method (and the device) are the relative ease of insertion of the transducer. The transducer has a fast response, so rapid pressure fluctuations can be recorded. The disadvantages so far are the risks of infection and haematoma. Furthermore, there is no possibility of 
venting CSF and no possibility for recalibrating if there is a zero drift.

\section{Epidural ICP recording}

During the past 15-20 years this method has been advocated more and more since the dura does not have to be opened for implanting a pressure sensor, so that the brain's natural barrier against detrimental influences from outside remains intact.

There are two ways of applying a sensor: non-co-planar to the dura or according to the co-planarity principle. The latter method is considered superior because the tension forces of the dura are almost completely eliminated (Mackay et al. 1960, Mackay 1964, Schettini et al. 1971, Schettini and Walsh 1974, 1975).

\section{Non-co-planar sensors}

The several types of devices to be described, are inserted through a burrhole in the skull stripping off the underlying dura over a certain area (Gobiet et al. 1972). Initially, bags (Numoto et al. 1973) or capsules (Coroneos et al. 1973, Turner et al. 1975) or cups (Kosteljanetz et al. 1986), connected to an externally located transducer were developed. Subsequently, devices were constructed according to the Wheatstone bridge principle, e.g. strain gauge transducers (Dorsch et al. 1971, Roberts et al. 1983) or transducers with a movable silicon rod (Nornes and Magnaes 1971). Another principle used has been the pneumatic flow switch (Levin et al. 1983b, Powell and Crockard 1985). For all these sensors a wired connection with the outside world was needed. With the introduction of passive devices, consisting of an inductance and a pressure sensitive capacitance resonance at specific frequencies varying from 50 to $100 \mathrm{~Hz}$ (Gücer et al. 1979, 1980, De Jong et al. 1982) it became possible to measure pressure telemetrically without direct contact between the transducer and the electronic equip- ment, thereby reducing the infection risk. A sensing coil at a certain distance from the transducer radiates a radiofrequency specific to the device. At the resonance frequency of the transducer the energy is absorbed, this being detected by the external sensing coil, whereafter the external equipment converts the 'absorption frequency' into an indication of pressure. The zero drift tendency of the devices decreased after making them impervious to water. It was, therefore, important to choose the correct materials for constructing the sensors (Nornes and Magnaes 1971, Gücer et al. 1980, De Jong et al. 1979, 1982).

The size of the described sensors varied from $2 \times 4.5 \mathrm{~mm}$ to $12.5 \times 8 \mathrm{~mm}$.

\section{Co-planar sensors}

In order to measure intracavitary pressure accurately it is necessary that the bending forces of the covering membrane(s) are eliminated (or at least reduced). Kleiber (1945) described a tympanometer for measuring intra-abdominal gas in cows. Smyth (1957) described a guard-ring tocodynamometer for measuring intraamniotic pressure through the intact abdominal wall. Smyth (1957) and, in more detail, Mackay and co-workers (1960, 1964) demonstrated that this intracavitary pressure was accurately measured if the bending or tension forces of the covering membrane were absorbed at the periphery of the pressure sensing device. This could be accomplished by flattening the membrane, thereby reducing the membrane tensile or stretching forces to zero. This so called 'aplanation' principle was used in ophthalmology for measuring intraocular pressure (Schulz et al. 1963, West et al. 1972). This principle was also used for measuring intracranial pressure through an intact dura (Schettini et al. 1971, Majors et al. 1972). 


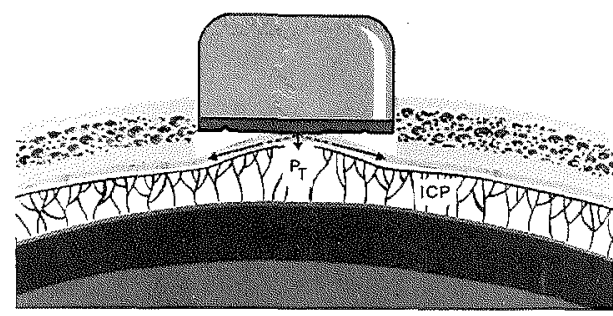

Fig. 2 Schematic representation of a transducer in a burrhole in the skull. The dura tensile forces result in $\mathrm{P}_{\mathrm{T}}$. The pressure acting below the dura is equal to ICP. The tensile forces and the ICP cause the dura to expand. There will be an error in measurement because $P_{T}$ and ICP are acting in opposite directions.

When a transducer is pressed against the dural membrane when it is slack, it measures the forces exerted by the underlying matter. The surface pressure upon the transducer can be calculated if the area of the transducer is known. When the dura is under tension and the same transducer is applied against its surface, there will be an error in measurement because two force components are now present: the dura stretching force (or tensile force) and the pressure acting beneath the dura (i.e. ICP). Figure 2 gives a schematic representation of an epidural transducer in a burrhole in the skull to demonstrate the several forces acting in epidural ICP monitoring.

The tensile force component can be alleviated if the following conditions are met. The transducer sensing surface must be flat and surrounded by a co-planar nonsensitive outer ring to provide a complete co-planar approximation with the dura. However, it should not be too flat (= stiff), because then it cannot measure anymore since 'measuring' means 'slightly moving with changing pressures'. With a co-planar approximation of the dura and the flat surface of the transducer, the

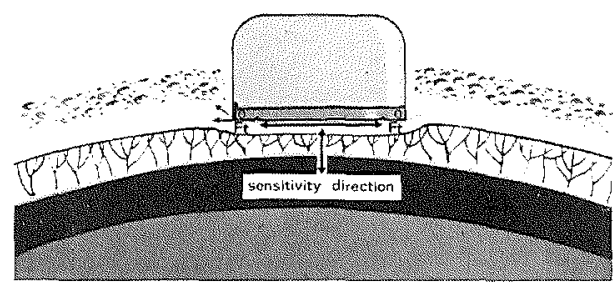

Fig. 3 Schematic representation of a transducer placed coplanar to the dura. Dura tensions $(\mathrm{Ft})$ are reduced to zero and are now perpendicular to the sensitivity direction of the transducer, therefore not influencing the pressure indication. The outer non-sensitive ring (o) sees to dissipation of the elastic (stretching) membrane forces.

instrument will measure the pressure beneath the dura regardless of dural tension, because the tensile forces $(\mathrm{Ft})$ are reduced to zero and the elastic forces (stretching forces) of the membrane are dissipated at the periphery of the outer non-sensitive ring. Figure 3 gives a schematic representation of this situation. A comparison can be made with a finger just touching the stretched membrane of a drum. If the pressure inside the drum is increased, the finger will readily detect the pressure change. However, if the membrane stretching is increased, the sensing finger cannot detect tension changes. The proper procedure for measuring EDP will be described in a separate paragraph on page 1921.

Various authors have reported on epidural transducers for co-planar application to the dura. Distinction can be made between transducers acting according to the Wheatstone bridge principle (e.g. strain gauge sensors) and devices acting according to the inductance-capacitance ( $\mathrm{L}-\mathrm{C}$ circuit) principle. For all types a classification can be made into active devices, meaning operating with built-in batteries 
and passive devices, meaning those operating batteryless. Strain gauge telemetric sensors were reported by Dorsch et al. (1971), Dorsch and Symon (1975) and Furuse et al. (1983). Shortly thereafter Beks et al. (1977) and Koster and Kuypers (1980) introduced the solid Philips transducer which was mounted in an adaptor for proper positioning into a burrhole in the skull. A transducer adapted for pediatric use was reported by Dietrich et al. (1977).

Epidural transducers acting according to an L-C circuit were reported by Atkinson et al. (1967), Brock and Diefenthäler (1972), Heppner et al. (1976), Rylander et al. (1976), Zervas et al. (1977), Fryer et al. (1978), Cosman et al. (1979) and De Jong et al. (1975, 1979, 1982). The materials used were copper-beryllium (Brock and Diefenthäler 1972), epoxy resin (Dorsch et al. 1971, Dorsch and Symon 1975), stainless steel (Atkinson et al. 1967, Koster and Kuypers 1980), teflon (Rylander et al. 1976) and titanium (De Jong et al. 1975, 1979). The problems encountered were zero drift due to leakage of CSF into the transducer, temperature sensitivity (Atkinson et al. 1967), no possibility for calibration in vivo, implantation failures due to non-exact co-planar positioning (Koster and Kuypers 1980), the rather big size of some of the devices, no availability of energy sources with sufficiently wide transmission range, and problems in finding proper biocompatible materials for constructing the devices. The size of the devices varied from $1.2 \times 1.2 \mathrm{~mm}$ to 14 $\mathrm{x} 12 \mathrm{~mm}$.

Summarizing, the main advantage of epidural ICP recording is the intact dura resulting in a reduced infection rate and with telemetric implantable transducers a still further reduction of infection risk. Furthermore, it is rather easy to place a sensor through a burrhole in the skull. There is a good correlation with VFP although with proper positioning of the transducer EDP tends to read slightly higher than VFP (Coroneos et al. 1973, Rylander et al. 1976, Powell and Crockard 1985), probably due to the characteristics of the dura. The disadvantage of this method is the risk of bad contact between the dura and the transducer if it is implanted obliquely, leading to erroneous ICP values. Furthermore, there is no possibility of removing CSF in order to decrease high ICP or to examine the CSF. Without telemetry the method is not suitable for out-patient monitoring. With telemetry however the method can be used on an out-patient basis.

Exact co-planar positioning of an epidural transducer is essential for reliable ICP monitoring. The method has been extensively investigated and described by Schettini et al. (1971) and will be described next. This technique has been used in the work leading to this thesis for measuring anterior fontanelle pressure (chapter 3 ) and therefore extra attention is paid to this aspect.

\section{Epidural ICP monitoring with the pressure depth curve}

Schettini and Walsh (1974) performed dynamic epidural pressure tests and gave a detailed description of how the subarachnoid and subpial compartments could be determined by means of an epidurally placed transducer. They performed the tests on anaesthetized dogs, after making a trephine opening in the skull and inserting a small transducer mounted in a piston and coupled to a plunger. A multiturn rotational potentiometer, geared to the plunger provided an accurate measure of the piston relative to the initial or reference position. For assuring continuous 


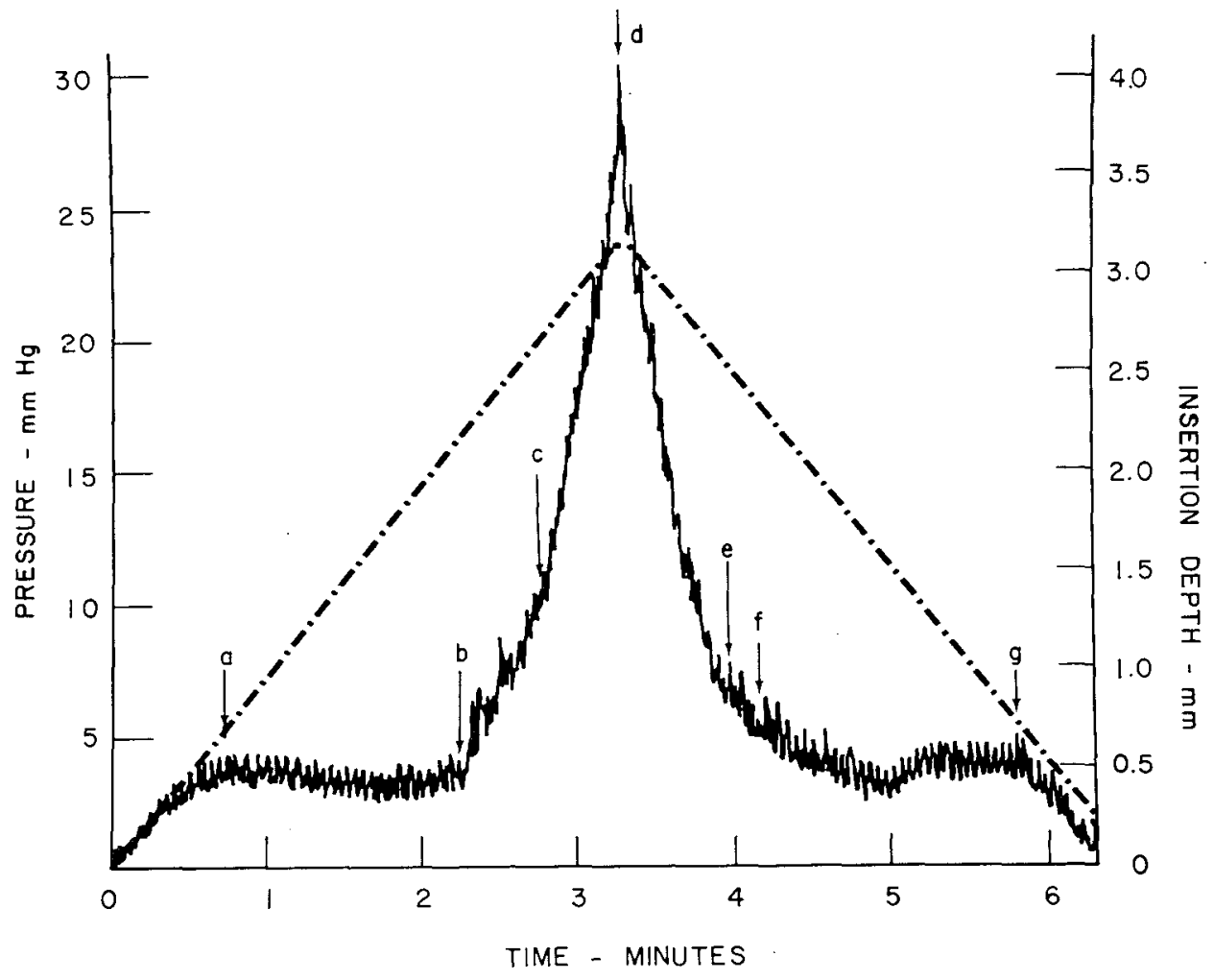

insertion and withdrawal at a constant rate, a gearmotor-drive system with manual and semi-automatic control was developed. The pressure test consisted of inserting a transducer at a constant rate to a preset pressure (usually $30 \mathrm{mmHg}$ ), followed by immediate withdrawal at the same rate. After each test the system was allowed to recover for a minimum resting interval of 15 minutes. The response of a typical pressure test is the so called Pressure Depth Curve (PDC). An example of a PDC is shown in figure 4.

The solid line indicates the pressure response and the broken line the corresponding displacement of the transducer. For both items the zero point represents initial dural contact. At point 'a' the transducer base is in complete contact with the dura and the pressure is essentially the CSF

Fig. 4 The pressure depth curve (PDC) as the result of a pressure test recording. The solid line is the pressure response $(\mathrm{mmHg})$; the broken line is the insertion depth $(\mathrm{mm})$. Zero point represents initial dural contact. See text. (Reproduced with permission, from Schettini A and Walsh EK 1974, J Neurosurg 40:609616).

compartment pressure. In this particular pressure response test the insertion depth at point ' $a$ ' was $0.6 \mathrm{~mm}$ and the pressure $3.5 \mathrm{mmHg}$. From point ' $a$ ' to ' $b$ ' the transducer traverses the subarachnoid CSF region, it does not encounter any opposing forces and a plateau appears where the pressure remains constant. At point 'b', at a depth of $2.2 \mathrm{~mm}$ and 4 $\mathrm{mmHg}$, the transducer is in contact with the subpial region. Continued insertion results in a pressure increase with a tran- 
sition point at point ' $c$ ', at $2.7 \mathrm{~mm}$ and $11 \mathrm{mmHg}$, representing substantial contact with the brain surface. That is the position beyond which insertion results in pressure changes associated primarily with brain compressing. Upon reaching point ' $\mathrm{d}$ ', at a depth of $3.1 \mathrm{~mm}$ and a pressure of $30 \mathrm{mmHg}$, the transducer is withdrawn at a rate equal to that of insertion. Point ' $\mathrm{d}$ ' thus represents the maximum insertion depth. On withdrawal the point corresponding to point ' $c$ ' during insertion, shown here as point ' $e$ ', is again at a depth of $2.7 \mathrm{~mm}$ but the pressure is $7.5 \mathrm{mmHg}$. The pressure at ' $e$ ' is lower than that at ' $c$ ', and this occurred with every test. The decrease in pressure is probably due to extra CSF absorption induced by the insertion of the transducer. Thus the pressure at a given insertion depth within the subpial region is less during withdrawal than during insertion.

Considering the various ICP measuring sites and the types of devices developed so far it can be concluded that the ideal transducer for measuring ICP should have as many as possible of the following characteristics: extradural positioning, no infection risk, providing comfort for patient and doctor, easy to implant or applicate, preferably no connecting wires to the outside world, small physical size, calibration capacity from outside, stability, minimal temperature sensitivity, imperviousness to the environment, extended use availability for a long period of time and reasonable costs.

Telemetry in the absence of any galvanic contact with the outside world together with a batteryless operated sensor, fulfilling all these requirements, would provide the ultimate safety of the patient. In chapter 3 an example of a transducer fulfilling many of these characteristics will be described.
After having described and discussed the various invasive ICP monitoring techniques the remaining part of this chapter will deal with non-invasive ICP monitoring techniques.

\section{Non-invasive ICP monito- ring techniques}

The oldest method for 'measuring pressure' in children with an open fontanelle is the palpation of the fontanelle. Clinicians dealing with children tend to judge certain aspects of the fontanelle such as the tension of the fontanelle and the presence of pulsations of the fontanelle. Although a clinician is able to discern gross changes in the tension of the fontanelle, there is such a variation in both the size and the fullness of the fontanelle that it is difficult to make an accurate prediction about the level of ICP. This subjective method of palpation is therefore considered unreliable. In one report fontanelle tension was assessed by palpation and related to invasively measured CSF pressure and considered not completely reliable (Kaiser and Whitelaw, 1987b). However, there has been attempts to classify certain aspects of the fontanelle. The level of the fontanelle varying with different positions of the child was related to hydrostatic pressure (Welch 1978, 1980). The zero point was that position in which the fontanelle was observed as flat, representing an equal level of pressures (ICP and atmospheric pressure) on both sides of the membrane. This method was also not very reliable since it was difficult to determine exactly when the fontanelle was flat, especially when the scalp was thickened by haemorrhage or oedema.

Attempts to measure ICP more accurately and non-invasively via the anterior fontanelle date from the beginning of this century when Wentzler (1922a,b) introdu- 
ced the so called 'tonometer for measuring the pressure on the skull of a newborn baby'. From that time onwards numerous reports have appeared recommending various devices for measuring 'fontanelle pressure' in infants.

The measuring devices can be divided into four categories according to the technical principles.

1 Weight controlled tonometers (Wentzler 1922a, Davidoff and Chamlin 1959, Edwards 1974).

2 Air or fluid filled bags or tambours (Purin 1964, Brett 1966, Blaauw et al. 1974, Hayashi et al. 1978).

3 Devices based on the (pneumatic) flow switch principle (Vidyasagar and Raju 1977, Hill and Volpe 1981a, Bunegin et al. 1987).

4 Transducers designed according to the Wheatstone bridge principle (Wealthall and Smallwood 1974, Hirsch et al. 1978, Huch et al. 1979, Peabody et al. 1981). Furthermore the measuring methods can be categorized.

1 Methods based on the pulsations of the fontanelle (Barashnev and Leontiev 1966, Picton-Warlow and Robinson 1970, Dietze et al. 1972, Blaauw et al. 1974).

2 Methods based on the tension of the fontanelle (Davidoff and Chamlin 1959, Wealthall and Smalwood 1974, Robinson et al. 1977, De Jong et al. 1984, Kaiser and Whitelaw 1987a, Mehta 1988).

For the purpose of clarity the several methods for measuring anterior fontanelle pressure will be discussed according to the basic measuring method (i.e. based on the pulsation or on the tension of the fontanelle). Specific problems like the fixation of the devices will be also dealt with.

For accurate anterior fontanelle pressure monitoring, besides the criteria mentioned on page 21 , some extra requirements are needed. The device should be small en- abling pressure measurements in a small fontanelle. The application of the device should not interfere with the monitoring of the actual pressure (see for explanation page 24), and the fixation of the device should not hinder the child from moving around freely. Furthermore, the fixation procedures should be suitable for long term ICP monitoring. These aspects will be discussed together with the various fontanelle pressure measuring devices.

\section{Methods based on the pulsations of the fontanelle}

In 1921(b) Wentzler patented his 'Tonometer for measuring intracranial pressure in babies'. The method was based on the principle of a weight controlled pin moving up and down with the natural pulsations of the fontanelle. These movements were amplified and transmitted onto an indicator via a toothed wheel. This indicator gave an indirect and only rough indication of pressure on a calibrated scale. In figure 5 this tonometer is shown.

Wentzler (1921b) related the maximum value of the indicator to the fontanelle size and concluded thereafter the pressure to be normal or pathological. It was important to keep the tonometer vertical on the fontanelle by means of a handle. A special leveling instrument was built in for checking the right position. The device had to be applied and held by hand and therefore successful measurements were not easily obtained.

An airfilled tambour (or vessel) for measuring anterior fontanelle pressure was introduced in the early sixties (Purin 1964, Brett 1966, Barashnev and Leontiev 1966). The device measured pulsations comparable to a device introduced earlier (Riechert and Heines 1950) for measuring dural pulsations through a burrhole of the skull. The tambour was placed on the bony 

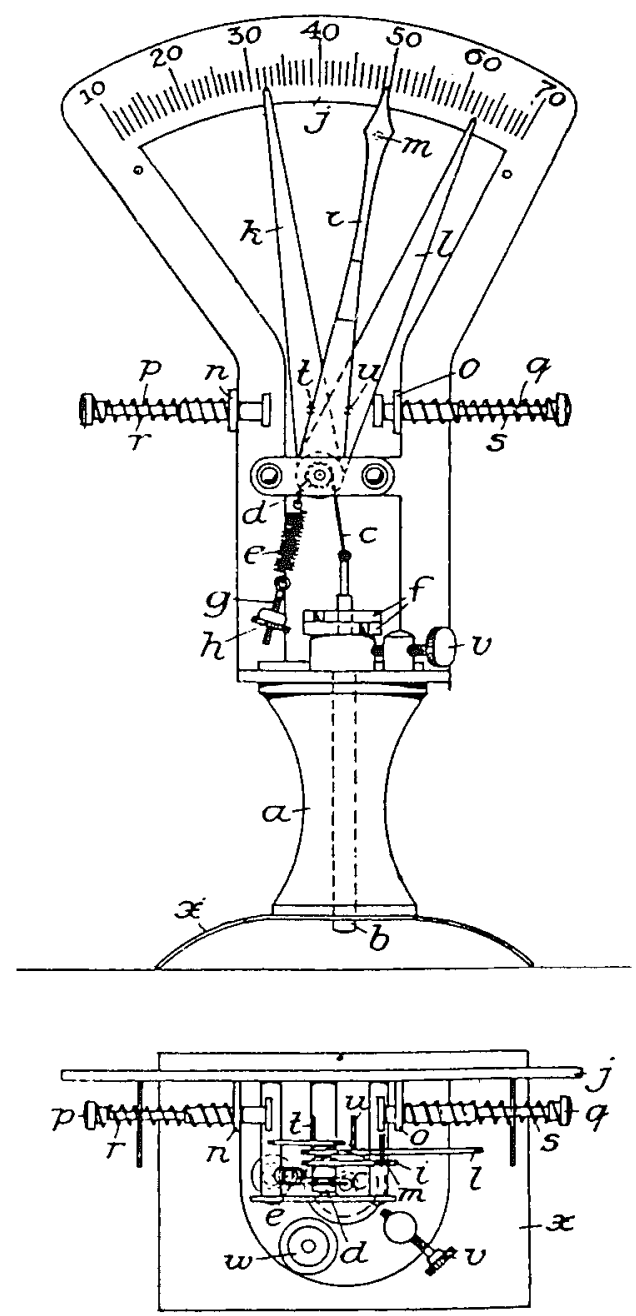

Fig. 5 Representation of the 'Tonometer for measuring intracranial pressure in babies', designed and patented by Wentzler in 1921. For the clarification the reader is referred to the original article.

margins of the fontanelle. Increasing the pressure in the tambour made the stretched membrane very sensitive to fontanelle pulsations and so small pulsations would show the greatest amplitude on the connected recorder. As the pressure in the system was slowly raised or lowered the amplitude of the recorded waves increased or decreased. The pressure recorded at the point of maximal amplitude was taken as corresponding to the CSF pressure in that area of the fontanelle to which the tambour was attached.

Picton-Warlow and Robinson (1970) published a critical study on the above mentioned apparatus and concluded that the method was not suitable, mainly because the membrane tensions fluctuated so much that no reliable results could be obtained. They concluded from a testmodel that the recorded pulsation amplitude was not at all related to intracranial pressure. On the contrary, the amplitude depended on the sensitivity of the system being related to the volume of the apparatus. Furthermore the fixation was complicated since the tambour had to be held by hand and so many recordings had to be discarded due to movement artifacts.

Hayashi et al. (1978) adapted the tambour by adding a thin rubber membrane with appropriate tension onto its surface. The device was fixed onto the anterior fontanelle with a piece of elastic band. The pressure, that is to say, the fluctuations in pressure were transmitted to an external transducer. They tested the apparatus in vitro, and also in vivo, measuring pressures in normal babies, and considered the apparatus to be reliable. However, the fixation of the device was very difficult requiring the absolute rest of the infant in order to obtain reliable recordings.

Another type of air filled tambour, based on a stethoscope was described by Blaauw et al. (1974). Recordings of more than one hour were performed, made possible by the use of a"small elastic strip for fixation. Dietze et al. (1972) modified a pneumatic arterial pulse pressure transducer for monitoring fontanelle pulsations. The authors were able to record different types 
of pulse waves, however quantitative pressure measurements were not possible, also they did not mention the fixation method.

\section{Methods based on the tension of the fontanelle}

As the methods described above were not suitable for reliable quantification of the fontanelle pressure other ways were sought to solve this problem.

Since the anterior fontanelle, consisting of skin plus underlying tissues could be compared to a membrane the comparison could be made with measuring intracavitary pressure. In order to measure intracavitary pressure through an intact and flexible membrane it is necessary that the bending forces of the membrane are eliminated or at least reduced. This principle had already been applied in obstetrics (Smyth 1957) and ophthalmology (Moses et al. 1962, Mackay et al. 1964, Quigley and Langham 1975). Originally intra-ocular pressure was measured by the Schiotz tonometer operating according to indentation tonometry, which means that for measuring pressure a fixed weight is applied and the depth to which the cornea sinks is observed. This method was considered inaccurate because of irregular forces and curvature in the corneal surface. Mackay (1964) considered aplanation tonometry superior to identation tonometry because with the latter method the forces required to flatten a given area of the cornea were also measured, while with the first method of flattening the cornea the tension forces were reduced to zero, so with any transducer intra-ocular pressure could be measured.

Just as in EDP measurements the principles for anterior fontanelle pressure measuring were derived from ophthalmology. Initially the identation tonometer, the Schiotz tonometer, was adapted for fontanelle pressure measurements (Davi-

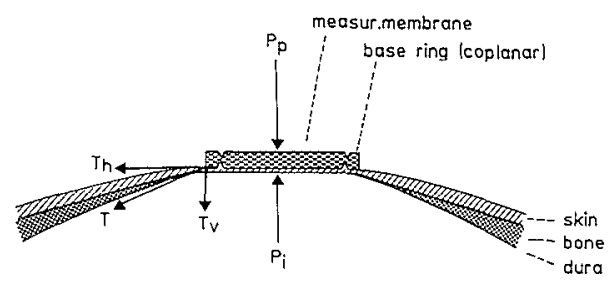

Fig. 6 Principle of aplanation tonometry on the fontanelle, regarded as a flexible membrane. The membrane tensions $(T=T h+T v)$ are eliminated by placing the base ring and so stretching the membrane. The pressure applied to the plunger or piston $(\mathrm{Pp})$ thus corresponds to the internal pressure $(\mathrm{Pi}) . \mathrm{Th}=\mathrm{T}$ horizontal; $\mathrm{Tv}$ $=\mathrm{T}$ vertical.

doff and Chamlin 1959). However this instrument was not considered reliable because the 'fontanelle membrane' does not have a constant elasticity like the corneal membrane. Furthermore unknown bending forces would interfere with the actual pressure monitoring. Besides the apparatus was handheld and had to be kept in an exact vertical position, perpendicular to the skin while the child was in a sitting position and had to be kept still. Therefore, this method was not considered practicle.

Efforts were made to develop a reliable fontanelle tonometer based on the aplanation principle, this being represented in figure 6 (Wealthall and Smallwood 1974, Edwards 1974, Salmon et al. 1977, Boston et al. 1976, Robinson et al. 1977, Daniels et al. 1981, Honda et al. 1982).

The devices were constructed with a base plate, to stretch the fontanelle, and with a central movable piston, plunger or rod. Any force transmitted across the fontanelle causes a relative displacement of this plunger which is converted into a pressure value. It was calibrated beforehand by establishing the correlation between standard pressures and standard weights ap- 
plied to the plunger.

It is important to mention that there is influence by gravitational forces. Therefore, to counterbalance these forces some authors made the piston of the tonometer spring loaded. In most reports attention was paid to the deformation of the scalp caused by the instrument load. Although with each new development that problem was 'practically' solved, application forces still influenced the actual pressure recording.

Another type of tonometer used in ophthalmology is the so called pneumo-tonometer or the pneumatic applanation tonometer, consisting of a sensing unit, a pneumatic tono-electric transducer, an amplifier and a recording unit (Durham 1965 et al., West et al. 1972, Quigley and Langham 1975). The device was used for measuring intraventricular pressure (Duggins et al. 1978) and also for fontanelle pressure (Menke et al. 1982, Easa et al. 1983). The basic mechanism consists of a continuous flow of gas through the membrane of the sensor tip which is restricted when the sensor is positioned. The restriction of gasflow through the sensor tip causes a rise in pressure within the central chamber. This is measured by a pressure transducer and processed by the recording unit providing a continuous, permanent recording on chart paper.

The problem with this tonometer type was the same as with the other types, i.e. it was handheld and therefore the application forces influenced the pressure recordings. The specific aspect of application pressure will be dealt with separately at the end of this chapter.

The pneumatic flow switch device (Numoto et al. 1966), described briefly on page 16 , served as a model for the development of the well known Ladd fiber optic device for measuring anterior fontanelle pressure. Other modifications of the pneumatic flow switch principle were presented as fontanelle pressure measuring devices (Whitelaw and Wright 1982, Kaiser and Whitelaw 1987a, Bunegin et al. 1987, Rochefort et al. 1987, Mehta et al. 1988). The authors all claimed reliability, although only a few authors performed comparative measurements between CSFP and AFP showing a good relationship between both pressures (Rochefort et al. 1987, Mehta et al. 1988).

The Ladd fiber optic device, derived from this flow switch, deserves a more detailed description here. The sensor contains a pressure sensitive membrane, three fiber optic columns (one input and two output fibers) and a pneumatic tube. A mirror is mounted on the membrane and employs a light source for sensing the position of the membrane which moves in response to internal pressure exerted on the dura (see figure 7).

Light transmitted through the input fiber strikes the mirror and is reflected back to the output fibers. When the mirror is

Fig. 7. Cross section of the Ladd fiber optic pressure sensor. $\mathrm{A}=$ input fiber, $\mathrm{B}=$ output fiber; arrows indicate the direction of light (see text).

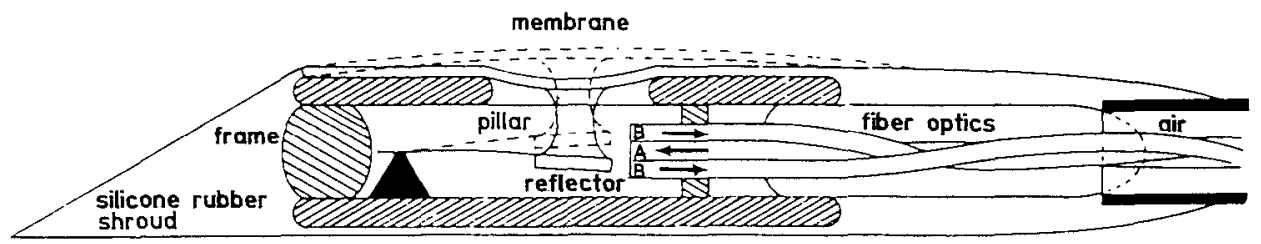


centered, each output fiber receives the same amount of light. Changes in pressure will move the mirror on the membrane off center so the output fibers will receive an unequal amount of light. The monitor contains a photo-electric detector which compares the amount of light carried by the two output fibers. The difference in light intensity then activates an air pressure bellows, which increases or decreases air pressure within the sensor. The instrument measures the air pressure transmitted to the sensor in order to restore the equilibrium of the mirror. This pressure, being equal to the intracranial pressure, is displayed on the monitor. Balancing occurs continuously following pressure changes and provides for automatic ICP or AFP monitoring.

Numerous studies concerning AFP monitoring on children with the Ladd fiber optic device were published (Levin 1977a, Vidyasagar and Raju 1977, Vidyasagar 1978, Vidyasagar et al. 1978, Philip 1979, Raju et al. 1980, Raju and Vidyasagar 1980, Von Wild and Pörksen 1980, Myerberg et al. 1980, Gaab et al. 1980, Philip et al. 1981, Hill and Volpe 1981a, Von Wild 1981, Von Wild et al. 1982, Bada 1983, McIntosh 1983, Walsh and Logan 1983). The studies dealt with AFP measurements on children of various ages and with various pediatric and neurological disorders. Both short term and continuous measurements were feasible.

Comparative measurements between CSFP and AFP were performed showing a good relationship (Myerberg et al. 1980, Ivan and Badejo 1983, Kaiser and Minnikus 1985).

The device could be easily calibrated. The response however was not very rapid and therefore rapid pressure fluctuations could not be detected. Application of the device consisted of simply holding it on the fontanelle or fixing it to the fontanelle with various fixation materials.

Transducers acting according to the principle of the Wheatstone bridge (e.g. strain gauge transducer) were also used as fontanelle pressure monitoring devices. When using such a transducer special attention was paid to proper co-planar application for which special holding devices were designed mentioned in the paragraph about fixation methods (Hirsch et al. 1978, Huch et al. 1979, Peabody et al. 1981, Gaab et al. 1982, Emery and Peabody 1983, Brömme et al. 1985, Kuramoto et al. 1986).

\section{Fixation methods}

Many authors paid attention to fixation procedures. Holbrook (1978) mentioned 'problems' but did not specify these. Philip (1979) and Donn and Philip (1978) used chin straps, after shaving the skin on and around the fontanelle. They felt that these straps could influence pressure measurements due to application pressure (after Horbar et al. 1980). Therefore they introduced an ECG electrode adhesive disk with good results. Plaster dressing was used by Von Wild and Pörksen (1980). A wide strip of soft sticking plaster over the device, which was placed in a rigid plastic disk, was used by Gaab et al. $(1980,1981)$ and Gaab (1984).

Foam pads (Hill and Volpe 1981a), specially developed holding devices (Horbar et al. 1980, 1981, Philip et al. 1981, Walsh and Logan 1983, Ivan and Badejo 1983), collodion (Walsh and Logan 1983, Kuramoto et al. 1986), plaster of Paris pads (McIntosh 1983) and rubber bands around the head (Huch et al. 1979, Brömme et al. 1985) have been described.

In the last 5 years more attention was paid to application forces when new holding devices were being developed. These hold- 
ing devices were tested on artificial fontanelles (Myerberg et al. 1980, Myerberg 1981, Walsh and Logan 1983), during comparative measurements between CSFP and AFP (Kaiser and Minnikus 1985) and also during continuous pressure monitoring (Gaab et al. 1980, 1981, Gaab 1984). Often the fixation did not hold for long due to the movements of the child, or to perspiration effects, resulting in unreliable pressure values.

The overall conclusion of the studies is that pressure devices with or without holders were applied to the fontanelle itself and not to the bony margins of the fontanelle so application pressure would interfere with ICP recording.

A special holding device, described in this thesis, was developed and tested, providing reliable AFP measurements without application pressure interfering with the actual pressure recording. This will be talked about in detail in chapter 3 .

\section{CONCLUSIONS}

1 VFP monitoring is the accepted standard method for ICP monitoring; other measuring techniques are compared with this technique.

2 In pediatric patients with closed sutures invasive ICP monitoring techniques have to be used. Of all techniques described, telemetric epidural pressure recording with a transducer properly applied to the dura has the least disadvantages. However, in interpreting measurement results one should know that EDP tends to be slightly higher than VFP due to mechanical characteristics of the dura.

3 In children with an open fontanelle AFP monitoring is usually superior to invasive methods, however, only if proper attention is paid to the measuring device itself and to its application on the fontanelle.
4 The most frequently used transducers for fontanelle pressure monitoring are the Ladd fiber optic device, modifications of the pneumatic flow switch, and several types of strain gauge transducers.

5 The aplanation principle is by far the best for measuring fontanelle pressure, comparable with measuring intracavitary pressure through a flexible membrane.

6 Application and fixation methods for AFP monitoring have not been standardized so far. Therefore, the results of all authors have to be interpreted according to the fixation method used with respect to interfering application forces.

7 The ideal fixation method should overcome the influence of application forces on the non-invasive measurement of ICP via the anterior fontanelle. 


\section{THE ROTTERDAM TELETRANSDUGER}

\section{INTRODUCTION}

After reviewing the various ICP monitoring techniques, it is clear that, as far as invasive methods are concerned, besides other disadvantages the infection risk is the major factor limiting the time-period over which ICP can be measured. Telemetric epidural measurement has several advantages. There is the possibility of long term monitoring without increased infection rate. Intermittent measurements over extended periods of time, even in the outpatient clinic, are feasible. The procedure is less invasive and no risk of damage to cerebral tissue exists.

In the neurosurgical department of the University Hospital of Rotterdam, in col- laboration with the Central Research Workshop of the Erasmus University of Rotterdam, a passive implantable device for epidural telemetric ICP monitoring in patients with closed sutures was developed by $\mathrm{DA}$ de Jong. The principles and the specific technical details of the implantable device have been extensively described by De Jong et al. (1975, 1979, 1982, 1983). In this chapter first the technical details and the implantation technique will be briefly summarized whereafter in a separate section the details of measuring anterior fontanelle pressure with the RTT in newborn children and infants will be dealt with.

\section{DESCRIPTION OF THE DEVICE AND ITS APPLICATIONS}

\section{Technical details}

The system consists of:

- The batteryless operated Rotterdam Teletransducer (figure 1).

- A bedside monitor or detection unit; basically a calibrated high frequency (HF) sweep generator (figure 2).

- A measuring probe with an incorporated antenna coil for conducting the HF radiowaves to the transducer (figure 3 ).

The RTT consists of a pressure sensitive capacitor in parallel with an inductor coil for receiving the radiowaves emitted by the monitor's antenna coil (figure 2).

The resonance frequency $(\mathrm{Fr})$ of the transducer falls when rising pressure $(P)$ is exerted on one of the capacitor plates. A linear relationship between $\mathrm{dFr}$ and $\mathrm{P}$ was obtained. The capacitor is formed by a fixed silver plate in parallel with a titanium membrane. This pressure sensing membrane is applied co-planar with the dura. Alterations in (epidural) pressure will induce changes in the plate distance of the capacitor thereby altering the capacity, and thus the resonance frequency of the transducer.

The external detection unit consists of an oscillator, continuously sweeping over a narrow band of frequencies $(95 \pm 10 \mathrm{MHz}$, $200 \mathrm{~s}^{-1}$ ) and a calibrator circuit. This sweep frequency oscillator energizes the capacitor circuit through induction. Pressure fluctuations will cause changes in resonance frequency $(\mathrm{Fr})$, which are con- 


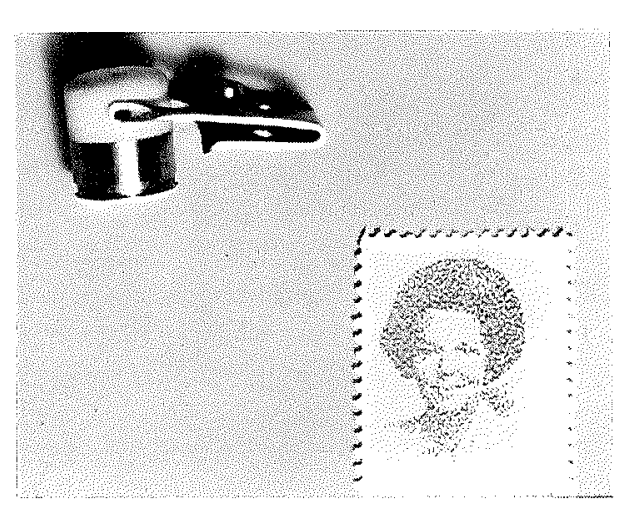

Fig. 1 The Rotterdam Teletransducer shown next to a regular Dutch stamp.

verted into a pressure analogous voltage through the built in electromagnetical impedance measuring device (transformer principle). The contact between the transducer and the detection unit is established by radiowaves (figure 3). Through these technical characteristics the RTT can record the rapid pulsations induced by cardiac output and respiration.

In Table I the technical details of both the RTT and the bedside monitor are given.

In figure 4 the transducer (glass bond type) is shown in detail.

The pressure sensing membrane consists of a titanium diaphragm of $20 \mu \mathrm{m}$ thickness and a fixed silver plate at $50 \mu \mathrm{m}$ distance. The titanium diaphragm is bonded to a titanium ring by laser welding, thus achieving a hermetic seal. Titanium, being biocompatible and inert to body fluids, was chosen for its favourable bonding abilities to the material itself and to ceramics. Non porous ceramics were chosen for encapsuling the coil capacitor. These ceramics are transparent to radiowaves, biocompatible and impermeable to water.

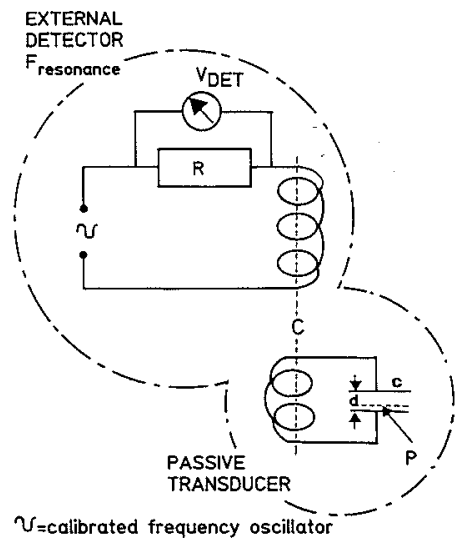

Fig. 2 Schematic representation of the RTT and the detection unit. The system acts as an L-C-circuit i.e. a pressure sensitive capacitor (c) in parallel with an inductor coil receiving the radio waves emitted by the monitor's antenna coil.

$\begin{array}{ll}\mathrm{F}_{\mathrm{r}} & =\text { resonance frequency } \\ \mathrm{d} & =\text { distance } \\ \mathrm{P} & =\text { pressure } \\ \mathrm{V}_{\text {det }} & =\text { voltage detection } \\ \mathrm{R} & =\text { resistance } \\ \mathrm{c} & =\text { capacitor } \\ \mathrm{C} & =\text { inductive coupling }\end{array}$

Barometric pressure changes are automatically compensated for.

Changes of the bodytemperature of the patient can be entered manually or can be automatically compensated for via an input from the patients temperature sensor.

Once the device is implanted in adult patients no facilities exist for recalibrating. Diffusion of water into the transducer would result in unpredictable zero drifting due to changes in the vapour pressure within the transducer housing. This requires hermetic sealing between the titanium pressure sensing part and the ceramic housing of the transducer. Therefore, special attention was paid to bonding 


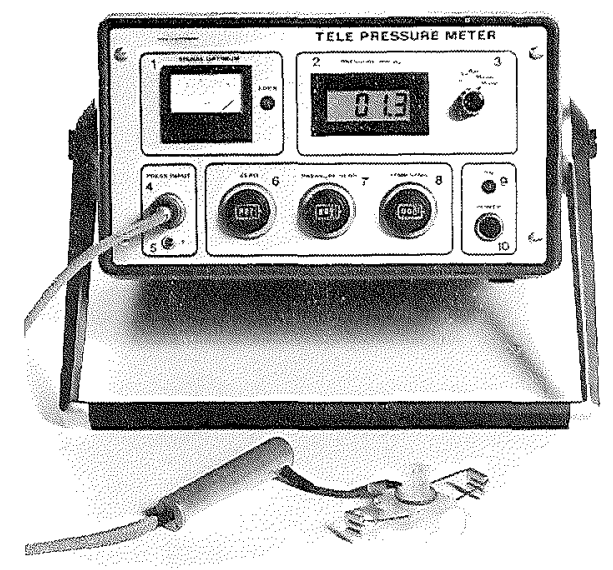

Fig. 3. Frontside of the bedside monitor or detection unit, including the connected measuring probe and the fontanelle adaptor.

1. Scale indicating relative position of the pressure sensor $(0-500 \mathrm{mV})$, with the lamp indicator (Lock) for contact.

2. Digital pressure display (0-100 $\mathrm{mmHg}$ ).

3. Dial for calibrating and measuring.

4. Input for measuring probe.

5. Mode for chosing epidural (E) or fontanelle pressure (F). In EDP-mode auto-compensation of simultaneously measured barometric pressure is built in. Dimensions (lenght $X$ width $X$ height): $30 \times 25 \times 15 \mathrm{~cm}$.

6. Dial adjust for zero.

7. Dial adjust for pressure sensitivity.

8. Dial adjust for temperature sensitivity only in EDP.

9. Lamp indicator for power on.

10. Power on-off switch.

techniques. Epoxy resin, active metal brazing and glass bonding techniques were investigated. In the end bonding with epoxy resin turned out to be the best for watertight sealing of the transducer.

\section{Implantation of the trans-}

\section{ducer}

For the purpose of implantation both in

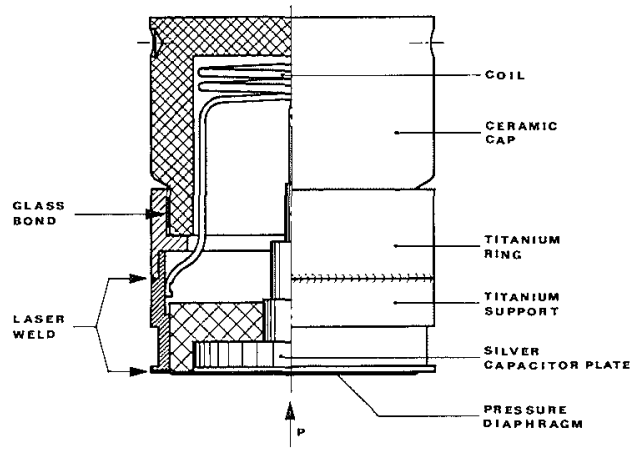

Fig. 4. Schematic representation of the Rotterdam Teletransducer (glassbond type).

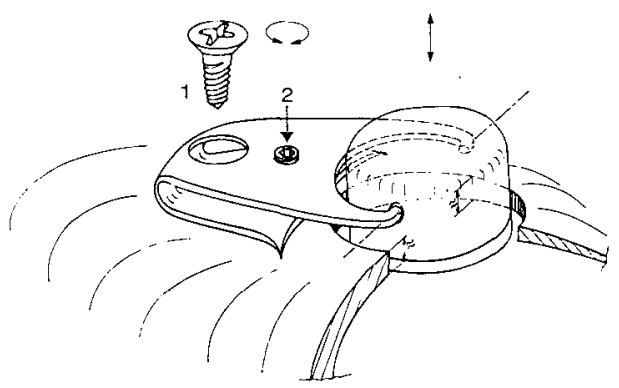

Fig. 5. Transducer mounting clip; 1 fixation screw, 2 calibrator screw for varying the insertion depth.

animals and in humans the RTT was mounted in a stainless steel clip, allowing regulation of insertion depth by a small calibrator screw (figure 5). Depth positioning was performed visually, while observing contact with the dura, or it was performed according to the pressure depth curve.

The in vivo experiments performed with the transducers were concentrated on the evaluation of the pressure response in an animal model and on the transducer's stability during the longer implantation periods.

Later on, after accomplishing adequate watertight sealing, the RTT was implanted in twenty-two adult patients with various disorders, most with severe head 
TABLE I: Technical details of the RTT and the bedside monitor.

$\begin{array}{ll}\text { Specifications of the RTT } \\ \text { Materials } & : \text { titanium and macor } \\ \text { Dimensions: diameter } & : 10 \mathrm{~mm} \\ & : 9-13 \mathrm{~mm} \\ \text { Weight height } & : 2 \text { gram }\end{array}$

Sterilization method : gas: ethyleen dioxide $\left(52^{\circ} \mathrm{C}\right)$

Temperature sensitivity : $-2 \mathrm{mmHg} /{ }^{\circ} \mathrm{C}$

Conditions in vitro $: \pm 2 \mathrm{mmHg}$

(saline $37^{\circ} \mathrm{C}$ )

(zerodrift, repeatibility

and hysteresis)

Pressure range $\quad:-50-+100 \mathrm{mmHg}$

Estimated diaphragm $\quad: 0.2 \mu \mathrm{m} / 10 \mathrm{mmHg}$

deflection

Frequency response $\quad:>50 \mathrm{~Hz}$

Method of application : co-planar depth positioning

Characteristics of the bedside monitor

Supply : $220 \mathrm{~V}-\mathrm{AC}$

Rated power

consumption

: $20 \mathrm{~W}$

Output specification

$\begin{array}{ll}\text { HF sweep } & : 85-105 \mathrm{mHz} \\ \text { Sweeptime } & : 5 \mathrm{~ms}(200 \mathrm{~Hz}) \\ \text { HF-output voltage } & : 500 \mathrm{mV}\end{array}$

injury, all considered at risk from raised intracranial pressure. In eleven patients the RT'T was removed after one week, in the other patients the device remained in situ. In this way long term ICP monitoring was possible not only for as long as the patient remained in hospital but also after discharge, in the out-patient clinic.

After these first experiences the implantation procedure was improved and the need for accurate depth setting ana- lyzed. In the initial implantations in patients depth setting of the transducers on the dura was performed visually without performing a pressure depth curve. However, in two patients an unrealistically high EDP level was obtained. Thereafter the transducer was repositioned accurately according to the pressure depth curve. This is shown in figure 6. After this experience the transducer was always positioned according to the pressure depth curve.

Occasionally the dura was damaged when making a burrhole, but this did not influence the pressure recordings. In ten patients VFP was measured simultaneously. Regression analysis averaged over these 10 patients resulted in: $\mathrm{EDP}=1.09 \mathrm{VFP}$ - $1.0 \mathrm{mmHg}$ (Maas and De Jong 1986a,b). Up to this date the RTT has been routinely and successfully used for continuous ICP recording in patients with normal pressure hydrocephalus.

Implantable transducers are of the closed type. The response includes variations in barometric pressure as well as the effect of ambient temperature. Physiological pressure values are always relative to barometric pressure, therefore the monitor incorporates a separate barometric pressure sensor and an entry for body temperature for auto-compensation of the pressure values.

The open type RTT was used for fontanelle pressure monitoring. This type is not automatically compensated for bodytemperature and barometric pressure, because the response in AFP measurements is not dependent on variation in barometric pressure and environmental temperature. 


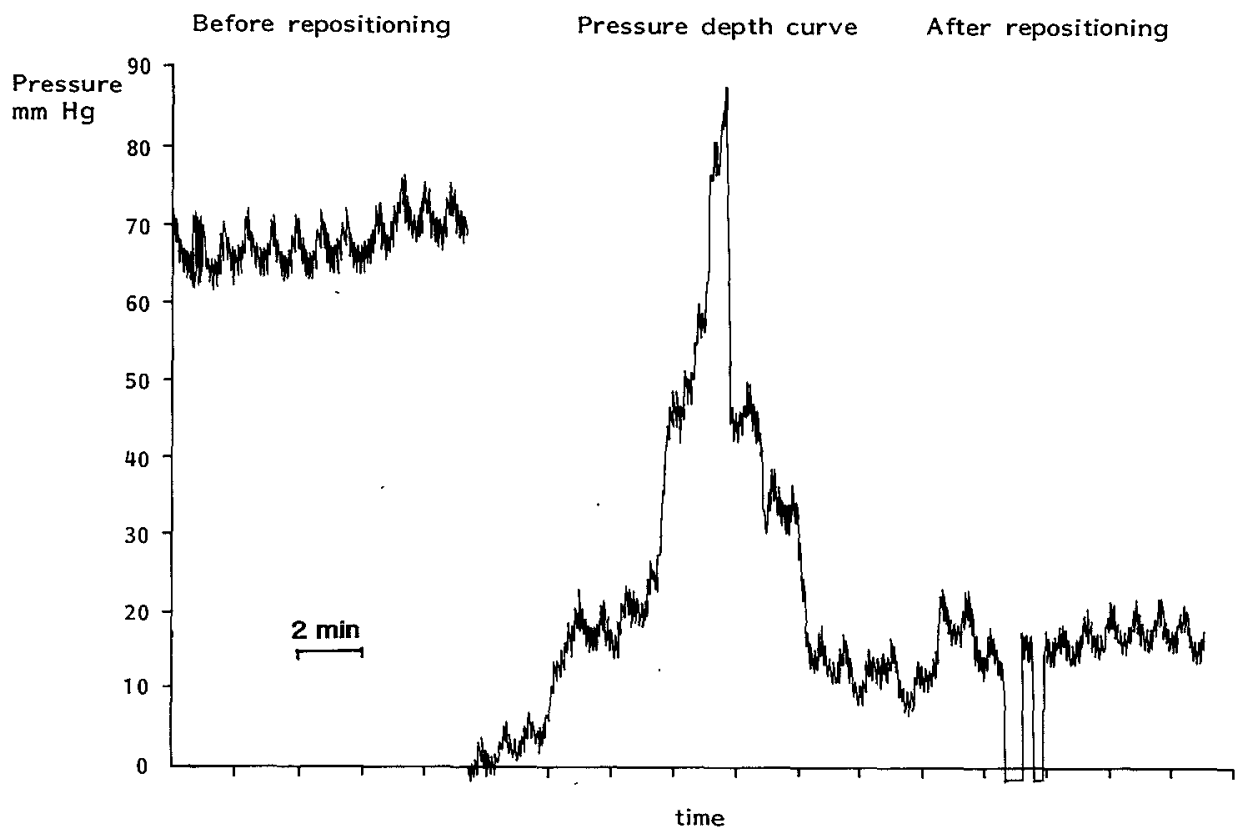

Fig. 6. Pressure depth curve and EDP recording before and after repositioning of the RTT on the dura (Reproduced, with permission, from Maas and De Jong 1986, Acta Neurochir 79:512).

Anterior fontanelle pressure monitoring with the Rotterdam Teletransducer

A natural access to the intracranial compartment in newborns and infants is given by the patent anterior fontanelle. Noninvasive monitoring of $\mathrm{ICP}$ by means of the fontanelle has already been frequently attempted (see chapter 2). In analyzing the problems encountered by others and in observing the aspects of the fontanelle we noticed the similarity between epidural and fontanelle pressure measurements, the main difference being the skin (including underlying subcutaneous tissue) interposed between the dura and the transducer. The major problem in AFP measurements is the application pressure. The concept of controlled application pressure (Brömme et al. 1985) was changed for that of controlled depth setting in order to compensate for the tension in any of the underlying tissues. A special light weight perspex skull applicator was designed. This so called 'adaptor' weighs 10 gram and has facilities for accurate depth setting. In figure 7 the adaptor, containing the RTT, is shown.

The transducer is fitted in a perspex piston which can be moved up and down in the adaptor which in its turn is securely maintained on the skin overlying the bone adjacent to the fontanelle.

Ideally the natural movements of the child should not interfere with the AFP measurements. Therefore a fixation frame was constructed for keeping the adaptor and the RTT accurately in position on the child's head. This light weight frame consists of a loading spring connected to soft silicone tubing around the chin and occiput of the child. This tubing is joined 


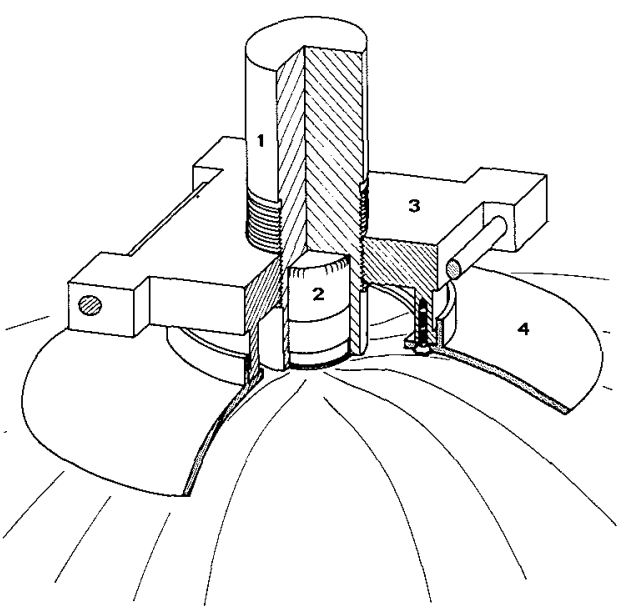

Fig. 7. Cross section through the special skull adaptor, containing the Rotterdam Teletransducer, for anterior fontanelle pressure measurements. 1: perspex piston containing transducer; 2: transducer; 3: perspex adaptor; 4: adaptor plate fixing device to the skin, overlying the bony surroundings of the fontanelle.

together by $\mathrm{T}$-shaped connectors which are located directly above the ears. In figure 8 the fixation frame and the adaptor are shown.

This system prevents axial displacement and accommodates for the necessary application force. The measuring probe is loosely connected to the adaptor by means of a flexible wire not interfering with the movements of the child's head. This measuring probe consists of a sealed perspex or anodized aluminum enclosure for the electrically insulated antenna coil used for transmitting the HF-radio waves to the passive pressure sensor.

Preferably, for proper fixation the skin at and around the area of the fontanelle is shaved. In figure 9 the adaptor with the RT'T is shown, fixed on the head of a child by means of the fixation frame.

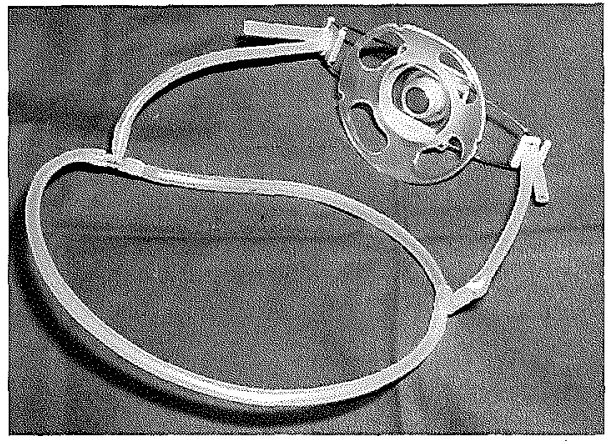

Fig. 8. Fixation frame, consisting of soft silicone tubing, interconnected by $\mathrm{T}$-shaped, connectors, and a loading spring attached to the skull adaptor containing the RTT.

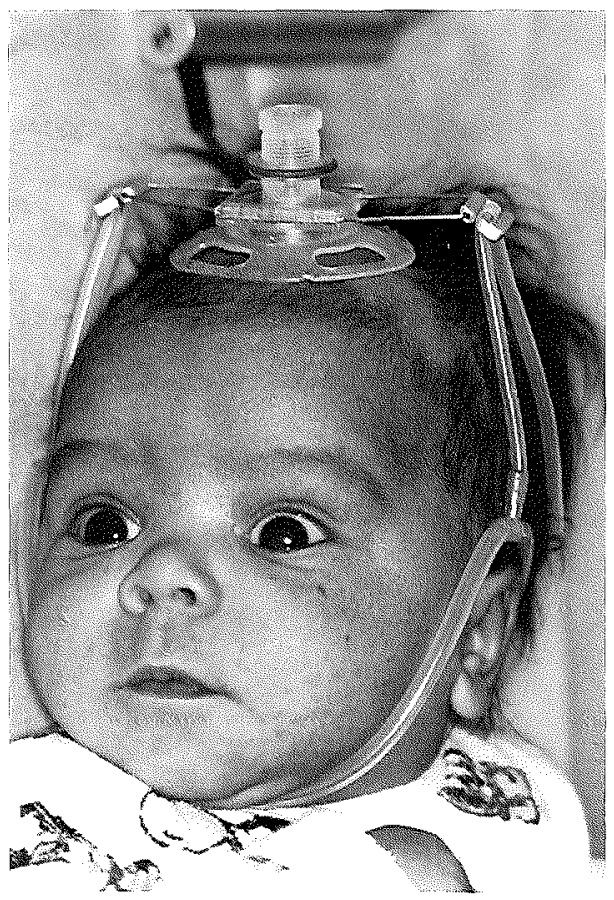

Fig. 9. Skull adaptor containing the RTT, positioned on a child's head and kept in place by means of the fixation frame.

If necessary, adhesive tape is placed on part of the adaptor plate and the skin, thus preventing lateral dislocation. This is shown in figure 10. 


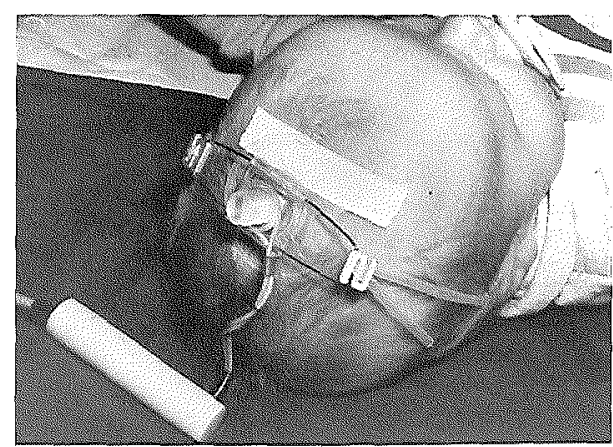

Fig. 10. Skull adaptor on a child's head. Lateral dislocation is prevented by using adhesive tape.

Figure 11 shows that the child is able to move around freely while AFP is being measured. The connection between the transducer and the bedside monitor by means of the flexible wire of the measuring probe is clearly shown.

The complete system has been thoroughly tested for electrical safety and is considered safe to use in patients.

\section{Technique of application and depth setting in AFP monitoring with the RTT}

As in epidural pressure measurement accurate depth positioning of the transducer in AFP monitoring is essential. Proper depth setting of the transducer is performed manually by uniformly rotating the piston in its thread (figure 12).

Insertion depth is indicated by an incorporated calibrated mechanical dial. Contact between the transducer and the detection unit is established by radio waves. The extent to which electromagnetic energy is transferred from the detector coil is a relative measure for the position of the transducer and this is recorded on the same chart. In this way manual artifacts can be excluded (figure 13).

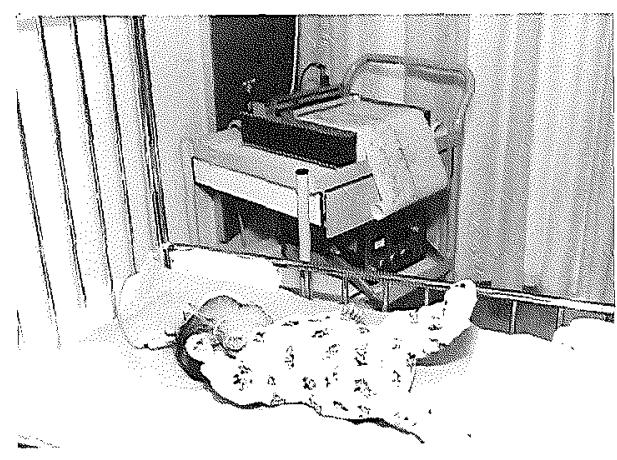

Fig. 11. The skull adaptor and the fixation frame on a child's head. In this figure the child's ability to move around freely is shown.

\section{The pressure depth curve} in AFP monitoring

The adaptor containing the RTT is mounted on the skin surrounding the fontanelle by means of the silicone tubing of which the open ends are connected to the lever of the adaptor. The silicone tubing is slightly stressed to maintain the adaptor just on the head.

The calibration procedures for 0 and 40 $\mathrm{mmHg}$ are performed.

Manual rotation of the piston leads to depth setting of the transducer. Just prior to the point at which the transducer touches the skin and no pulsations are seen, zero set point for both the pressure and the insertion depth are established on the chart using the special dials of the monitor (figure 3). By uniformly rotating the piston the insertion depth of the transducer is gradually increased and a characteristic PDC appears on the chart (figure 13). Initially the pressure rises until a plateau $(\mathrm{Pa})$ is reached at which cardiac pulsations remain constant.

On further insertion the transducer traverses the subarachnoid space and does not encounter extra opposing forces and therefore a further rise of pressure is delayed. The plateau pressure thus recorded is 


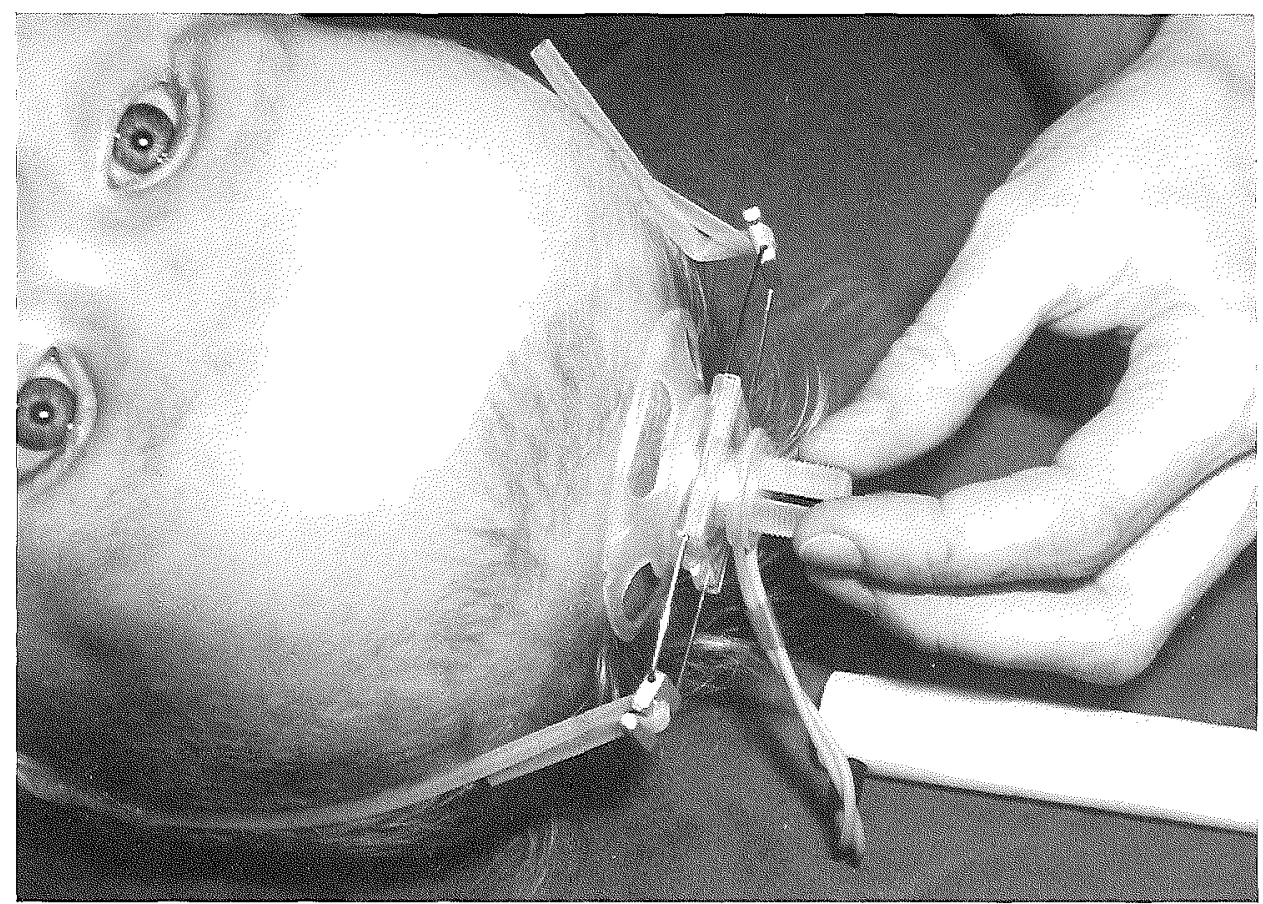

Fig. 12. Skull adaptor, containing the RTT, on a child's head. Depth setting of the transducer is performed by manually and uniformly rotating the piston in its thread.

equal to ICP. On further increasing the depth of the transducer a sharp rise in pressure is observed, due to compression of the subarachnoid space and brain tissue. On subsequent withdrawal of the transducer the PDC is recorded in reverse, reaching the plateau pressure $(\mathrm{Pb})$ and eventually zero, as the transducer is again withdrawn to a point just beyond contact with the skin (Plandsoen et al. 1986a,b). When the RTT is applied repeatedly, subsequent identical pressure recordings can be obtained. The procedure is simple and can be performed by any serious investigator.

The PDC's resemble those described by Schettini and Walsh (1974) and obtained in epidural pressure measurements. This lends support to the idea that the pressure measured at the 'plateau' represents actual
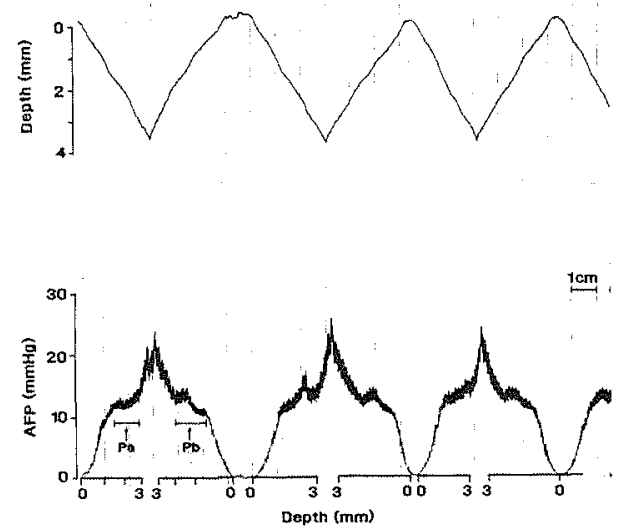

Fig. 13. Subsequently performed characteristic pressure depth curves in a short term AFP measurement on a newborn infant. Insertion depth is shown at the top margin. $\mathrm{Pa}$ represents the plateau reached on insertion of the $\mathrm{RTT}, \mathrm{Pb}$ represents the plateau reached at withdrawal of the RTT. 
ICP.

The level of ICP can be determined from the PDC according to the method described in chapter 4 .

The AFP measurements consisting of. PDC's only will be called short term measurements.

For long term or continuous AFP monitoring the transducer is set at the depth of the plateau of the pressure depth curve. With the fixation frame the transducer is maintained in place constantly.

\section{AFP monitoring and application pressure}

In recording epidural pressure measurements it is important that the transducer is kept in place (at accurate depth) according to the co-planarity principle. In AFP monitoring with the RTT this is also the main aspect. To this end the special fixation frame already mentioned was designed for maintaining the transducer at accurate depth in the fontanelle. Many fixation procedures tend to interfere with the exact recording of ICP due to unexpected rises in application force. The mechanical principle of application forces related to anterior fontanelle pressure monitoring was studied and this eventually resulted in the skull adaptor with the fixation frame, already described.

The underlying principle is the so called 'balance of forces'. All forces exerted on a surface have natural reaction forces. If for example a table rests on a floor it does not fall through due to the natural reaction forces. See figure 14 for explanation. If any weight is placed on the table it still does not fall through, due to the constantly balanced forces. This balance is dynamic and constantly changing according to the exerted pressures.

As long as the position of the transducer is maintained, the pressure to be measured
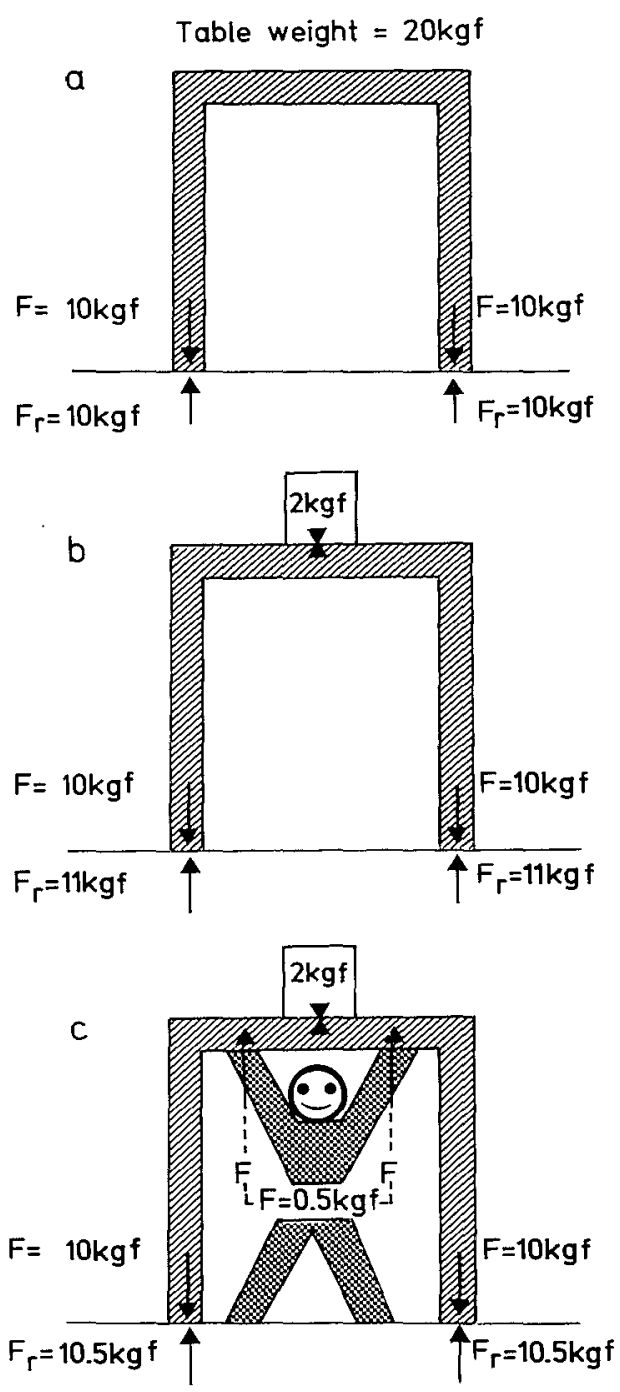

Fig. 14 a, b, c Concept of naturally acting reaction forces $\left(\mathrm{F}_{\mathrm{r}}\right)$ constantly changing according to the balance of forces. a) A table of 20 kgf exerts a force (F) of $2 \times 10 \mathrm{kgf}$ on the floor. b) If $2 \mathrm{kgf}$ is added, the force on the floor increases, so do the reaction forces. c) When a person tries to raise the table, the reaction forces are reduced in proportion to the exerted force. 


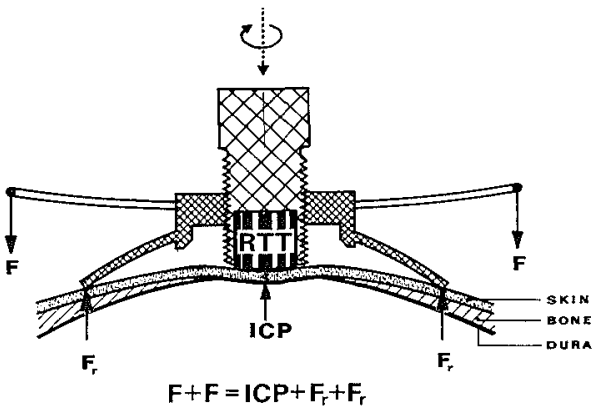

Fig. 15. Principle of measuring ICP on the fontanelle with the Rotterdam Teletransducer in the light weight skull adaptor. Note the balance of forces: forces $(F)$ for both aplanation and application do not interfere with ICP monitoring as surplus of these forces runs off to the skull by means of the special adaptor and fixation frame. $\left(\mathrm{F}_{\mathrm{r}}=\right.$ reaction force). and exerted on the diaphragm is continuously balanced by naturally generated reaction forces. Surplus application forces run off to the skull. As a result of this method application pressure does not interfere with the recording of ICP. In figure 15 the balance of forces in the case of the skull adaptor is shown.

This application method and its mechanical basis has not been described by others. Authors reporting so far on fontanelle pressure measurements (chapter 2) have either had problems with proper fixation or they did not describe exactly how the device had been applied to the fontanelle. Therefore the reported differences in pressure values might be due to different application techniques, since, generally speaking, fixation methods may interfere with the recording of ICP.

\section{CONCLUSIONS}

1 The fully implantable batteryless Rotterdam Teletransducer has proven to be a reliable device for measuring epidural pressure in patients with closed sutures. Its main technical features are: the good frequency response, the zero stability, the dynamic performance and the co-planar setting which reduces dura tensions to zero.

2 The EDP co-planarity principle is valid for measuring ICP non-invasively via the anterior fontanelle, since in children with an open fontanelle only the skin with subcutaneous tissue is overlying the dura, the critical matter being only that the transducer has to be maintained at accurate depth in the fontanelle. Furthermore, application forces should not interfere with the recording of ICP. To meet this end, a special light weight skull adaptor, containing the RTT, was designed. The adaptor plate rests on the skin overlying the bone adjacent to the fontanelle. A light weight frame, consisting of a loading spring and soft silicone tubing, maintains the skull adaptor in place. The system prevents axial displacement and accommodates for the necessary application force. 3 According to the balance of forces the application forces do not interfere with the recording of ICP as long as the transducer is kept at accurate depth in the fontanelle. The accurate depth of the transducer is determined by performing the so called pressure depth curve. 


\section{H A P T E R 4}

\section{ANTERIOR FONTANELLE PRESSURE IN NORMAL NEWBORNS AND INFANTS}

\section{INTRODUCTION}

Every new measuring technique for use in human beings requires its own 'normal values'. These values should be obtained in persons not suffering from any disease at the moment of the measurement (Wulff 1981).

For the purpose of establishing normal values of AFP with the RTT in the fontanelle adaptor it was necessary to investigate a number of normal children. Furthermore a review of the literature on normal values of AFP obtained with other methods, was necessary to find out whether the AFP values obtained with our method were in agreement with the values reported in the literature.

Also the question arose as to whether normal levels of AFP would be different from normal ICP levels in children, measured with invasive methods.

Several authors reported a positive relationship between ICP and age (Vidyasagar and Raju 1977, Hayashi 1978, Welch 1978, 1980, Philip et al. 1981, Lopez Ibor et al. 1982). This relationship was found with both invasive and non-invasive measuring techniques (Robinson et al. 1977, Peabody et al. 1981, Paraicz 1982, Easa et al. 1983). Therefore in selecting the children we chose newborns and infants of various ages to find out whether with this method the same age/pressure relationship could be established.

It is obvious that in older children monitoring of ICP is more difficult than in younger children, mainly due to their restlessness.

During the AFP measurements we experienced difficulties performing pressure depth curves in fontanelles of smaller size. This sometimes resulted in bad quality of the recordings.

The question arose as to whether the size of the fontanelle was important in AFP monitoring and it was decided to measure the fontanelle size according to a method reported by Popich and Smith (1972). With this simple method the mean fontanelle size is calculated from the length and the width of the fontanelle. Only in children with a fontanelle of $1 \mathrm{~cm}$ diameter or more could we measure AFP, since the diameter of the RTT measures $1 \mathrm{~cm}$.

The aims of this part of the study were - To record AFP in normal newborns and infants and to estimate its normal range. - To study the relation between AFP and age of newborns and infants.

- To establish values of fontanelle size in these normal newborns and infants and to compare them with those found by Popich and Smith.

- To study whether the fontanelle size was important in measuring AFP.

- To investigate whether in the literature normal ICP values in children measured with non-invasive methods differ from ICP values measured with invasive methods. 


\section{MATERIAL AND \\ METHODS}

A checklist was developed to exclude those babies who suffered from pre-, peri-, and postnatal detrimental influences, with $\mathrm{em}$ phasis on those factors influencing the brain and the CSF circulation. As is known from the literature premature born babies carry a great risk of developing intraventricular haemorrhage which may cause a rise in ICP (Haase 1986, Volpe 1987, 1989a,b). Diseases of the mother during pregnancy, e.g. infections due to cytomegalovirus or toxoplasma and use of medication, may cause damage to the developing embryo (Brett 1983, Lindhout 1985). When during labour a forceps has to be used the skull and the brain of the baby may be injured. A long period of asphyxia during or after birth may also influence ICP (Donn and Philip 1978, Levene et al. 1987). Certain metabolic diseases or chronic diseases (such as cardiac disease), diagnosed after birth, cause damage to the developing brain and may influence ICP (Adams and Lyon 1982). Therefore, before a child was selected as normal and 'measurable' it had to meet the following criteria.

- No diseases of the mother during pregnancy

- Duration of pregnancy above 36 weeks

- No complications during labour

- Apgar score above 8 after one minute

- Birth weight above 2500 gram

- Normal psychomotor development of the baby up until the time of the AFP measurement

- No known neurological or pediatric disease

- Size of the fontanelle $1 \mathrm{~cm}$ diameter or more.

Eighty two infants were studied and divided into two groups.
Group I consisted of 30 newborn babies (12 males, 18 females) aged 0-9 days. The age distribution, in classes of 2 days, is shown in figure 1 . These babies were born in the obstetrical ward of the University Hospital of Rotterdam. Informed consent of the mothers was obtained before the AFP recording. The measurements were performed from September 1985 until November 1986.

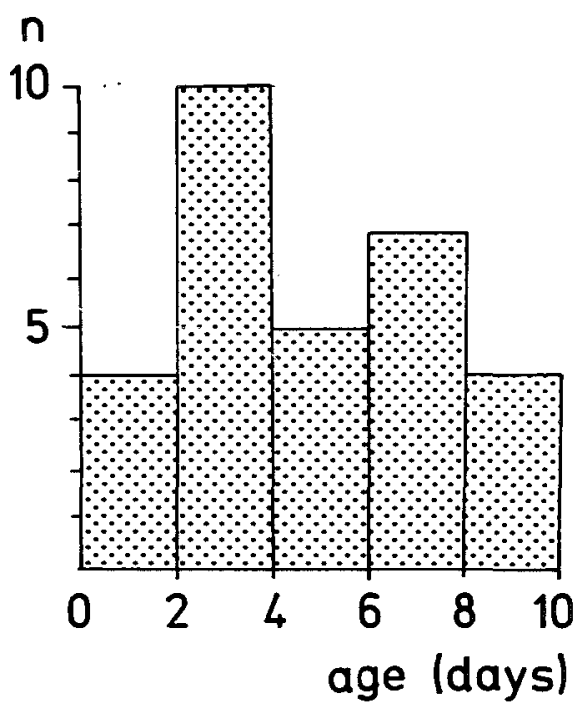

Fig. 1 Age distribution, in classes of 2 days, in group $I$ (30 newborns). ( $\mathrm{n}=$ number of newborns).

Group II consisted of 52 infants (28 males, 24 females) aged 30-330 days. The age distribution, in classes of 60 days, is shown in figure 2. These infants were measured in the child health clinic in the 'Zevenkamp' district of Rotterdam (one of the new suburbs with large numbers of young children). All children in the Netherlands are seen at regular intervals 


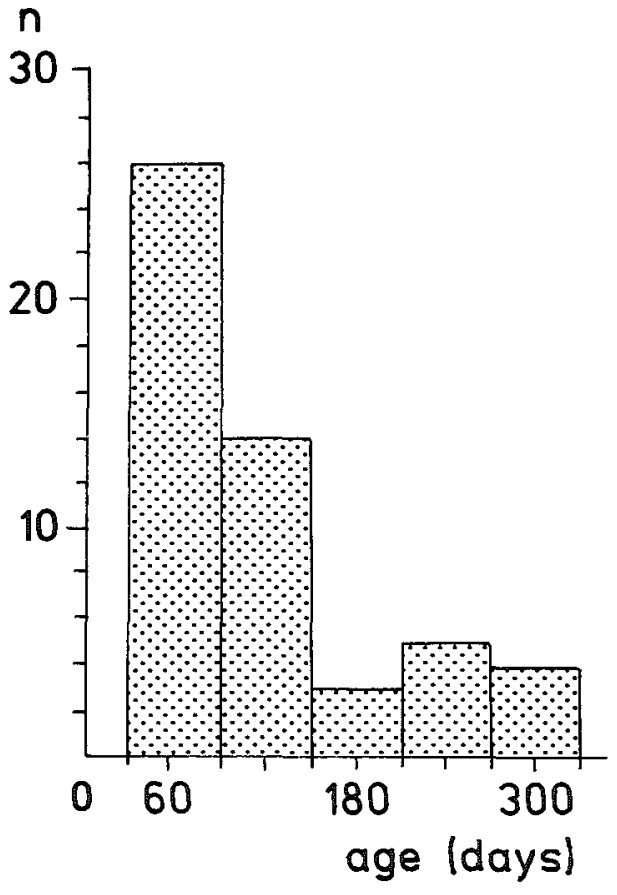

Fig. 2 Age distribution, in classes of 60 days, in group II (52 infants). ( $\mathrm{n}=$ number of infants).

in pre-school child health services distributed over various urban districts. The child health maintenance is organized by the Cross-organization which also provides home nursing care. The study population of children in the Zevenkamp district was considered representative for the total population of children in the Netherlands.

The medical doctor in the child health clinic asked a total of 128 mothers whose babies met the above mentioned selection criteria, to co-operate with their babies in the so called 'Fontanelle Project'. Before giving informed consent the mothers were given a simple written explanation of the project and of the measurement procedure. Reasons for not giving informed consent were of personal nature. The measurements were performed from January 1987 until February 1988.

\section{AFP recordings}

One of the requirements of a successful AFP recording is a quiet baby. In group I the babies were asleep practically all the time. In group II we scheduled one hour to enable us to quieten the baby (and its mother).. Giving a small feed was usually successful. In neither group I or group II were the children sedated with drugs. During the AFP recordings the babies and the infants were in the supine position without elevation of the head.

For the AFP recording an analogue signal recorder was used (W\&W, model 314).

The technique for measuring AFP was described in chapter 3. During each AFP recording a number of at least three pressure depth curves (PDC's) was performed. Figure 3 shows a part of an AFP recording performed in a newborn baby of group I. The ascending plateau is called $\mathrm{Pa}$, the descending plateau $\mathrm{Pb}$.

\section{AFP calculation}

The AFP value of a single PDC was calculated as the average of the ascending plateau $(\mathrm{Pa})$ and the descending plateau $(\mathrm{Pb})$. The calculation of AFP from the recordings was done by means of a specially developed perspex 'ICP calculator', displayed in figure 4 . The scale of the small inner bar represents half the scale of the large outer bar. The scale accords with that of the paper of the signal recorder.

For calculating the AFP from a recording with the ICP calculator the small inner bar is first placed on a plateau of a pressure depth curve. The amplitude of the pulsations is measured, in this case $1 \mathrm{mmHg}$. This is the equivalent of twice the true amplitude $(2 \mathrm{mmHg})$. Thereafter the large outer bar is moved along the small bar until the zero of the small bar is opposite the equivalent of the measured amplitude value of $1 \mathrm{mmHg}$. The AFP value in this 

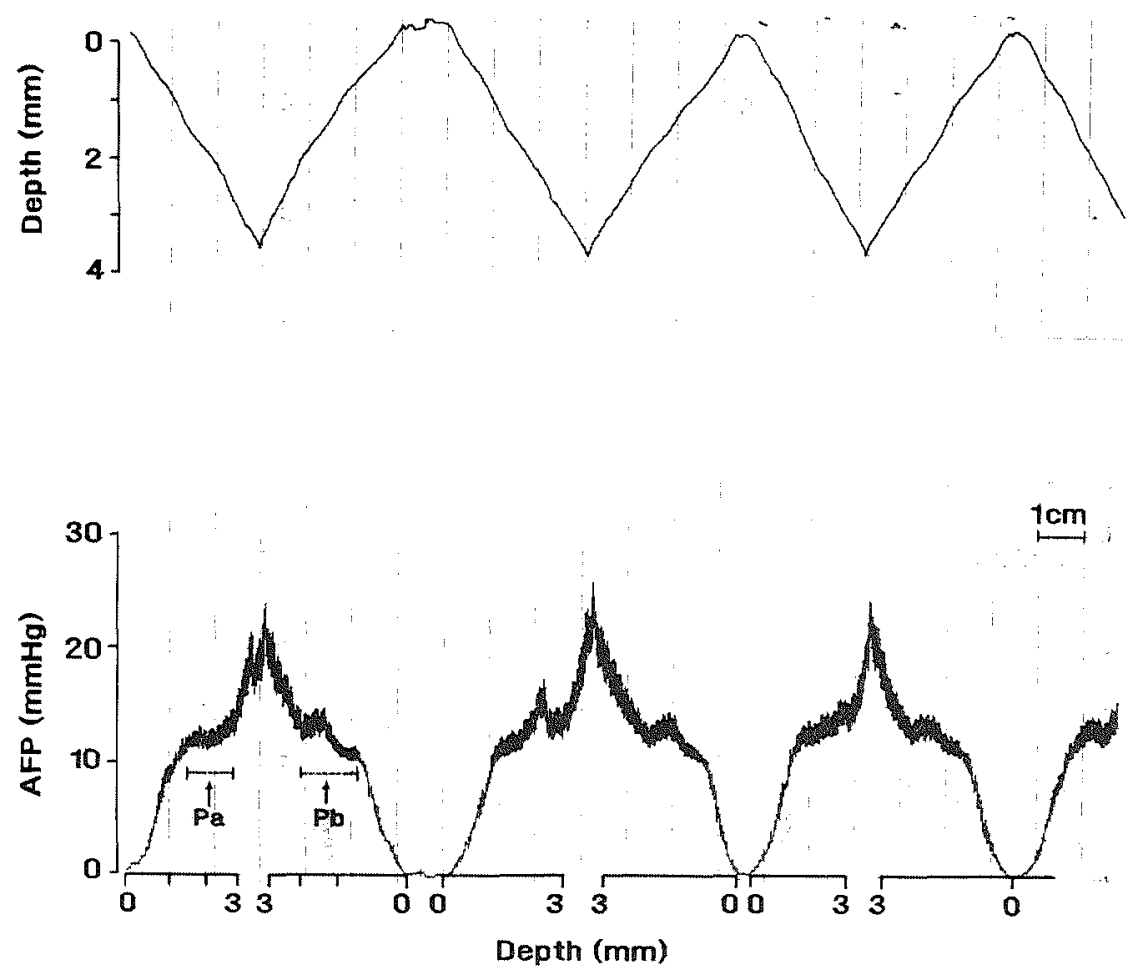

Fig. 3 Part of an AFP recording in a healthy newborn baby of group I, showing three consecutive pressure depth curves (PDC's). $\mathrm{Pa}=$ ascending plateau of the $\mathrm{PDC}, \mathrm{Pb}=$ descending plateau of the PDC.

Fig. 4 ICP calculator. The large outer bar and the small inner bar are shown separately. The scale is the same as that on paper of the signal recorder. The scale of the small inner bar is half the scale of the large outer bar. PP (= pulse pressure) on this scale is presented as the average of two pulsation frequencies: a rapid oscillation corresponding to arterial pulsation and a slower waveform synchronous with respiration (Miller 1978).

recording was $11.5 \mathrm{mmHg}$. This is the point at the left side of the large bar where the bar crosses the horizontal axis of the recording. In figure 5 the procedure is shown.

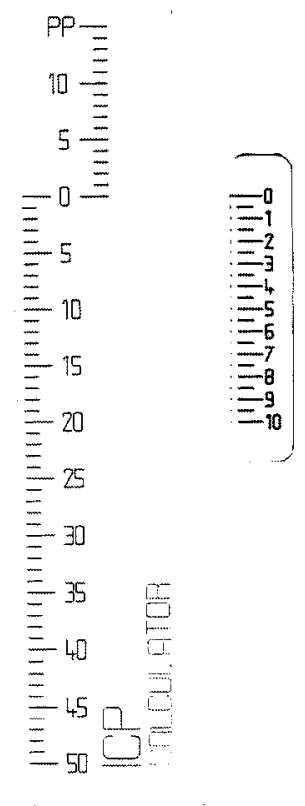


Fig. 5 AFP calculation with the ICP calculator in an AFP recording of a newborn of group I. The large outer bar, containing the small inner bar, is moved along the small bar until the zero of the small inner bar is opposite the equivalent of the measured amplitude. The AFP value is read at the left side of the large outer bar where the bar crosses the horizontal axis of the recording.

With this ICP calculator the AFP calculation from the recordings is standardized.

In each recording all PDC's with a distinct plateau were numbered by the author. The AFP value in each recording was calculated as the average of the AFP values of the first three PDC's. The number of three PDC's was chosen since at least three PDC's with discernable plateaus were always recorded.

In evaluating an AFP measurement an ordinal 5 point scale was used for 'quality' classification. Quality is defined as the readability of the measurement with regard to the distinctness of the plateau. This scale consisted of the following five classes: 1: excellent, 2: good, 3: fair, 4: moderate, 5: bad.

\section{Fontanelle size}

For measuring the fontanelle size, the length (anterior-posterior extent) and the width (lateral extent) were assessed with a measuring tape. The average of the length and the width was defined as 'mean anterior fontanelle size' (mafs, $\mathrm{cm}$ ). This method was introduced by Popich and Smith (1972) and proved to be practical.

\section{Statistical analysis}

For the statistical analysis of the normal range of both the AFP values and the fontanelle sizes, the mean and the standard deviation (SD) were estimated. The normal range is defined as the mean $\pm 2 \mathrm{SD}$. After applying a 'Goodness of fit' test in each of both samples (group I and group II), this test showed that the hypothesis of normality could not be rejected. Therefore the distribution of AFP values was assumed to be approximately Gaussian. Still one should bear in mind that the power of such a test is not very high and that the mean and standard deviation are estimates.

Differences in AFP between the several age classes both in group $I$ and in group II were assessed with the Kruskal-Wallis test, a non-parametric test.

Differences in AFP between group I and group II were assessed with the MannWhitney U-test. 
Correlations between fontanelle size, age, AFP values and quality of AFP measurement were assessed with the Spearmanrank correlation test.

The arithmetic average was used for calculating mean CSFP and mean AFP from the published studies.

A p-value $<0.05$ was considered to be significant.

Data are presented as mean $\pm S D$.

\section{RESULTS}

\section{AFP measurements}

In group I the mean value of AFP was $8.9 \pm 3.0 \mathrm{mmHg}$. Figure 6 shows the AFP values, including the median and the range, for each age class. There was no statistical difference between the various age classes in group $I(p=0.65=N S)$.

In group II the mean value of AFP was $11.0 \pm 1.9 \mathrm{mmHg}$. Figure 7 shows the AFP values, including the median and the range, for each age class. There was no statistical difference between the several age classes in group II $(\mathrm{p}=0.49=\mathrm{NS})$.

Of the total group of 52 infants four infants were measured twice, one infant was measured three times and one infant was measured five times. In Table I the results of these measurements are shown. The number of infants and the number of measurements were both too small to perform any statistical analysis with respect to changes in AFP in relation to time. The trend seems to be that there is no difference in AFP values from 30 days until 210 days of age.

There was a statistically significant difference between the mean AFP value of $8.9 \pm 3.0 \mathrm{mmHg}$ measured in group I and the mean AFP value of $11.0 \pm 1.9 \mathrm{mmHg}$ measured in group II $(p<0.01)$.

\section{mean AFP \\ (mmHg)}

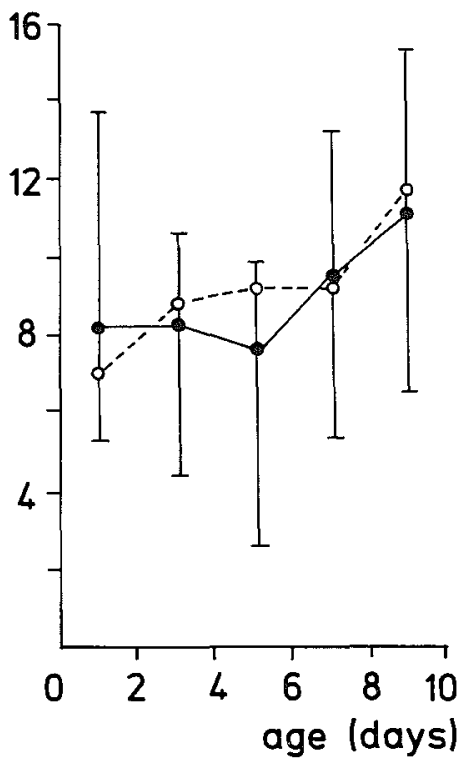

Fig. 6 Mean AFP values ( $\mathrm{mmHg}$ ) for each age class in group I including the median and the range. The AFP values are represented by closed circles, the median is represented by open circles and the range by vertical bars.

\section{Review of the literature concerning normal ICP values in children}

Invasive techniques and non-invasive techniques for measuring CSFP and AFP were studied separately. Reports on invasive ICP measuring techniques for establishing normal values of CSFP in children of various ages are represented in Table II, ranked according to the year of publication.

Although nearly all studies were limited by lack of information concerning the study design, the groups studied, the kind of measuring device and the duration of the measurements, it was tempting to estimate an 'average' level of CSFP from 
mean AFP

(mmHg)

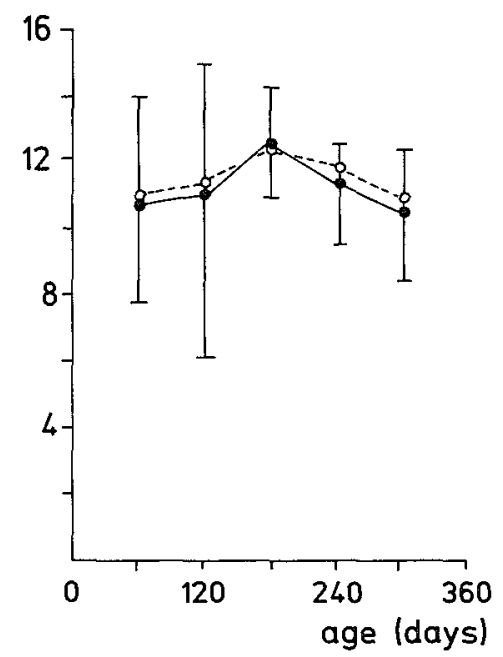

Fig. 7 Mean AFP values $(\mathrm{mmHg})$ for each age class in group II including the median and the range. The AFP values are represented by closed circles, the median is represented by open circles and the range by vertical bars.

Table II. The 'mean' CSFP (arithmetic average) from all studies mentioned was $4.9 \pm 2.7 \mathrm{mmHg}$, which characterizes the distribution of the means per study across all studies.

A curve of normal CSFP values in childhood was published by Paraicz (1982). This is shown in figure 8.

Reports on non-invasive measuring techniques for establishing normal values of AFP in children of various ages are represented in Table III, ranked according to the year of publication.

Although all authors described the techniques used, a number of reports lack information about the studied groups and the duration of the measurements. The 'mean' AFP (arithmetic average) from all studies mentioned was $7.6 \pm 3.2 \mathrm{mmHg}$,

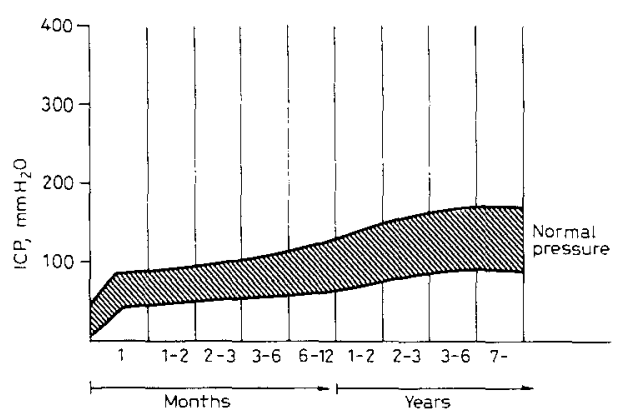

Fig. 8 The normal ICP level in infancy and childhood. (Paraicz 1982). Reproduced with permission from Karger, Basel.

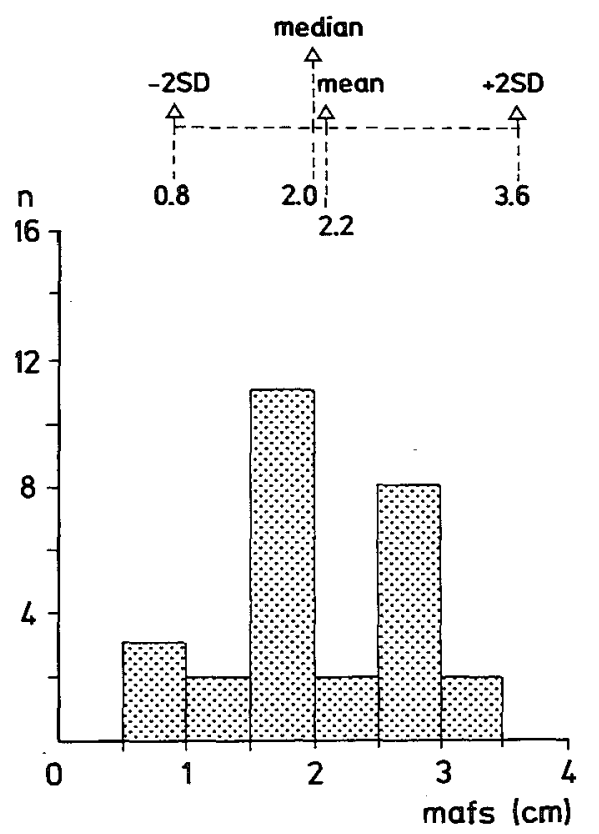

Fig. 9 Mean anterior fontanelle size (mafs), including the mean of the total group, the median and the range $( \pm 2 S D)$ in 28 babies of group $\mathbf{I}$. ( $\mathrm{n}=$ number of newborns).

which characterizes the distribution of the means per study across all studies.

Fontanelle size

In group I the mean fontanelle size was 
TABLE I: AFP values (mmHg) found in six infants of group II during serial AFP measurements, represented according to age class of the infant at the time of the measurement.

\begin{tabular}{|c|c|c|c|c|c|c|}
\hline \multirow{2}{*}{ Patient } & \multicolumn{6}{|c|}{ AFP (mmH) in each age class of 30 days } \\
\cline { 2 - 7 } number & $31-60$ & $61-90$ & $91-120$ & $121-150$ & $151-180$ & $181-210$ \\
\hline 1 & 11 & & 10,7 & & & \\
2 & 12 & 11,6 & & 11,8 & 13 & 12,5 \\
3 & 10,5 & & 13,3 & 11,2 & & \\
9 & 8,1 & 8,4 & & & & \\
30 & 8 & 10,7 & & & & \\
40 & 7,7 & & 10,6 & & & \\
\hline
\end{tabular}

TABLE II: Normal ICP values in children of various ages measured with invasive techniques. $(\mathrm{n}=$ number of individuals in the study, $?=$ number or exact technique not reported).

\begin{tabular}{|c|c|c|c|c|}
\hline $\begin{array}{l}\text { First author, } \\
\text { year of publication }\end{array}$ & $\mathbf{n}$ & $\begin{array}{l}\text { Age (days, or } \\
\text { description) }\end{array}$ & $\begin{array}{l}\text { Pressure } \\
\text { (mmHg) } \\
\pm \mathrm{SD}\end{array}$ & Technique \\
\hline Quincke,1878,1891b & 2 & $80-300$ & $4.0-5.2$ & LP (non-isovolumetric) \\
\hline Sidbury, 1920 & $?$ & newborns & $2-5$ & LP (non-isovolumetric) \\
\hline \multirow[t]{2}{*}{ Levinson, 1928} & ? & newborns & $1.0-6.0$ & LP (non-isovolumetric) \\
\hline & $?$ & infants & $3.0-6.0$ & \\
\hline Munro, 1928 & 55 & $?$ & $2.0-6.0$ & LP (non-isovolumetric) \\
\hline Lups, 1954 & $?$ & children & $2.9-7.4$ & LP (non-isovolumetric) \\
\hline \multirow[t]{2}{*}{ Gerlach, 1967} & ? & infants & $2.9-7.4$ & LP (non-isovolumetric) \\
\hline & & newborns & $0.7-1.0$ & \\
\hline Minns, 1977 & 18 & $<3$ years & $<10$ & VFP (isovolumetric) \\
\hline \multirow[t]{3}{*}{ Paraicz, 1982} & 147 & newborns & 2 & CSFP (technique ?) \\
\hline & & infants & 5 & \\
\hline & & $1-7$ years & $6-13$ & \\
\hline Levene, 1985 & $?$ & term & $3-4$ & $\begin{array}{l}\text { subarachnoid pressure } \\
\text { (isovolumetric) }\end{array}$ \\
\hline Kaiser, 1985, 1986 & 49 & neonates & $2.8 \pm 1.4$ & LP (isovolumetric) \\
\hline Blomquist, 1986 & 15 & 1 month-15 years & $9.8 \pm 3.2$ & LP (isovolumetric) \\
\hline
\end{tabular}

$2.2 \pm 0.7 \mathrm{~cm}$ (range $0.7-3.3 \mathrm{~cm}$ ). In two out of 30 cases the fontanelle was not measured. In figure 9 the mean anterior fontanelle size, including the median and the range ( $\pm 2 S D$ ), is represented. In figure
10 the mafs for each age class is shown. No statistically significant correlation could be established between mean fontanelle size and age class $(p=0.91=N S)$. There was no statistically significant cor- 
TABLE III: Normal AFP values in newborns and in infants measured with non-invasive techniques. ( $\mathrm{n}=$ number of individuals in the study, ? = number, age or pressure not reported).

\begin{tabular}{|c|c|c|c|c|}
\hline $\begin{array}{l}\text { First author, } \\
\text { year of publication }\end{array}$ & $\mathrm{n}$ & $\begin{array}{l}\text { Age (days, or } \\
\text { description) }\end{array}$ & $\begin{array}{l}\mathrm{AFP} \\
(\mathrm{mmHg}) \\
\pm \mathrm{SD}\end{array}$ & Technique \\
\hline Davidoff, 1959 & $?$ & $?$ & $13.6-20.4$ & $\begin{array}{l}\text { Adapted Schiotz } \\
\text { tonometer }\end{array}$ \\
\hline Barashnev, 1966 & ? & prematures & $5.2 \pm 6.6$ & Purins Tambour \\
\hline Robinson, 1977 & 91 & $1-2$ & $7.9 \pm 2.3$ & Transducer \\
\hline Salmon, 1977 & 35 & $1-3$ & $7.4 \pm 1.5$ & Transducer \\
\hline Vidyasagar, 1977 & 30 & $1-7$ & $7.5 \pm 0.2$ & $\begin{array}{l}\text { Ladd (fiber-optic } \\
\text { device) }\end{array}$ \\
\hline Hayashi, 1978 & 49 & $0-\geqslant 30$ & $5.3-10.8$ & Marey Tambour \\
\hline Donn, 1978 & 18 & $\begin{array}{l}\text { prematures } \\
\text { mean } 32 \text { weeks }\end{array}$ & $9-17.9$ & Ladd \\
\hline Holbrook, 1978 & 4 & infants & 7.4-19.1 & Ladd \\
\hline Vidyasagar, 1978 & 39 & term & $7.5 \pm 0.3$ & Ladd \\
\hline Raju, 1980 & 7 & preterm & $7.0 \pm 0.6$ & Ladd \\
\hline Huch, 1979 & 130 & term & $7.9 \pm 2.8$ & Transducer \\
\hline Gaab, 1980 & 6 & $3-210$ & $5 \pm 2$ & Ladd \\
\hline Welch, 1980 & 28 & $0-210$ & $3.3 \pm 0.8$ & $\begin{array}{l}\text { Indirect observation } \\
\text { of fontanelle }\end{array}$ \\
\hline Von Wild, 1980, 1981 & 27 & $\begin{array}{l}\text { newborns and } \\
\text { infants }\end{array}$ & $0-5$ & Ladd \\
\hline Daniels, 1981 & 78 & $0-90$ & $7.5 \pm 1.8$ & Tonometer \\
\hline Peabody, 1981 & $\begin{array}{r}103 \\
5\end{array}$ & $\begin{array}{l}\text { term } \\
\text { preterm }\end{array}$ & $\begin{array}{l}7.9 \pm 3.0 \\
6.7 \pm 4.0\end{array}$ & Transducer \\
\hline Philip, 1981 & $\begin{array}{r}120 \\
53\end{array}$ & $\begin{array}{l}0-3 \\
\text { preterm }\end{array}$ & $\begin{array}{l}8.8 \pm 1.9 \\
5.4-12.5\end{array}$ & Fiber-optic device \\
\hline Menke, 1982 & 52 & $0-3$ & $7.0 \pm 1.9$ & Pneumo-tonometer \\
\hline Bada, 1983 & $\begin{array}{l}30 \\
33\end{array}$ & $\begin{array}{l}\text { term newborns } \\
\text { preterm }\end{array}$ & $\begin{array}{l}7.6-8.0 \\
\pm 2.0 \\
6.5\end{array}$ & Transducer \\
\hline Easa, 1983 & 35 & $\begin{array}{l}1-3 \\
\text { preterm }\end{array}$ & $\begin{array}{l}5.3 \pm 1.3 \\
5.1 \pm 0.9\end{array}$ & Pneumo-tonometer \\
\hline Perlman, 1983 & 35 & $\begin{array}{l}\text { prematures } \\
26-35 \text { weeks }\end{array}$ & $4.3 \pm 1.0$ & Ladd \\
\hline Ivan, 1983 & $\begin{array}{r}101 \\
35 \\
36\end{array}$ & $\begin{array}{l}0-\leqslant 4 \\
4-28 \\
34-219\end{array}$ & $\begin{array}{l}8.7 \pm 2.7 \\
9.8 \pm 0.9 \\
11.2 \pm 0.8\end{array}$ & Ladd \\
\hline Walsch, 1983 & 17 & infants & 3.5 & Ladd \\
\hline Brömme, 1985 & $\begin{array}{l}30 \\
50\end{array}$ & $\begin{array}{l}1-6 \\
310-360\end{array}$ & $\begin{array}{l}12-15 \\
11\end{array}$ & Transducer \\
\hline
\end{tabular}




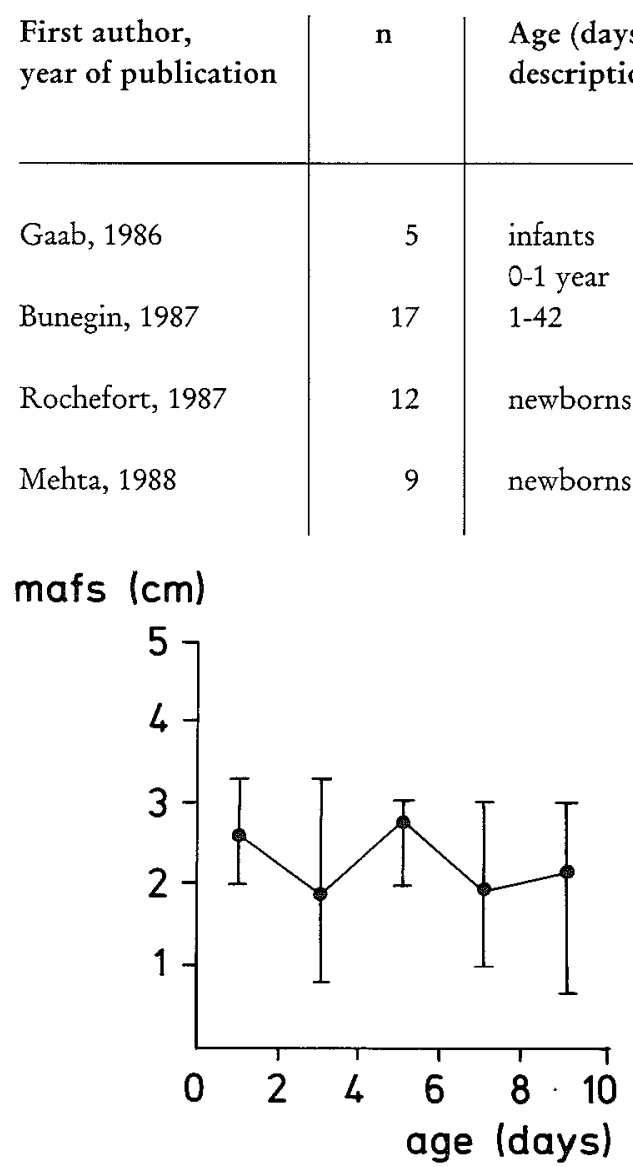

Fig. 10 Mean anterior fontanelle size (mafs), including the range, in each age class of group I. The mafs is represented by closed circles, the bars represent the range.

relation between fontanelle size and AFP level $(p=0.98=N S)$.

In group II the mean fontanelle size was $2.4 \pm 0.9 \mathrm{~cm}$ (range $1.3-6.0 \mathrm{~cm}$ ). In figure 11 the mean anterior fontanelle size, including the median and the range $( \pm 2$ $\mathrm{SD}$ ), is presented. In figure 12 the mafs for each class is shown.

There was a statistically significant correlation between mean fontanelle size and age class $(p<0.01)$. The correlation between fontanelle size and AFP level was

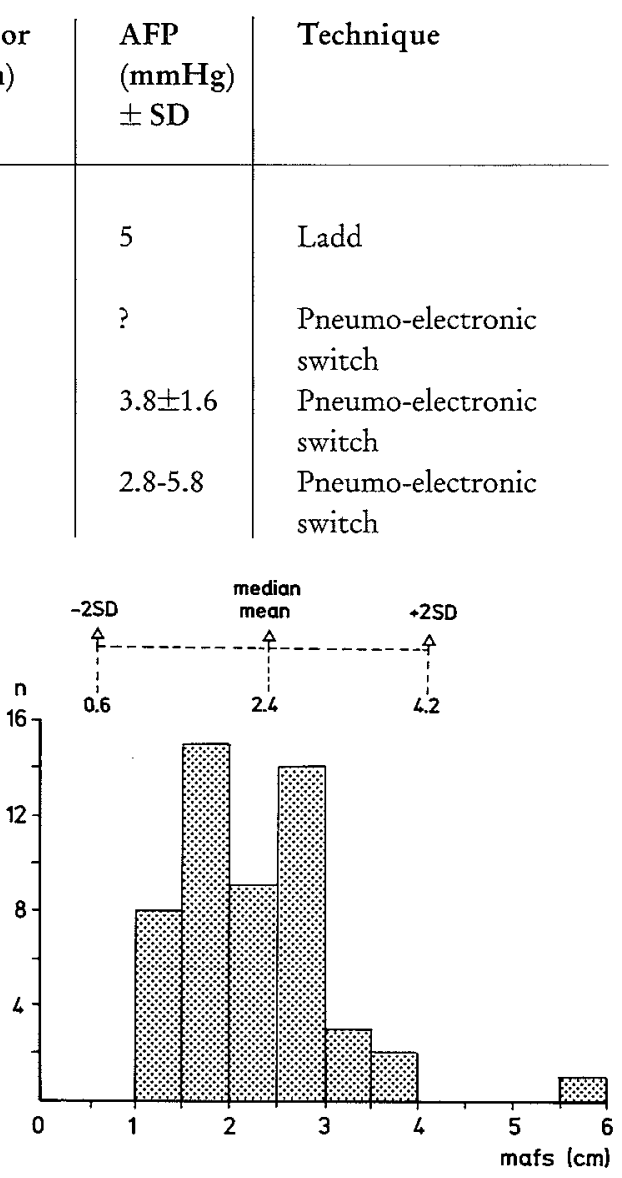

Fig. 11 Mean anterior fontanelle size (mafs), including the mean of the total group, the median and the range $( \pm 2 S D)$ in 52 infants of group II. ( $n=$ number of infants).

not statistically significant $(\mathrm{p}=0.67=$ NS).

In group I there was a statistically significant correlation between the fontanelle size and the quality of the AFP recording $(p=0.03)$. In group II the correlation between the fontanelle size and the quality of the AFP recording was not statistically significant $(p=0.1=N S)$. 


\section{DISCUSSION}

AFP values in normal newborns and infants

From the end of the nineteenth century until now an extensive number of reports have appeared dealing with normal values of ICP in children. It was necessary to distinguish between invasive methods and non-invasive methods. This distinction was based on ICP monitoring techniques performed in adults in which CSFP tends to read slightly lower than EDP (Coroneos et al. 1972, 1973, Jörgensen and Riishede 1972, Sundbärg and Nornes 1972, Zierski 1980). This is probably due to mechanical forces in the dura, although the investigators all claim they had eliminated these forces. Therefore, the application of any epidural transducer should be done according to the co-planarity principle (Schettini and Walsh 1974). Anterior fontanelle pressure monitoring can be compared to epidural pressure monitoring since only the skin, including subcutaneous tissue, is interposed between the transducer and the dura. As is shown in Tables II and III the normal values of CSFP in children are lower than the normal values of AFP, as is also found in ICP monitoring in adults (CSFP versus EDP).

The fixation of the measuring device to the anterior fontanelle is very important. If collodion or plaster of Paris is used, the skin could be slightly pulled off the underlying tissues and the dura (Walsch and Logan 1983, McIntosh 1983). This might cause a lower AFP value. Self adhesive tape (Von Wild and Pörksen 1980, Gaab et al. 1980, 1981) and foam pads (Hill and Volpe 1981a) could either pull off the skin or compress the transducer to the skin giving lower or higher AFP values respectively. Special holders (handheld or fixed with adhesives) were tested, especially regarding the Ladd transducer for performing con-

\section{mafs $(\mathrm{cm})$}

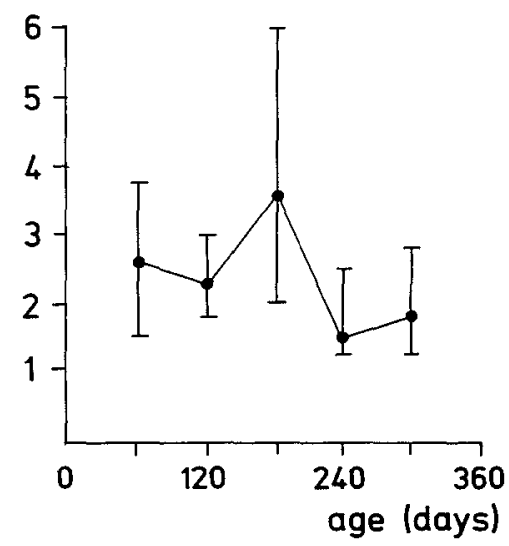

Fig. 12 Mean anterior fontanelle size (mafs), including the range in each age class of group II. The mafs is represented by closed circles, the bars represent the range.

tinuous measurements (Walsh and Logan 1983, Myerberg et al. 1980). An ECG electrode adhesive disk appeared useful (Donn and Philip 1978), but in continuous monitoring procedures the fixation had to be regularly checked. All authors recommend shaving the fontanelle. Abundant hair causes a shift of the transducer in spite of proper fixation. Chapter 3 describes how the RTT in the special fontanelle adaptor is maintained in position at a fixed depth by means of the special fixation frame. With this method application forces were excluded as much as possible. Horbar et al. (1980), Gaab et al. (1980), Brömme et al. (1985) and Kaiser and Whitelaw (1987a) paid thorough attention to these application forces. They applied the Ladd device or strain gauge transducers to the fontanelle and found that the amount of force necessary in applying a transducer to the fontanelle influenced the measured AFP value. Nearly all authors, except Brömme et al. (1985), applied the transducers directly to the fontanelle without special adaptors or they used an adap- 
tor which rested on the fontanelle. Brömme used an adaptor which rested on the bony surroundings of the fontanelle. However, the fixation method consisted of rubber bands wrapped around the head in the occipito frontal region, and so application forces still interfered with the pressure measurement.

In the present study the adaptor of the RTT rested on the bony surroundings of the fontanelle and according to the balance of forces any surplus of application force runs off to the skull and AFP is reliably measured. The normal values of AFP in this study, both in group I and group II, though slightly higher than in most reports, are within the range of normal values obtained with non-invasive techniques (Vidyasagar et al. 1978, Philip et al. 1981, Menke et al. 1982, Ivan and Badejo 1983, Brömme et al. 1985).

As mentioned before, non-isovolumetric methods for measuring ICP were mentioned separately from isovolumetric methods. This was done because using non-isovolumetric manometers influences pressure since the CSF volume required to fill the apparatus will lower the pressure. Minns (1977) and Kaiser and Whitelaw (1986) measured ventricular fluid pressure and lumbar pressure isovolumetrically by connecting the CSF filled tubing directly to a Gaeltec pressure transducer. The CSFP values measured with this isovolumetric technique however did not differ much from the earlier reports in which the authors used non-isovolumetric methods. This may indicate that normal newborns and infants are in the flat part of their volume-pressure curve (see chapter 5 , figure 14) and therefore loss of CSF volume has only a minor influence on the measured pressure (Löfgren et al. 1973, Löfgren 1975).
In comparing the various techniques for AFP monitoring, the technical specifications of the various devices should not be forgotten. Every device has its own systematic error, its own zero-drift and its own calibration problems. The dynamic response of the devices also varies. The RTT has advantages because of the fast dynamic response, the small zero-drift, the fact that no calibration problems exist and because of its ease of application.

\section{Relation AFP-age}

In this study a significant difference in the AFP level between newborns (group I) and infants (group II) was established. This is in accordance with reports from others using invasive techniques for measuring ICP in children of various ages. In preterm and term babies lower pressures were recorded than in older children (Vidyasagar and Raju 1977, Welch 1978, Philip et al. 1981). Paraicz (1982) recorded CSF pressures in 147 infants and children and constructed from these values a curve, which is shown in figure 10. With noninvasive techniques also an age/pressure relationship was established (Robinson et al. 1977, Peabody et al. 1981, Easa et al. 1983). Methods for measuring AFP vary, as do the fixation procedures. This could explain some of the differences in recorded AFP levels. However, if with the same device a significant difference in AFP level is assessed then this difference might be real (Philip et al. 1981).

It is not exactly clear why infants and children have lower ICP levels than adults. The transition of low level into 'adult' level is supposed to occur around 6 years of age (Merritt and Fremont Smith 1937). Paraicz (1982) suggested that the 'variable plasticity' of the infant's skull was the reason for the pressure differences. However this is considered a rather vague 
explanation.

Under normal circumstances an equilibrium exists within the craniospinal compartment called the steady state condition, implying equal CSF-formation and CSF absorption rate (Marmarou et al. 1975, 1978). The steady state condition is represented as:

$$
\mathrm{Pss}_{\mathrm{s}}=\mathrm{RoF}_{\mathrm{i}}+\mathrm{Pd}
$$

$$
\begin{aligned}
\text { where Pss }= & \text { steady state pressure } \\
& (\mathrm{mmHg}) \\
\mathrm{Ro}= & \text { outflow resistance, being } \\
& \text { resistance to CSF } \\
& \text { absorption }(\mathrm{mmHg} / \mathrm{ml} / \mathrm{min}) \\
\mathrm{Fi}= & \text { CSF formation rate } \\
& (\mathrm{ml} / \mathrm{min}) \\
\mathrm{Pd}= & \text { dural sinus pressure } \\
& (\mathrm{mmHg})
\end{aligned}
$$

The explanation for the lower pressure values in young children might be found in some of the parameters of the steady state condition.

Ro is considered constant under normal conditions (Shapiro et al. 1980, demonstrated a slightly larger Ro in children than in adults).

$\mathrm{Fi}_{1}$ is also considered constant under normal conditions. In children this is assessed to be 0.30 to $0.35 \mathrm{ml} / \mathrm{min}$ (Cutler et al. 1968, Lorenzo et al. 1970). In adults the CSF formation rate is reported to be 0.40 $\mathrm{ml} / \mathrm{min}$ (Rubin et al. 1966).

$\mathrm{Pd}$ is not easily measured, often it is approximated by the central venous pressure or assumed to be equal to zero, both in adults and in children.

The several parameters of the steady state condition were not evaluated at the same time in the reported studies. However, considering an equal difference in pressure (Pss-Pd), the results of the studies on both $\mathrm{Ro}$ and $\mathrm{Fi}$ are in accordance with one another. Probably the difference in $\mathrm{Fi}$ value between adults and children in the steady state condition formula could be an explanation for the lower pressure values in young children.

Regarding the pressure values found in the group of newborns and in the group of infants we could not establish any significant relationship between pressure level and age class within each group. Others, however, reported differences in pressure occurring particularly soon after birth. Lopez-Ibor et al. (1982) and Donn and Philip (1978) found a downward trend in ICP during the first three days after birth in both preterm and term born babies. Williams et al. (1977) observed a close correlation between the so-called physiological weight loss and the reduction of the head circumference in the postnatal period and ascribed this to the concomitant loss of CSF and cerebral interstitial fluid. This loss of CSF could possibly explain the downward trend in ICP during the first days of life.

De Souza et al. (1976) observed shrinking of the head after birth. Welch (1980) performed serial indirect pressure measurements in babies with birth weights above and below 2500 gram and observed a decrease in ICP followed by an increase in ICP, which was ascribed to brain-turgor, decreasing after birth and recovering during the first three weeks after birth. In this study the number of infants in each class was too small to draw any conclusions on ICP trend during the first days of life. Since AFP monitoring with the RTT is easily performed a prospective study on ICP trend after birth could be one of the aims of further research.

\section{Normal values of fontanelle size}

The mean values of fontanelle size both in group I and group II were equal to the 
values found by Popich and Smith (1972). In group I no correlation between fontanelle size and age could be established. This group however consisted of newborns up to 9 days of age and it was not expected that the fontanelle size should change during such a short time.

In group II a significant correlation between fontanelle size and age class could be assessed. This is in accordance with the closure of the fontanelle during the first year of life (Aisenson 1950).

We considered the method of Popich and Smith (1972) practical in clinical use.

Fontanelle size with reference to anterior fontanelle pressure and measurement quality Both in group I and in group II no correlation between the fontanelle size and the pressure level could be established. In group I there'was a significant correlation between the fontanelle size and the quality of the AFP recording, giving rise to a poorer readability of the recording when the fontanelle size was smaller. This was expected since in larger fontanelles it was usually easier to perform PDC's. Further- more the fontanelle size in group I was just above the size necessary to perform PDC's with the RTT (mafs $>0.5 \mathrm{~cm}$ ). In group II no significant correlation could be found between the fontanelle size and the quality of the AFP recordings. The fontanelle sizes in this group were slightly larger than in group I (mafs above $1 \mathrm{~cm}$ ). A factor which could not be taken into account however, was the stillness of the child during each recording. We know that in a still and quiet baby a better measurement quality is obtained. Since this is a source of error the correlation between the fontanelle size and the measurement quality might be distorted. Therefore no definite conclusions could be drawn from these observations. In contrast to the significant correlation between the fontanelle size and the quality of the AFP recording in group I, no significant correlation could be established between these two variables in group II. This means that as long as the fontanelle size is of $1 \mathrm{~cm}$ diameter or more an AFP recording can be performed, and the factor most likely to affect the recording is the degree of stillness and quietness of the baby.

\section{CONCLUSIONS}

1 Normal AFP values recorded with the RTT were within the range of AFP values reported in the literature and obtained with other non-invasive methods.

2 The AFP monitoring technique described in this thesis can be considered technically feasible and reliable for measuring AFP in newborns and infants.

3 The difference in mean AFP values between newborns (group I) and infants (group II) was statistically significant and was in accordance with age dependent AFP levels reported by others.

4 The mechanism of the lower ICP levels in young children compared to the higher adult ICP values is still not well understood.

5 Normal values of AFP, as reported in the literature, are slightly higher than normal values of CSFP in children. This is comparable to ICP monitoring in adults where EDP is slightly higher than CSFP. 6 The fontanelle size in babies could be easily assessed with Popich's method. The fontanelle sizes in newborns (group I) and in infants (group II) were in the same range as those reported by Popich and Smith (1972).

7 The fontanelle size in newborns and in infants did not correlate to the AFP level. 


\section{ANTERIOR FONTANELLE PRESSURE MEASUREMENTS IN PATIENTS \\ INTRODUCTION}

After thoroughly testing the RTT in vitro, with special attention to sealing and zero drift, the transducer was ready, not only for implantation in patients with closed sutures, but also for anterior fontanelle pressure monitoring (De Jong et al. 1975, 1979, 1983, 1984, De Lange et al. 1978, Maas and De Jong 1986a, Plandsoen et al. 1986a,b, 1987, 1988, 1989). In chapter 3 was described how to perform AFP monitoring with the RTT in the special skull adaptor.

After establishing normal values of AFP and comparing these to the values found in the literature (described in chapter 4), we considered which technique was most reliable in clinical practice. AFP measurements were performed in newborns and infants admitted to the neurological, neurosurgical and pediatric ward of the
University Hospital of Rotterdam. We wondered whether the RTT could monitor high AFP levels in patients with clinical signs of raised ICP. Futhermore we wondered whether AFP monitoring was useful in pediatric patients with possible signs of raised ICP, such as bulging anterior fontanelle or vomiting.

Subsequently we investigated possibilities of performing continuous AFP measurements, not only to test the fixation of the device but also to find out whether abnormal pressure fluctuations could be recorded.

The relationship between this non-invasive ICP monitoring technique and invasive techniques for measuring CSFP by the lumbar or the ventricular method was studied by performing a number of comparative measurements.

\section{MATERIAL AND METHODS}

In 141 patients 253 AFP measurements were performed. Fifty-five of these patients were part of a pilot study which was reported earlier (Plandsoen et al. 1986b). In each patient at least one short term AFP measurement was performed which consisted of at least 3 PDC's with discernable plateaus.

The recordings in all 141 patients were performed from November 1982 to March 1988. The patients were categorized into diagnostic classes according to a central nervous system localization (CNS) or a non central nervous system localization (NCNS). In Table I the diagnostic classes, the number of patients in each class and the number of AFP measurements are shown. Classification was based on a combination of medical history, clinical signs and ancillary examinations (laboratory, CT scan and ultrasound of the skull).

Of the total number of 141 patients 53 were females and 88 were males.

In the CNS diagnostic classes 23 premature babies are included (27-36 weeks ge- 
TABLE I: Number of patients and AFP measurements categorized according to diagnostic classes.

\begin{tabular}{|c|c|c|c|c|c|c|c|c|}
\hline \multirow[b]{2}{*}{ Diagnostic class } & \multicolumn{4}{|c|}{ CNS } & \multicolumn{4}{|c|}{ NCNS } \\
\hline & $\mathrm{n}_{\mathrm{par}}$ & $\mathrm{n}_{\mathrm{AFP}}$ & $\mathrm{n}_{\text {patc }}$ & $\mathrm{n}_{\mathrm{AFP}} \mathrm{c}$ & $\mathrm{n}_{\mathrm{par}}$ & $\mathrm{n}_{\mathrm{AFP}}$ & $\mathrm{n}_{\text {patc }}$ & $\mathrm{n}_{\mathrm{AFPc}}$ \\
\hline Inflammation & 6 & 9 & 3 & 4 & 1 & 1 & - & - \\
\hline $\begin{array}{l}\text { (Cardio)vascular } \\
\text { disorders + haemorrhages }\end{array}$ & 25 & 65 & 14 & 22 & 10 & 12 & 2 & 2 \\
\hline $\begin{array}{l}\text { Congenital and } \\
\text { hereditary disorders }\end{array}$ & 78 & 142 & 26 & 45 & 10 & 13 & 1 & 4 \\
\hline Miscellaneous & 8 & 8 & 2 & 2 & 3 & 3 & - & - \\
\hline Total & 117 & 224 & 45 & 73 & 24 & 29 & 3 & 6 \\
\hline
\end{tabular}

CNS located within the central nervous system

NCNS not located within the central nervous system

$n_{\text {pat }} \quad$ number of patients

$n_{A F P} \quad$ number of AFP measurements

$n_{\text {patc }} \quad$ number of patients with longterm ( $=$ continuous) AFP measurements (continuous is defined as at least 30 minutes)

$n_{A F P_{c}} \quad$ number of continuous AFP measurements

stational age). Thirteen babies were in the (cardio)vascular disorders and haemorrhages diagnostic class (all 13 with the diagnosis of perinatal haemorrhages), nine babies in the congenital and hereditary disorders diagnostic class (one with the diagnosis of macrocephaly and internal hydrocephalus on CT-scan, three with macrocephaly and external hydrocephalus on CT-scan, one with macrocephaly and subdural effusions on CT-scan, one with arachnoid cysts, two with aquaductal stenosis, one with triventricular hydrocephalus of unknown cause) and one baby in the miscellaneous diagnostic class, diagnosed as non-specific psychomotor retardation.

In the NCNS diagnostic classes were four premature babies (27-36 weeks gestational age). Of these four babies one was in the cardiovascular disorders and haemorrhages diagnostic class (with the diagnosis of atrio-ventricular block), two in the congenital and hereditary disorder diagnostic class (one with the diagnosis of cystic fibrosis, one with the diagnosis of diaphragmatic hernia), and one in the miscellaneous diagnostic class (merely diagnosed as premature).

In 48 patients 79 continuous AFP measurements were performed which lasted for 30 minutes or more. In some patients the respiration was also recorded to evaluate pathological pressure waves. The numbers of these measurements are shown in Table I. 
The average AFP value of a continuous recording was established from the mean of the recorded pulsations. Only at the start of a continuous AFP measurement were the standard three PDC's obtained. The mean AFP was estimated on visual inspection of the recording and by calculation (see below).

It is important to evaluate a continuous ICP recording not only for the level of pressure but also for the occurrence of Awaves and $\mathrm{B}$-waves. $\mathrm{A}$-waves are defined as large plateau like formations occurring at intervals of varying length usually with an amplitude range from $50-100 \mathrm{mmHg}$ and a duration of 5-20 minutes (Lundberg 1960). B-waves are defined as rhythmic oscillations occurring more or less regularly at a frequency varying from $1 / 2-2$ per minute and an amplitude from discernable to $50 \mathrm{mmHg}$.

The analysis of the continuous recordings consisted of the following steps:

- defining the total duration of the recording,

- discarding parts of the recording disturbed by movement artifacts,

- estimating the average level of AFP on visual inspection,

- calculating the level of AFP from the average of 10 measuring points taken every 30 minutes from a part of the recording without movement artifacts, using the ICP calculator (if the recording lasted less than 5 hours the 10 measuring points were taken with shorter time intervals),

- identifying B-waves and A-waves and defining the amplitude and the duration of those waves,

- calculating the percentage of B-waves by multiplying the ratio of the total duration of B-waves in the interpretable parts of the recording and the total interpretable parts of the recording by 100 , - determining the amplitude of the Bwaves by calculating the average of 5 well recorded B-waves in 5 different periods of the recording.

- calculating the amplitude of the Awaves by defining for each wave separately the pressure at the base level and the pressure at the peak level of the wave.

The Wilcoxon matched pairs signed rank test was performed to detect a difference in the level of AFP judged by visual inspection and by calculation with the ICP calculator.

In 23 patients a total number of $52 \mathrm{AFP}$ measurements was performed before and after 26 shunting procedures to evaluate the effect of an operation on the level of ICP. The patients selected had AFP measurements performed within one month before and within one month after the operation.

One patient was measured twice and one patient was measured three times, both because of a shunt dysfunction. The Wilcoxon matched pairs signed rank test was used to test whether the difference between the pre and post operative AFP values was statistically significant.

In 13 patients of the CNS diagnostic classes 16 comparative measurements were done between AFP and VFP or LP. The techniques were puncture of the fontanelle, the ventricle (during operation), the Rickham reservoir, or the lumbar space with a needle connected to either an open end or closed manometer. In Table II the number of patients and comparative measurements in each diagnostic class is represented. If necessary the children were sedated with chloral hydrate. This drug does not influence ICP (Scanarini et al. 1982).

\section{RESULTS}

The results of the initial short term AFP measurements in the total group of 141 
TABLE II: Number of patients and comparative measurements in the CNS diagnostic classes. In the 5 patients with perinatal haemorrhages 8 measurements were performed.

\begin{tabular}{|c|c|}
\hline Diagnostic class & Diagnosis \\
\hline Inflammation & - bacterial meningitis \\
\hline (Cardio)vascular & - choroid plexus papilloma \\
\hline disorders and haemorrhages & $\begin{array}{l}\text { - perinatal haemorrhages (intracerebral, } \\
\text { intraventricular and subdural bleeding) }\end{array}$ \\
\hline $\begin{array}{l}\text { Congenital and } \\
\text { hereditary disorders }\end{array}$ & $\begin{array}{l}\text { - myelodysplasia } \\
\text { - aquaductal stenosis } \\
\text { - macrocephaly and internal } \\
\text { hydrocephalus on CT-scan } \\
\text { - macrocephaly and external } \\
\text { hydrocephalus on CT-scan } \\
\text { - familial macrocephaly without } \\
\text { CT-scan abnormality }\end{array}$ \\
\hline
\end{tabular}

patients are shown in Tables III and IV, divided according to a CNS (Table III) or a NCNS localization (Table IV). The patients were ordered according to diagnostic classes and diagnosis.

The age of the patients in the CNS diagnostic classes at the time of the first AFP measurement ranged from 30 weeks gestational age to 24 months calendar age. One exception consisted of an 8 year old boy with multiple skull defects caused by multiple operations for correction of a craniosynostosis. This boy had also been treated for hydrocephalus with a shunt.

With the RTT in the fontanelle adaptor, pressure was measured on a skull defect. This is comparable with measuring on a false fontanelle (Forrest and Tsingoglu 1968, Harbaugh and Saunders 1984). In this patient reproducible PDC's could be recorded and a successful continuous pressure recording of 10 hours and 30 minutes was performed of which 8 hours and 15 minutes could be interpreted. Fi- gures 1 and 2 show a PDC measured in the short term AFP recording and pressure fluctuations in part of the continuous AFP recording.

With the RTT high pressures could also be recorded as is shown in figure 3 showing the PDC of a short term AFP measurement performed in a comatose patient with bilateral subdural haemorrhages (patient 99).

The age of the patients in the NCNS diagnostic classes at the time of the first AFP measurement ranged from 30 weeks gestational age to $11 \frac{1}{2}$ months calendar age.

\section{Continuous measurements}

The maximal duration of the continuous AFP measurements in the patients of the CNS diagnostic classes was 39 hours and 15 minutes. The maximal duration of the continuous measurements in the NCNS diagnostic classes was 10 hours and 35 minutes. 
TABLE III: CNS diagnostic classes, diagnosis, number of patients and AFP values $\pm \mathrm{SD}(\mathrm{mmHg})$.

\begin{tabular}{|c|c|c|c|}
\hline Diagnostic class & Diagnosis & $\mathbf{n}$ & AFP \\
\hline Inflammation & - bacterial meningitis & 6 & $19.9 \pm 7.7$ \\
\hline (Cardio)vascular & - arterio venous malformation & 1 & 17.5 \\
\hline disorders & - choroid plexus papilloma & 1 & 23.6 \\
\hline \multirow[t]{3}{*}{ and haemorrhages } & - Sturge Weber syndrome & 1 & 23.3 \\
\hline & $\begin{array}{l}\text { - perinatal haemorrhages (intraventricular, } \\
\text { subdural, subarachnoid, subependymal, } \\
\text { and parenchymal bleeding) }\end{array}$ & 21 & $17.5 \pm 9.3$ \\
\hline & - haemorrhagic shock encephalopathy & 1 & 7.9 \\
\hline \multirow{17}{*}{$\begin{array}{l}\text { Congenital and } \\
\text { hereditary } \\
\text { disorders }\end{array}$} & - craniosynostosis & 11 & $18.0 \pm 5.7$ \\
\hline & - myelodysplasia & 20 & $16.1 \pm 7.0$ \\
\hline & - agenesis of corpus callosum & 2 & $12.7 \pm 0.9$ \\
\hline & - porencephalic cysts & 2 & $19.1 \pm 6.2$ \\
\hline & - arachnoid cysts & 2 & $14.6 \pm 6.4$ \\
\hline & - aquaductal stenosis & 2 & $14.2 \pm 8.8$ \\
\hline & - Dandy Walker malformation & 1 & 11.4 \\
\hline & - non-specific leucodystrophy & 1 & 5.3 \\
\hline & - oculomotor apraxia & 1 & 12.7 \\
\hline & - chromosomal disorder and hydrocephalus & 1 & 24.8 \\
\hline & - Gorlin's disease with hydrocephalus & 1 & 21.4 \\
\hline & $\begin{array}{l}\text { - triventricular hydrocephalus of } \\
\text { unknown cause }\end{array}$ & 4 & $15.6 \pm 7.3$ \\
\hline & $\begin{array}{l}\text { - quadriventricular hydrocephalus of } \\
\text { unknown cause }\end{array}$ & 7 & $25.0 \pm 9.3$ \\
\hline & $\begin{array}{l}\text { - macrocephaly with internal } \\
\text { hydrocephalus on CT scan }\end{array}$ & 6 & $16.9 \pm 3.4$ \\
\hline & $\begin{array}{l}\text { - macrocephaly with external hydrocephalus } \\
\text { on CT scan }\end{array}$ & 8 & $17.1 \pm 3.6$ \\
\hline & $\begin{array}{l}\text { - macrocephaly with subdural } \\
\text { effusions on CT scan }\end{array}$ & 2 & $10.6 \pm 1.2$ \\
\hline & $\begin{array}{l}\text { - macrocephaly without CT-scan abnormality } \\
\text { (including familial macrocephaly) }\end{array}$ & 7 & $13.5 \pm 2.6$ \\
\hline \multirow[t]{4}{*}{ Miscellaneous } & - seizures & 2 & $19.0 \pm 0.9$ \\
\hline & - headtrauma and skullfractures & 1 & 27.3 \\
\hline & - neuroblastoma & 1 & 12.8 \\
\hline & - non-specific psychomotor retardation & 4 & $13.8 \pm 2.3$ \\
\hline
\end{tabular}


TABLE IV: NCNS diagnostic classes, diagnosis, number of patients and AFP values $\pm \mathrm{SD}(\mathrm{mmHg})$.

$\begin{array}{llll}\text { Diagnostic class } & \text { Diagnosis } & \mathbf{n} & \text { AFP } \\ \text { Inflammation } & \text { - gastro-enteritis } & & 12.0 \\ \text { (Cardio)vascular } & \text { - atrio-ventricular block in } & 1 & 14.1 \\ \text { disorders and } & \text { Jervell Lange Nielsen disease } & 1 & \\ \text { haemorrhages } & \text { - coarctation of the aorta } & & 14.6 \\ & \text { - transposition of great arteries } & 1 & 19.3 \\ & \text { - Tetralogy of Fallot } & 5 & 19.5 \pm 4.4 \\ & \text { - Klippel Trenaunay syndrome } & 1 & 14.1 \\ \text { Congenital and } & \text { - haemangioma of the knee } & 1 & 14.2 \\ \text { hereditary } & \text { - cleft lip and palate } & 1 & 14.1 \\ \text { disorders } & \text { - diaphragmatic hernia } & 2 & 12.5 \pm 0.9 \\ & \text { - omphalocele } & 1 & 16.3 \\ & \text { - oesophageal atresia } & 1 & 18.1 \\ & \text { - cystic fibrosis } & 1 & 17.3 \\ & \text { - hermaphroditism } & 1 & 11.7 \\ & \text { - osteogenesis imperfecta } & 1 & 32.0 \\ & \text { - sickle cell disease } & 1 & 17.8 \\ & \text { - hydronefrosis } & 1 & 14.9 \\ & \text { - near sudden infant death syndrome } & 1 & 12.0 \\ & \text { - premature birth } & 1 & 3.2 \\ \text { Miscellaneous } & \text { - hypertrophic pyloric stenosis } & 1 & 16.9 \\ & & 24 & \end{array}$

The Wilcoxon test showed no significant difference between the visually estimated level of AFP and the calculated level of AFP in the 79 continuous AFP recordings $(\mathrm{p}=0.2=\mathrm{NS})$.

With this AFP recording technique it was possible to monitor $\mathrm{B}$-waves and $\mathrm{A}$-waves. In the CNS diagnostic classes B-waves were detected in 57 recordings in 38 patients. The percentages of registered Bwaves varied from 5.3 to 96.4 . The greatest percentage of B-waves (96.4) was found in the child with bilateral subdural haemorrhages caused by multiple minor head injuries (patient 99, described in chapter 7). Figure 4 shows a part of the continuous
AFP recording in this patient. The amplitude of the B-waves in the CNS diagnostic classes ranged from 1.7 to 11.4 $\mathrm{mmHg}$. The highest amplitude was found in the above mentioned patient with bilateral subdural haemorrhages (patient 99). $B$-waves with an amplitude of more than $3.5 \mathrm{mmHg}$ were registered in patients with an AFP level of more than $15 \mathrm{mmHg}$. In these patients also the largest percentage (more than $20 \%$ of the recording time) was found. In a comatose patient (patient 73: haemorrhagic shock encephalopathy) a B-wave occurring percentage of 95 was recorded with a $\mathrm{B}$-wave amplitude of 5.4 $\mathrm{mmHg}$ while AFP was below $15 \mathrm{mmHg}$. 


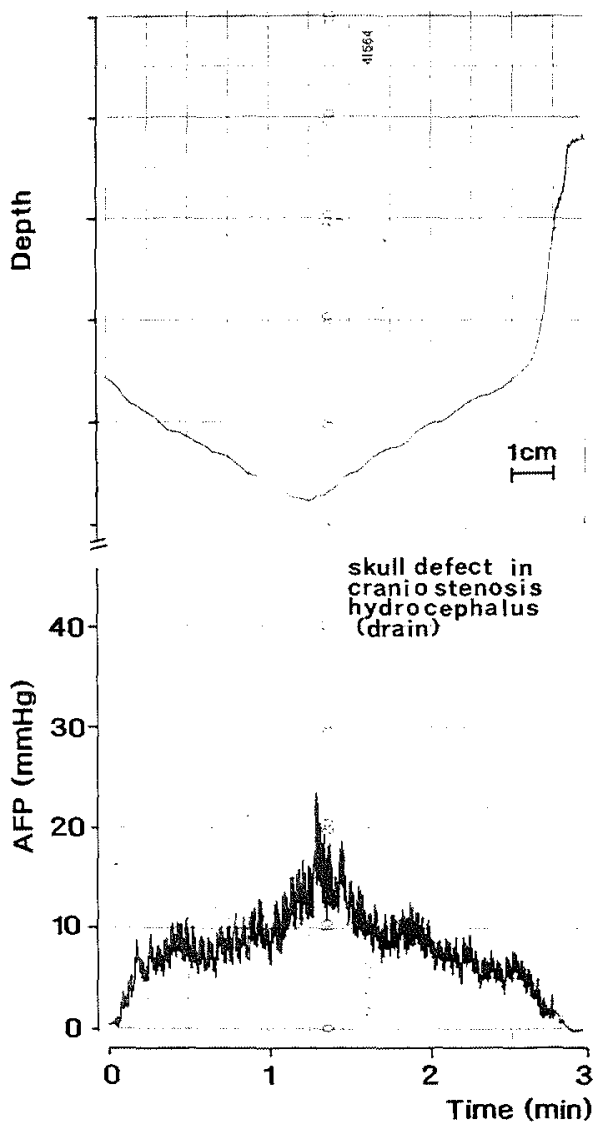

Fig. 1 Part of the short term AFP recording on patient 136, a boy of 8 years with multiple skull defects (operated craniosynostosis) and a shunt for hydrocephalus treatment. Note the PDC performed with the skull adaptor on a skull defect.

Fig. 4 Part of the continuous recording on patient 99, who was comatose due to bilateral subdural haemorrhages. Note the high level of AFP $(35-55 \mathrm{mmHg})$ and the multiple high amplitude B-waves (5-15 $\mathrm{mmHg}$ ).

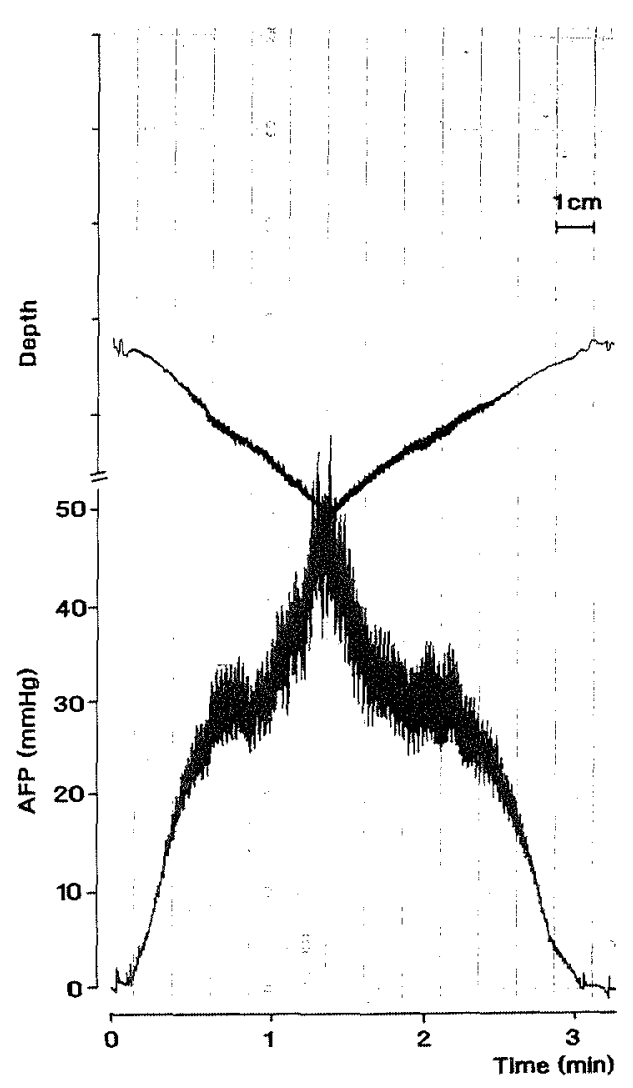

Fig. 3 Part of a short term AFP measurement performed in a patient with diagnosis triventricular hydrocephalus of unknown cause (patient 90).

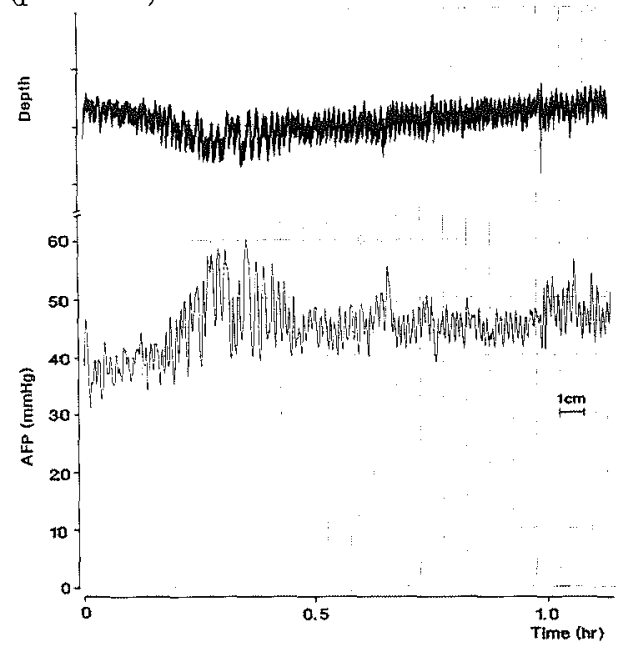




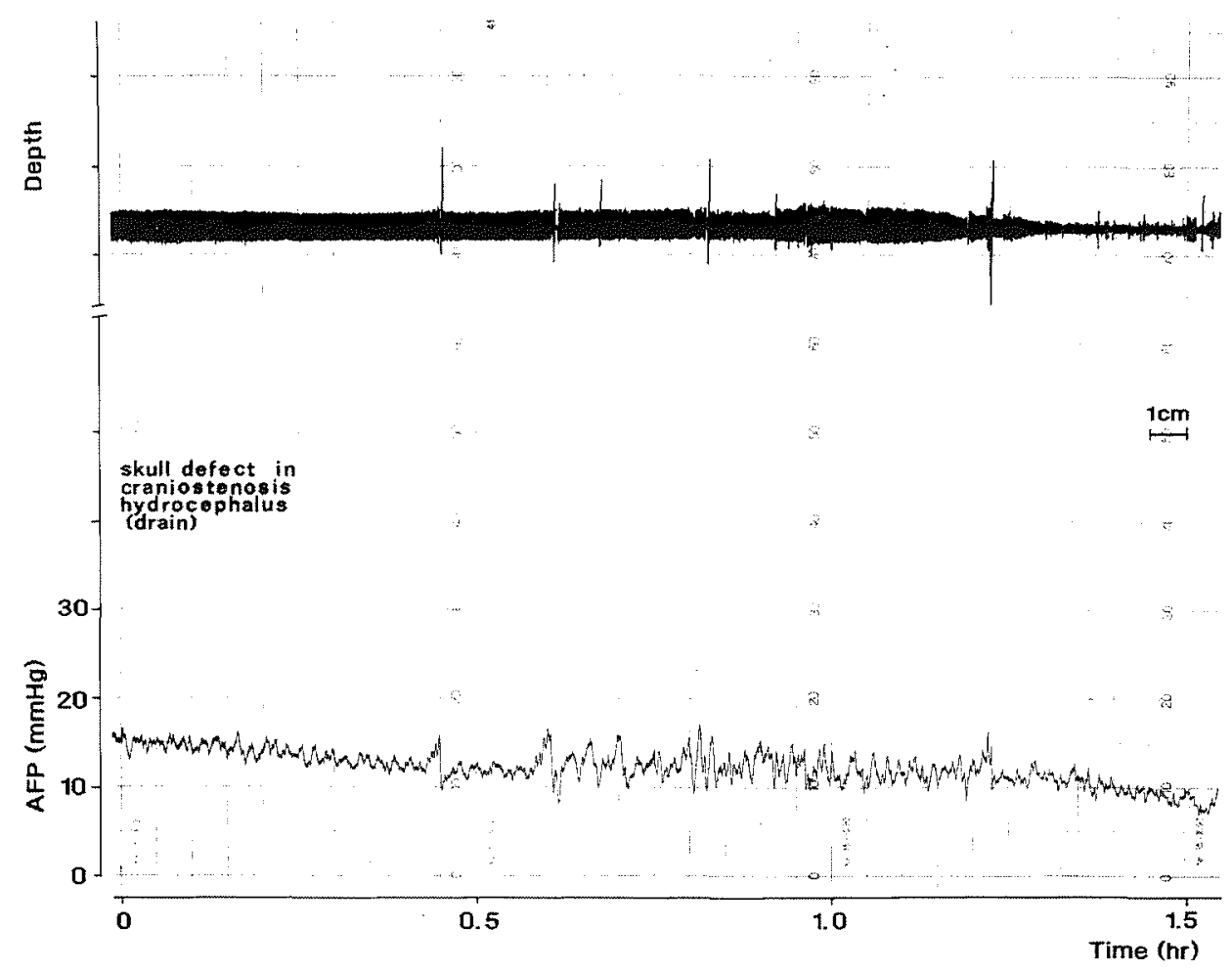

Fig. 2 Part of the continuous AFP recording performed on the same patient as in figure 1.

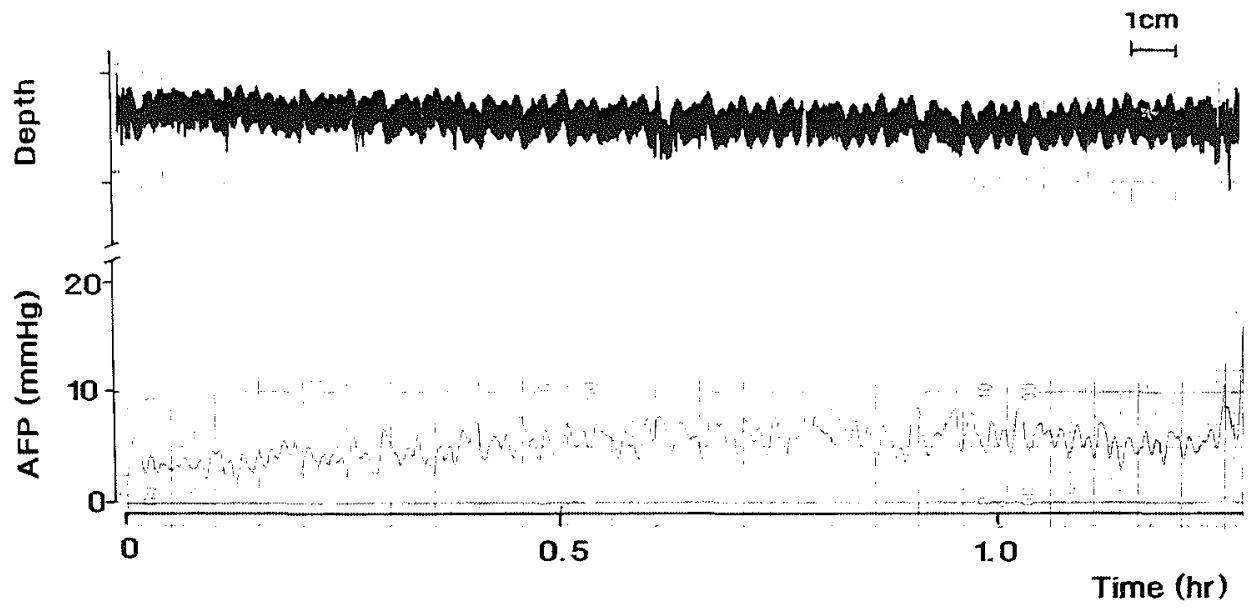

Fig. 5 Part of the continuous AFP recording on patient 73, who was comatose due to haemorrhagic shock encephalopathy. Note the average level of AFP $(5 \mathrm{mmHg})$ and the $B$-waves with an average amplitude of $5 \mathrm{mmHg}$. 


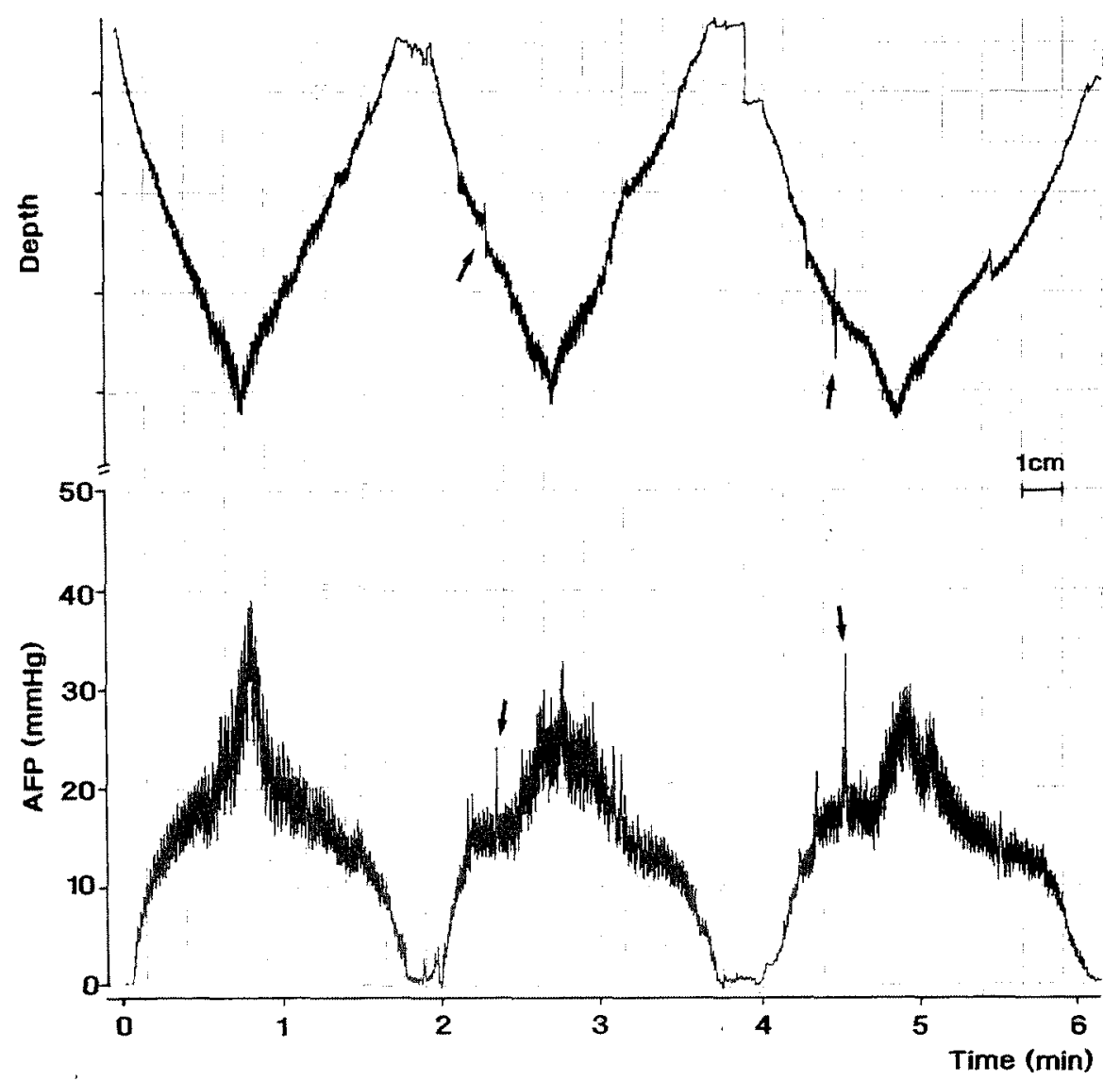

Fig. 6 PDC's in part of a short term AFP recording on patient 128. This child had multiple short lasting seizures. Arrows mark the movement artifacts, clearly visible at the same moment in both the AFP and the depth channel recording.

Figure 5 shows a part of the continuous recording in the patient with haemorrhagic shock encephalopathy (patient 73).

It is important to distinguish B-waves from movement artifacts. In the recordings we could read the movement artifacts from the depth registration channel because the depth channel showed saccadic alterations at the time of the movements due to a short change in the antenna position of the recording probe. In figures 6 and 7 examples of movement artifacts in a short term and continuous AFP recording are shown. Both recordings were done on a child with short lasting seizures (patient 128). When compared with the registration of the $\mathrm{B}$-waves (figures 4 and 5) a difference can be seen.

In the NCNS diagnostic classes B-waves were detected in four recordings in two patients (67 and 115). The percentages of registered B-waves were 32.0 in patient 67 with diaphragmatic hernia and 58.3 in patient 115 with tetralogy of Fallot. The amplitudes of the B-waves were $2.9 \mathrm{mmHg}$ in patient 67 and $5.3 \mathrm{mmHg}$ in patient 115. 


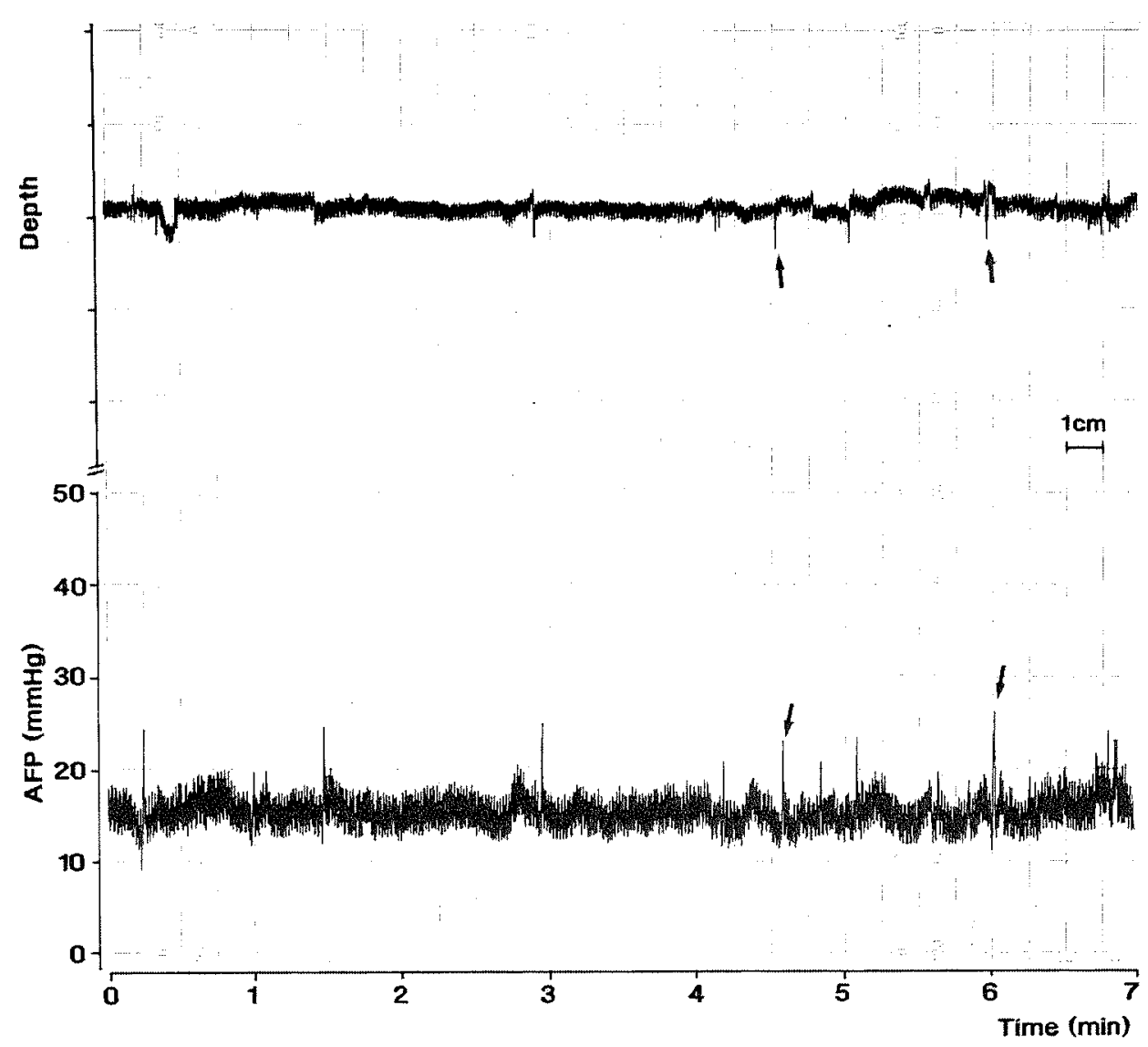

Fig. 7 Part of the continuous AFP recording on the same patient (patient 128). Arrows mark some of the movement artifacts, visible both in the AFP and the depth channel recording.

A-waves were captured in 10 of the continuous AFP recordings, performed on nine patients (eight patients with a CNS diagnosis and one patient with a NCNS diagnosis). In Table $\mathrm{V}$ the average baseline pressure and peak pressure of the $\mathrm{A}$-waves are given. Additional items such as the AFP level in the short term and in the continuous recordings, the duration of the interpretable recording time, the percentage of the recorded B-waves, the amplitude of the B-waves and any shunting operations performed are represented.

The $\mathrm{A}$-waves with peak pressure levels of
$65.4,64,59.8$ and $57.8 \mathrm{mmHg}$ were accompanied by AFP levels above 17.4 $\mathrm{mmHg}$ during the continuous recording, and more than $20 \%$ B-waves during the recording with an amplitude of more than $3.7 \mathrm{mmHg}$. In these patients a shunting operation was performed. Figures 8 and 9 show typical $\mathrm{A}$-waves occurring in the continuous AFP recording performed in patients 64 and 86.

The history of patient 67 will be described in chapter 7. The child with myelodysplasia (patient 69) had a sacral myelo- 


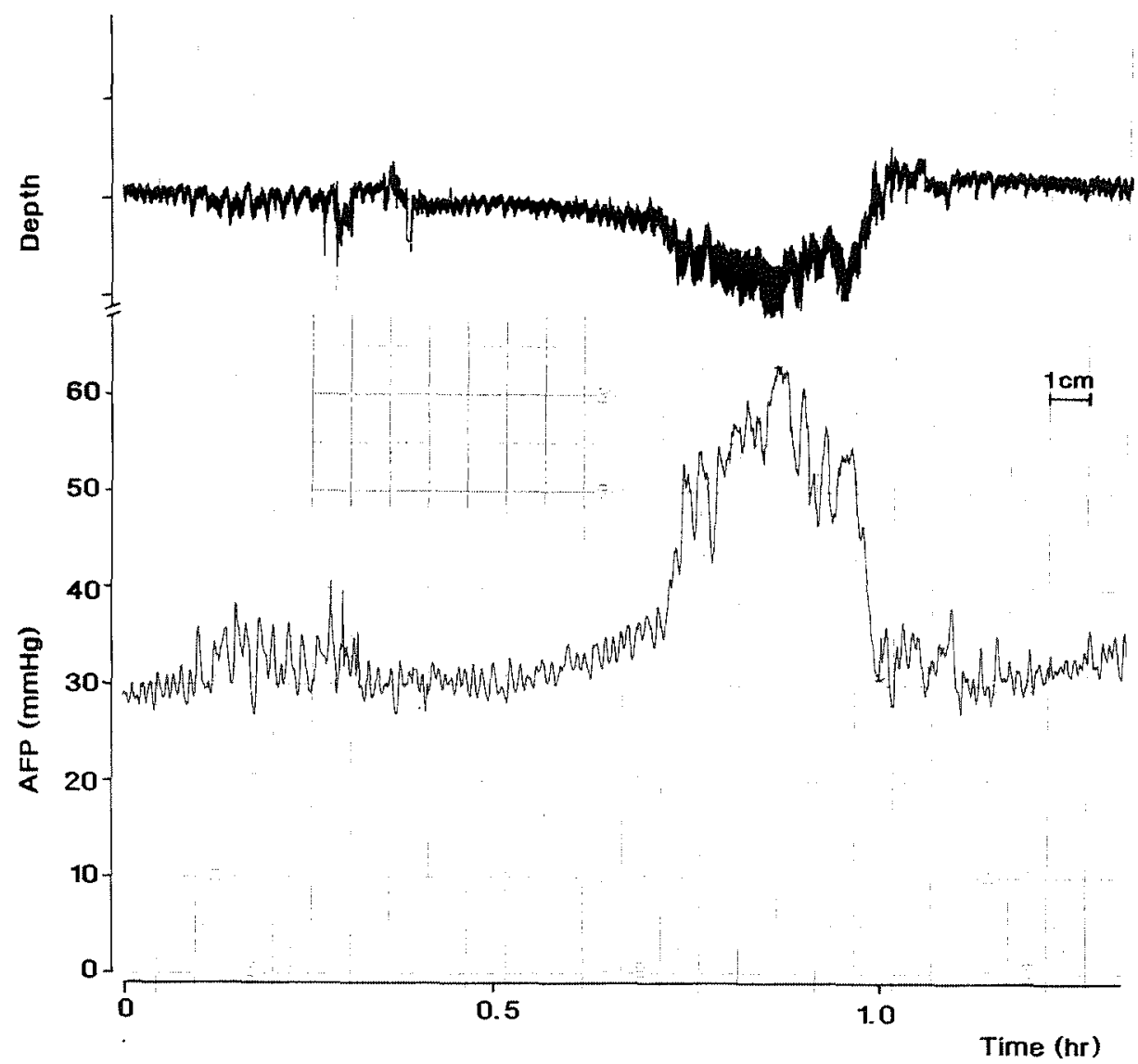

Fig. 8 Part of the continuous AFP recording on patient 64 showing a typical A-wave with a baseline pressure of $20.8 \mathrm{mmHg}$ and a peak level pressure of $64 \mathrm{mmHg}$.

meningocele with mild neurological signs, a slightly enlarged skull circumference and a normal psychomotor development up to this date. The levels of AFP in a total of 6 recordings performed during the first year of life decreased from 19.0 to 11.9 mmHg. No shunting operation was performed. Patient 86 with Gorlin's disease (Gorlin 1987) had clinical signs of raised ICP and slowly progressive hydrocephalus on sequential CT scans.

\section{AFP measurements before and after shunting operations AFP monitoring with the RTT was tested}

on 23 patients in whom 26 shunting operations or revisions were performed. The results of the 52 AFP measurements before and after the operation are shown in figure 10.

The average AFP level of the recordings before operation was $23.0 \pm \mathrm{SD} 7.9 \mathrm{mmHg}$ and $12.7 \pm \mathrm{SD} 4.0 \mathrm{mmHg}$ after operation. The difference was statistically significant (Wilcoxon $\mathrm{p}<0.01$ ).

In figure 11 an example is shown of a short term pre- and postoperative AFP measurement on a patient with diagnosis myelodysplasia (patient 72). 


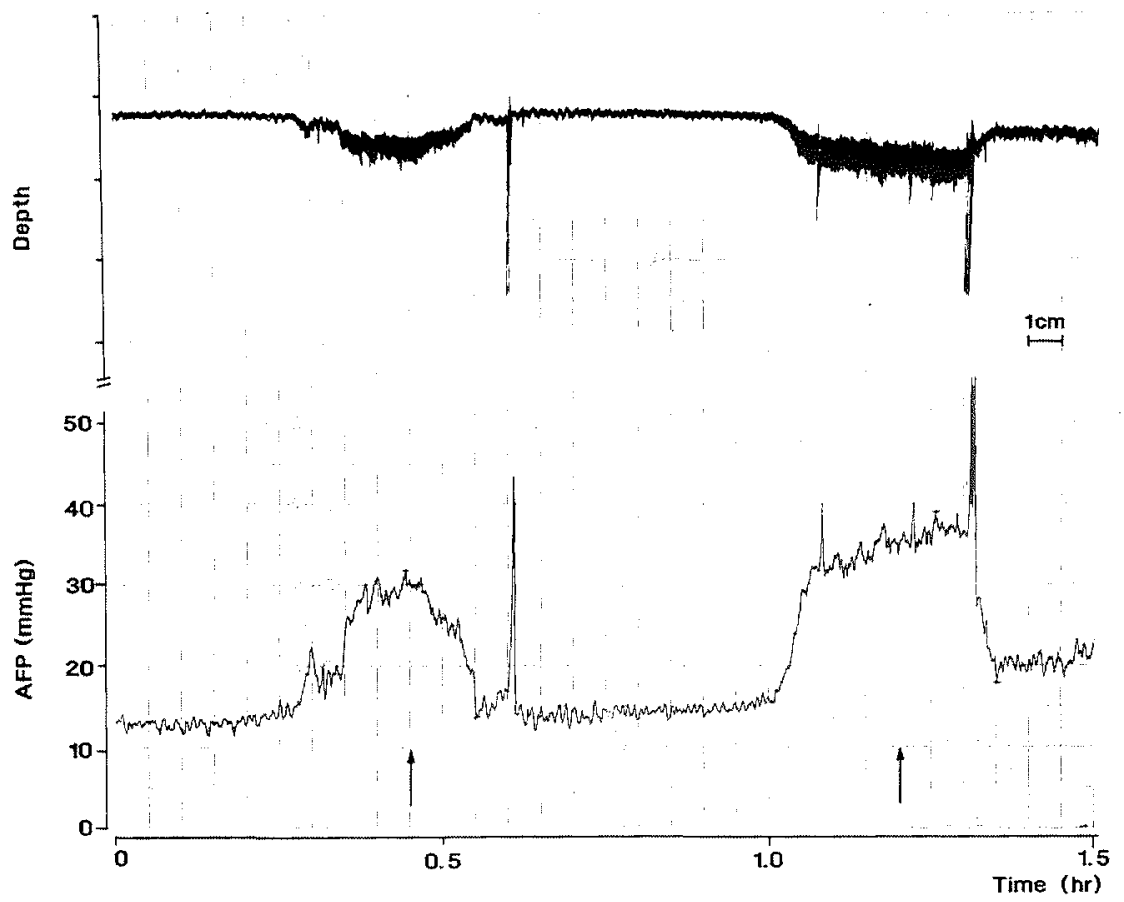

Fig. 9 Part of the continuous AFP recording on patient 86 showing two A-waves (arrows) occuring within one hour. The baseline pressures of the waves (arrows) are 13.8 and $17.8 \mathrm{mmHg}$, the peak level pressures are 31.6 and $38.9 \mathrm{mmHg}$.

\section{Comparative measurements} The results of the 16 comparative measurements between AFP and CSFP performed in 13 patients are represented in Table VI. Patients, type of comparative measurement, values of CSFP and AFP, including the differences are shown. The values are ranked according to the level of AFP.

The thirteen patients were all part of the CNS diagnostic classes. Seven patients (patients 12,95,99,105,108,121 and 147) belonged to the (cardio)vascular disorders and haemorrhages class of which six patients were diagnosed as perinatal haemorrhages and one patient (patient 12) as plexus papilloma. One patient (patient 146) had bacterial meningitis. Five patients belonged to the congenital and hereditary disorders diagnostic class of which two patients were diagnosed as macrocephaly and external hydrocephalus (patients 83 and 108), one patient was diagnosed as familial macrocephaly (patient 80), as macrocephaly and internal hydrocephalus (patient 36) and one as aquaductal stenosis (patient 120).

Figure 12 shows part of the recording of a comparative measurement with a closed manometer between AFP and CSFP (lumbar puncture) demonstrating the good relationship between the levels of AFP and CSFP.

Figure 13 shows part of the recording of a comparative measurement with a closed manometer between AFP and VFP. During this measurement, respiration was monitored. Note the good relationship 
Patient Diagnosis

code

\begin{tabular}{|c|c|c|c|}
\hline 134 & $\begin{array}{l}\text { macrocephaly + } \\
\text { internal hydro- } \\
\text { cephalus }\end{array}$ & 20.8 & 14.0 \\
\hline 90 & $\begin{array}{l}\text { triventricular } \\
\text { hydrocephalus of } \\
\text { unknown cause }\end{array}$ & 27.9 & 22.6 \\
\hline 71 & $\begin{array}{l}\text { quadriventricular } \\
\text { hydrocephalus of } \\
\text { unknown cause }\end{array}$ & 29.7 & 25.6 \\
\hline $86^{*}$ & $\begin{array}{l}\text { Gorlin's disease } \\
\text { and hydrocephalus }\end{array}$ & 30.4 & 17.3 \\
\hline $99 * \%$ & $\begin{array}{l}\text { bilateral subdural } \\
\text { haematoma }\end{array}$ & 39.3 & 32.0 \\
\hline $74 * 2 \times 2 *$ & $\begin{array}{l}\text { subependymal } \\
\text { haemorrhage } \\
\text { grade III }\end{array}$ & 24.3 & 18.0 \\
\hline $64 \div * * * 6$ & $\begin{array}{l}\text { intraventricular } \\
\text { haemorrhage grade } \\
\text { III }\end{array}$ & 19.5 & 17.4 \\
\hline $69^{* * * *}$ & myelodysplasia & 19.0 & 18.4 \\
\hline 67 & $\begin{array}{l}\text { diaphragmatic } \\
\text { hernia }\end{array}$ & 13.4 & 12.9 \\
\hline
\end{tabular}

AFP AFPc Interpretable B-wave B-wave A-wave A-wave Shunt $(\mathrm{mmHg})(\mathrm{mmHg})$ recording time \% during amplitude base line peak line yes/no (hours.minutes) recording $(\mathrm{mmHg})$ pressure $(\mathrm{mmHg})$

$12.5 \quad 25.8 \quad$ yes


TABLE VI: Sixteen comparative measurements between AFP and CSFP in thirteen patients. Patients, diagnosis, type of measurement, values of AFP and CSFP and pressure differences are given, ranked according to measured AFP level.

\begin{tabular}{|c|c|c|c|c|}
\hline \multirow{2}{*}{$\begin{array}{l}\text { Patient } \\
\text { code }\end{array}$} & \multirow[t]{2}{*}{ Diagnosis } & \multirow{2}{*}{$\begin{array}{c}\text { AFP } \\
(\mathrm{mmHg})\end{array}$} & $\mathrm{CSFP}(\mathrm{mmHg})$ & \multirow{2}{*}{$\begin{array}{l}\text { Difference } \\
\text { AFP - CSFP } \\
(\mathrm{mmHg})\end{array}$} \\
\hline & & & Open end manometer & \\
\hline 99 & $\begin{array}{l}\text { subdural } \\
\text { haematoma }\end{array}$ & 39.3 & 22.1 & 17.2 \\
\hline 146 & $\begin{array}{l}\text { bacterial } \\
\text { meningitis }\end{array}$ & 34.9 & 22.8 & 12.1 \\
\hline 113 & myelodysplasia & 25.7 & 14.0 & 11.7 \\
\hline 12 & $\begin{array}{l}\text { plexus- } \\
\text { papilloma }\end{array}$ & 23.6 & 13.2 & 10.4 \\
\hline 108 & $\begin{array}{l}\text { macrocephaly } \\
\text { and external } \\
\text { hydrocephalus }\end{array}$ & 23.3 & 9.6 & -13.7 \\
\hline 121 & IVH grade IV & 17.2 & 12.5 & 4.7 \\
\hline 105 & IVH grade III & 16.5 & 10.2 & 6.3 \\
\hline 95 & $\begin{array}{l}\text { intracerebral } \\
\text { bleeding }\end{array}$ & 13.4 & 9.6 & 3.8 \\
\hline 36 & $\begin{array}{l}\text { macrocephaly } \\
\text { and internal } \\
\text { hydrocephalus }\end{array}$ & 10.7 & 8.1 & 2.6 \\
\hline 99 & see above & 6.4 & 5.9 & 0.5 \\
\hline 99 & see above & 6.1 & 5.5 & 0.6 \\
\hline \multirow[t]{2}{*}{120} & \multirow{2}{*}{$\begin{array}{l}\text { aquaductal } \\
\text { stenosis }\end{array}$} & \multirow[t]{2}{*}{5.5} & 5.2 & \multirow[t]{2}{*}{0.3} \\
\hline & & & Closed type manometer & \\
\hline 99 & see above & 21.3 & 22.3 & -1.0 \\
\hline 83 & $\begin{array}{l}\text { macrocephaly } \\
\text { and external } \\
\text { hydrocephalus }\end{array}$ & 14.6 & 12.9 & 1.7 \\
\hline 147 & IVH grade III & 12.2 & 8.8 & 3.4 \\
\hline 80 & $\begin{array}{l}\text { familial } \\
\text { macrocephaly }\end{array}$ & 8.5 & 7.9 & 0.6 \\
\hline
\end{tabular}

\section{Table V}

* The parents of this patient refused a shunting operation.

* In patient 99 two continuous AFP recordings were performed.

The average AFP values of these two recordings are given.

:*:*: In this patient the level of $A F P$ was increased in the first recording.

In the first year of life the AFP level decreased (see text).

Subependymal baemorrbage grade III and intraventricular baemorrbage grade III represent blood within the ventricles accompanied by ventricular dilatation (Papile et al. 1978). 
between the levels of AFP and VFP, and also the recorded A-wave.

\section{DISCUSSION}

CNS and NCNS diagnostic classes

The AFP values in the heterogeneous group of patients were shown in Tables III and IV. Most values found were as expected on the basis of the clinical signs. The CNS and NCNS diagnostic classes will be discussed separately.

In the CNS diagnostic classes all children with hydrocephalus had AFP values above $15 \mathrm{mmHg}$. This is above the level of normal AFP $11.0 \pm 2.9 \mathrm{mmHg}$ as measured on children aged 30 days or more (chapter 4).

The children with meningitis had increased pressures probably due to a block of CSF circulation, oedema or hydrocephalus. Increased ICP such as we found in patients with bacterial meningitis was also reported by Devictor et al. (1983), Goitein et al. (1983) and Minns and Engleman (1988).

The children with AV malformation, choroid plexus papilloma and Sturge Weber had hydrocephalus with clinical signs of increased ICP.

Among the children with perinatal haemorrhages were both premature and term born infants. Most of those children will develop hydrocephalus sooner or later (Dykes et al. 1989, Volpe 1989a). This was also found in our patients. To monitor this a few patients were followed as outpatients for control AFP measurements. The patient with haemorrhagic shock encephalopathy was comatose and on artificial ventilation. The AFP level remained below $10 \mathrm{mmHg}$. During the continuous AFP recording there was no gross elevation of pressure, however there were $5.4 \%$ $\mathrm{B}$-waves, no $\mathrm{A}$-waves. $\mathrm{B}$-waves in patients

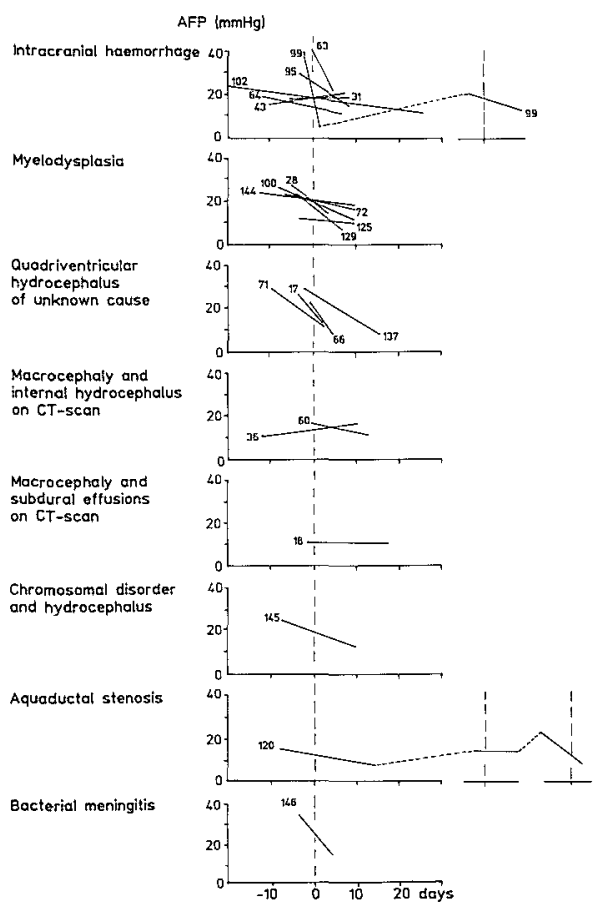

Fig. 10 Effect of a shunting or shunt revising operation on the level of ICP in 23 patients. Pre- and postoperative AFP values $(\mathrm{mmHg})$ of 26 operations are represented, according to the time of the pre- and postoperative measurements (days). The patients are identified by their number. The vertical broken lines represent the time of operation.

treated with artificial ventilation were also reported by Lundberg (1960).

Among the craniosynostosis patients were two patients with stenosis of the sagittal sutures, nine patients had either Crouzon, Apert or Pfeiffer syndromes in which stenosis of more than two sutures exists. In these latter patients hydrocephalus is often already present at birth or arises soon after birth. Furthermore there is some evidence that impaired venous outflow may cause increased ICP and/or hydrocephalus (Shulman and Ransohoff 1965, Kinal 1962, De Lange and De Vlieger 1970, Rosman 

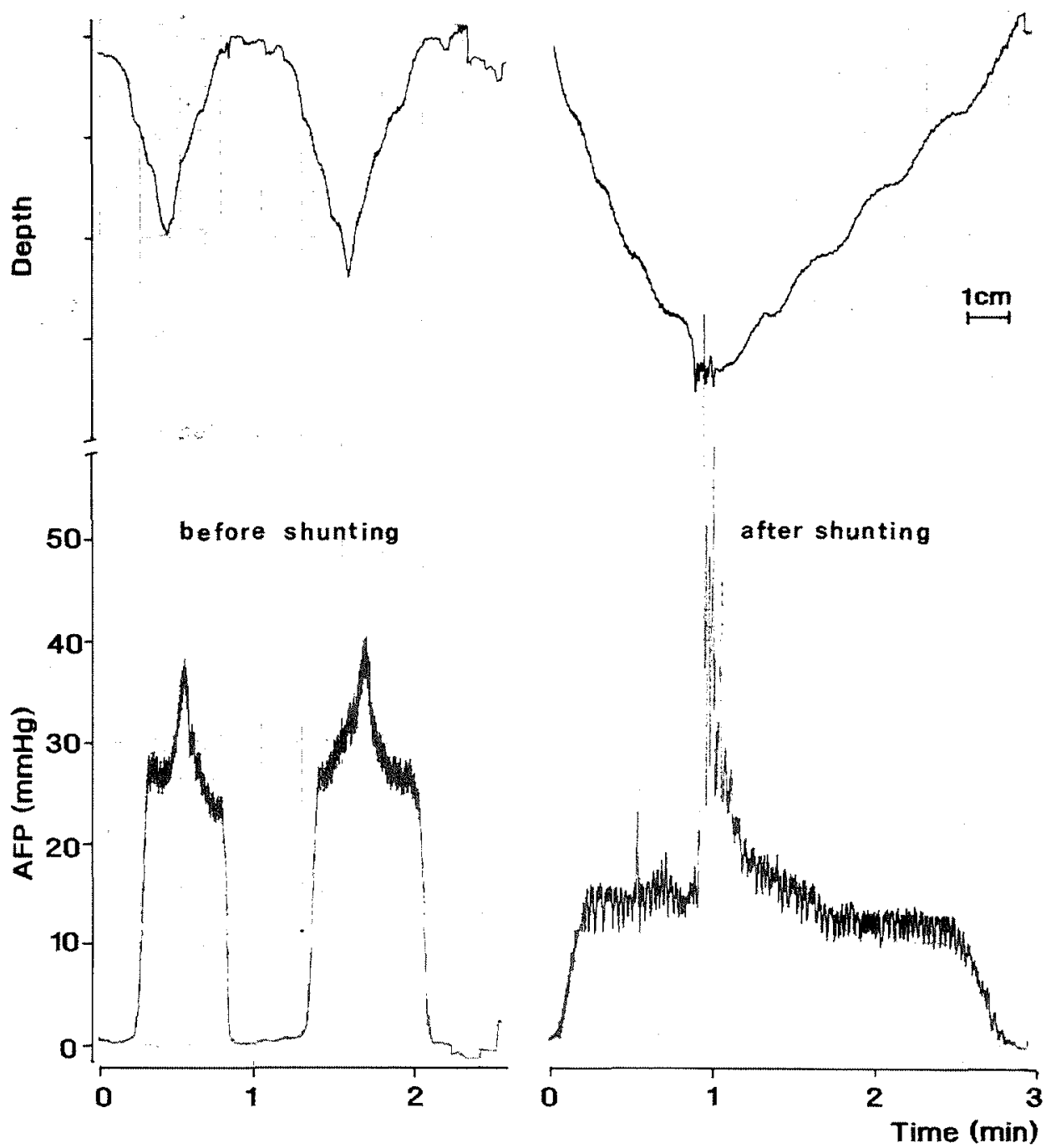

Fig. 11 Parts of a short term pre- and postoperative AFP measurement performed on patient 72 (diagnosis myelodysplasia).

and Shands 1978, Young 1979, Sainte Rose et al. 1984, Steinbok et al. 1989). The mean AFP level of these eleven patients is clearly raised compared to the normal AFP values (see chapter 4). Due to the small number of patients it was not possible to investigate any differences in AFP values between infants with stenosis of one suture and more than two sutures. This increase in AFP level in craniosynostosis is in agreement with the literature (Renier et al. 1982, Golabi et al. 1987). Of the 20 patients with myelodysplasia 19 had clinical signs of hydrocephalus, confirmed by CT-scan.

The patients with congenital disorders like agenesis of corpus callosum, arachnoid cysts, aquaductal stenosis, Dandy Walker 


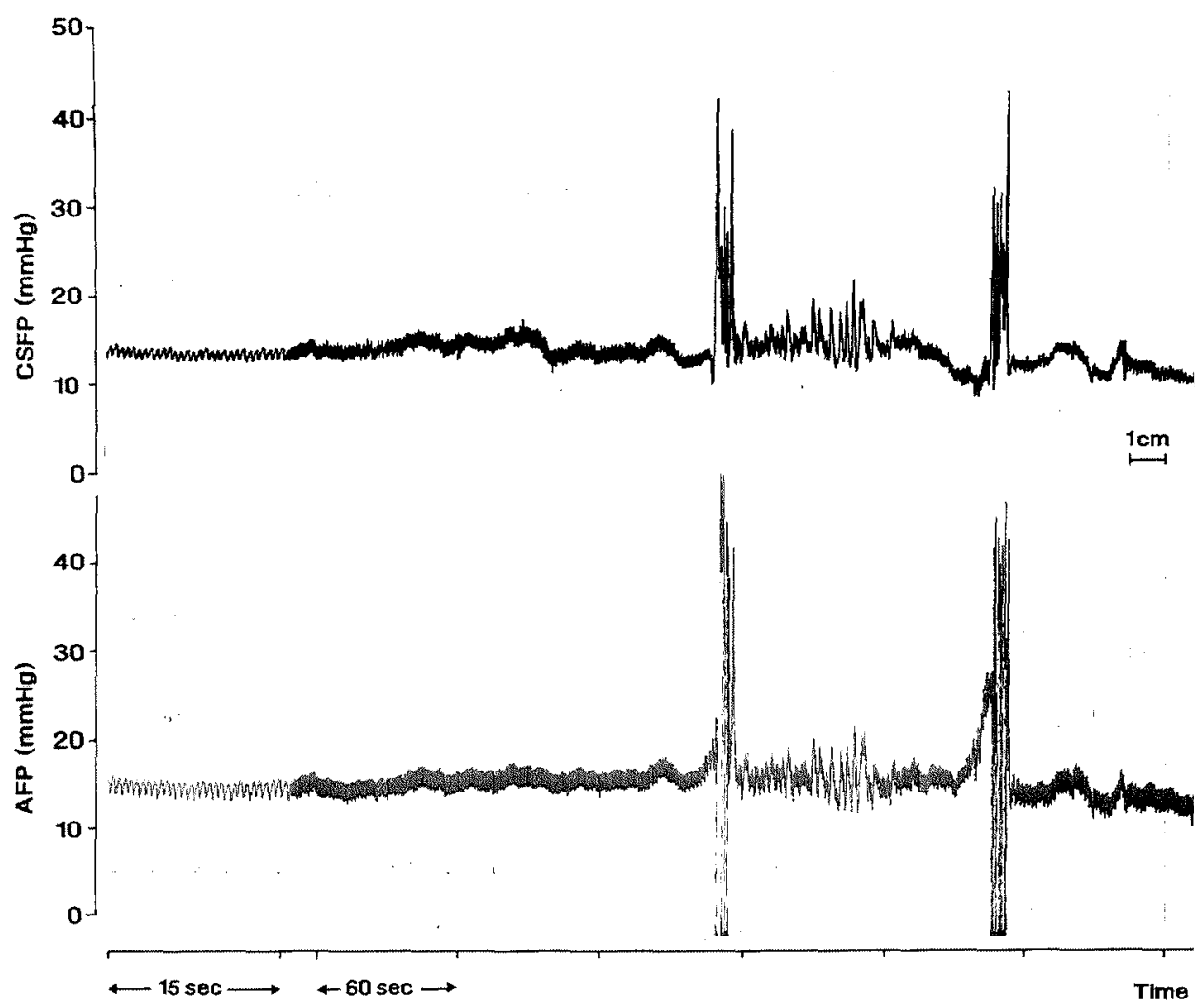

Fig. 12 Part of the recording of a closed type comparative measurement between AFP and CSFP (lumbar puncture).

malformation and oculomotor apraxia did not have clinical signs of increased ICP during the AFP measurement. The recorded pressures were in the normal range, as was expected.

The children with leucodystrophy, neuroblastoma and psychomotor retardation did not have clinical signs of increased ICP and a normal AFP was recorded.

The patients with porencephalic cysts had clinical signs of hydrocephalus, the recorded pressures are above normal.

The infants with clinical signs of elevated ICP in whom tri- or quadriventricular hydrocephalus of unknown cause was diagnosed with CT-scan or ultrasound, all had AFP levels above $15 \mathrm{mmHg}$.
The infants with macrocephaly without any focal neurological symptoms were divided, according to the CT-scan results, into those with macrocephaly and internal or external hydrocephalus, subdural effusions or no abnormalities on the CTscan (see also chapter 7). Macrocephalic children still form a group of children for discussion. At the very beginning of this fontanelle research project we measured AFP only in macrocephalic children. However, because of the relatively small number of children with this diagnosis in our clinic we changed the study design into a more general study on the AFP measuring technique. Due to this initial interest the group of macrocephaly will be 

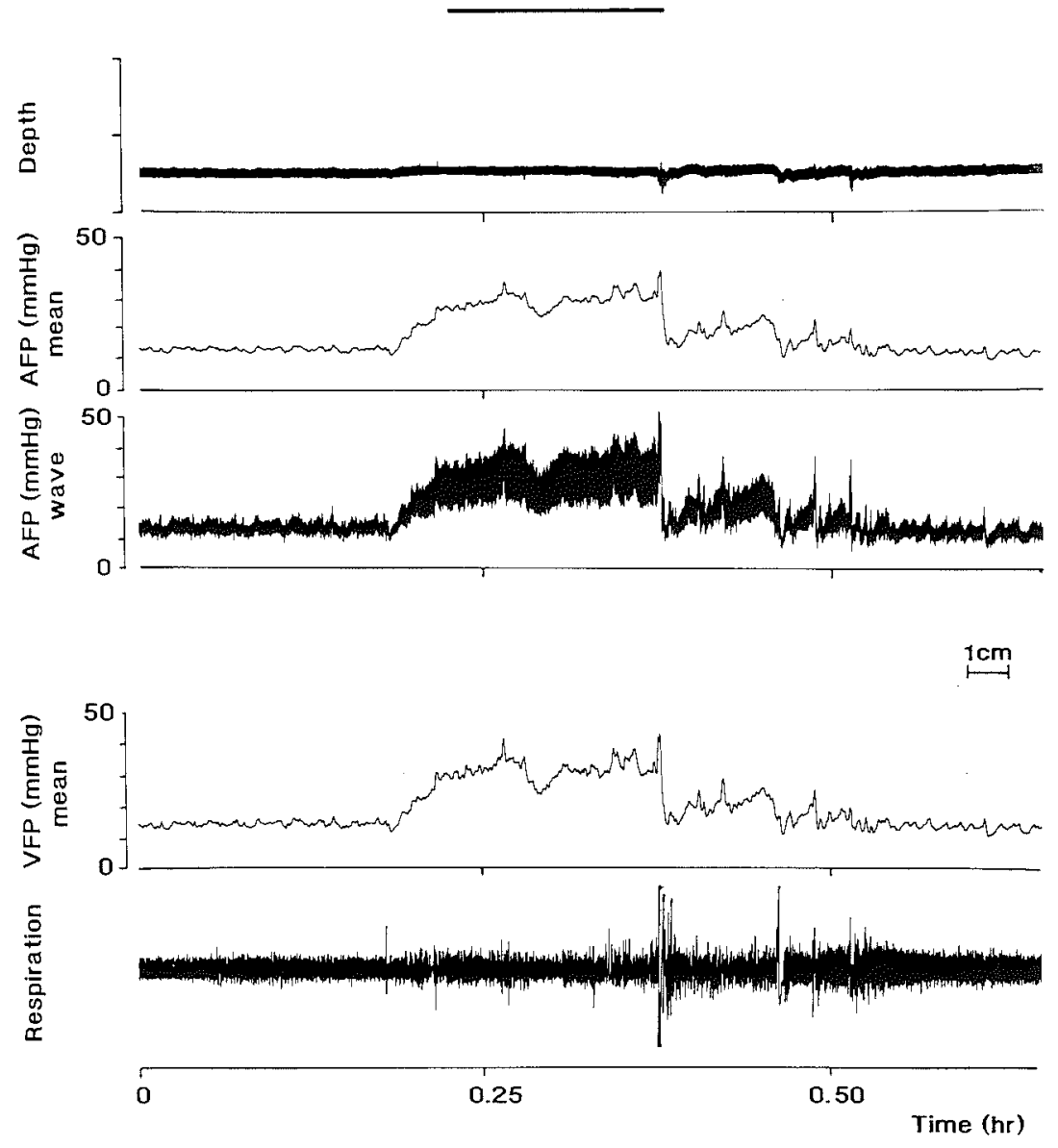

Fig. 13 Part of the recording of a closed type comparative measurement between AFP and VFP. The A-wave with a duration of about 20 minutes is clearly visible.

discussed somewhat more extensively than the other groups mentioned.

Macrocephaly or a large head applies to any head with an excessive occipito-frontal circumference (exceeding the mean by more than two standard deviations) without regard to cause or brain size (Nellhaus 1968, DeMyer 1972, 1986, Weaver and Christian 1980, Lorber and Priestley 1981). The most common causes of macrocephaly are: obstructive hydrocephalus, communicating hydrocephalus divided into internal or external hydrocephalus, brain oedema, subdural fluid collections, thickened skull and megalencephaly (oversized and overweight brain), subdivided into metabolic and anatomic megalencephaly. As it is beyond the scope of this thesis to discuss extensively the many causes of macrocephaly the reader is referred to an excellent review published by DeMyer (1986). On megalencephaly and CSF dynamic studies work has been published by Bresnan and Lorenzo (1975), Portnoy and Croissant (1978), Gooskens et al. (1985), and Gooskens (1988). 'Communicating hydrocephalus' has been the subject of a large number of publications. Various definitions have been used such as benign communicating hydrocephalus (Kendall and Holland 1981), external hydrocephalus (Robertson and Gomez 
1978, Alvarez et al. 1986), benign enlargement of the subarachnoid spaces (Sahar 1978, Ment et al. 1981), benign subdural collections, subdural effusions and hypodense extracerebral fluid collections (Naidich et al. 1976, Robertson et al. 1979, Rothenberger and Brandl 1980a,b, Modic et al. 1981, Clar et al. 1981). The overall opinion is that in most children the syndrome has a benign self-limiting course (Nickel and Galbenstein 1987) and no invasive interventions are necessary. Shunting procedures should be considered only in the case of clinical progression. In our patients we saw a higher level of AFP in the children with internal and external hydrocephalus on CT-scan than in the children with subdural effusions on CTscan and the ones with familial macrocephaly. The numbers however are small and no conclusions can be drawn.

The child with head injury and skull fractures was comatose. The high level of AFP was due to oedema and multiple small intracerebral haemorrhages (established by CT-scan). During the continuous AFP monitoring the treatment of raised ICP with ICP lowering agents was monitored.

In the NCNS diagnostic classes we expected to measure 'normal' AFP levels. However, in some of the patients, elevated levels of AFP were found. In the patients with cardiac disorders, this could be caused by increased venous pressure in the intracranial compartment (Shulman and Ransohoff 1965, Norrel et al. 1969, Silver et al. 1970, Rosman and Shands 1978, SainteRose et al. 1984).

The patients with omphalocele, oesophageal atresia and sickle cell disease were sucking and pushing to empty their bowels during the recording. These Valsalva manoeuvres are known to cause an increase in ICP.

In the child with osteogenesis imperfecta the high level of AFP was an unexpected finding. However, it has been reported that in osteogenesis imperfecta macrocephaly can occur, as was the case in this infant. The high level of ICP could be caused by venous outflow impairment due to basilar impression as has been reported by Pozo et al. (1984) and Tsipouras et al. (1986).

\section{Continuous measurements}

The purpose of continuous monitoring of intracranial pressure both in adults and in children is to investigate the level of pressure. Furthermore the relationship between pressure and body position, state of consciousness or physiological variations like respiration and arterial pulse wave, can be determined (Lundberg 1960, Hayden et al. 1970, Paraicz and Vajda 1975a,b, Magnaes 1976a,b, Welch 1980, Perlman and Volpe 1983, Miller 1985).

Lundberg (1960) defined at least two typical ICP waves. A-waves, or plateau waves, are due to cerebral vaso-instability (Risberg et al. 1969, Arnold and Laas 1980) and suggest poor intracranial compliance, a condition in which small additions of volume to the intracranial contents result in large increments of pressure (Rosner and Becker 1983, Miller 1985). In AFP monitoring A-waves could also be detected, however the shape of these waves was slightly different from A-waves described in adult parients. This has also been reported by others (Paraicz 1978, 1982). The shape of an A-wave was reported to be rather like a hill or dome instead of the plateau seen in adults and the amplitude seemed to be related to age. In younger children the amplitude was lower. Paraicz (1982) stated that this might be caused by the greater expandibility of the infant's skull.

Another wave pattern was called B-waves. 
$\mathrm{B}$-waves are also considered to be of vascular origin but are quite different in shape, consisting of sharply peaked waves occurring once every one or two minutes and often coinciding with phasic alterations in respiration (Lundberg 1960, Auer and Sayama 1983, Venes 1979). Bwaves tend to occur in patients with disturbed consciousness, or with raised ICP and are usually accompanied by periodic breathing (Lundberg 1960).

B-waves were detected in a heterogeneous group of patients. Usually the respiration was not recorded so we could not relate $\mathrm{B}$-waves to alterations in respiration patterns. The amplitude of the B-waves tends to increase with a rise in ICP (see figures 4 and 5), according to the V-P concept (Avezaat and Van Eijndhoven 1984).

A third wave type, C-waves, was also defined by Lundberg. These $\mathrm{C}$-waves are oscillations occurring at a frequency of about 4 to 8 per minute with varying amplitude. Lundberg (1960) did not consider these waves of much clinical importance. In the AFP recordings in this study no $\mathrm{C}$-waves were observed.

\section{Pre- and postoperative measurements}

Pre-operative AFP measurements on infants with clinical signs of increased ICP due to hydrocephalus showed high levels of AFP. As was expected, a significant decrease in AFP was found after a shunt operation. Increased ICP was also detected in a few children some time after the operation. Although they had only minor clinical signs of drain dysfunction the pressures were high. Drain dysfunction was corrected and after a second or third operation the AFP was significant lower than before the operation. In children with an open fontanelle AFP monitoring there- fore is a useful and a harmless method of checking the function of a shunt.

\section{Comparative measurements}

Non-invasive ICP measuring techniques are preferable to the standard method of ventricular fluid pressure measurement. In children, comparison of AFP and VFP requires the performance of invasive procedures. Therefore, on ethical grounds, invasive recordings were only performed if there existed a clinical indication for performing lumbar or fontanelle puncture, for instance to examine the CSF, or to vent CSF temporarily in case of obstruction hydrocephalus in patients with intracranial haemorrhages. Although the number of comparative measurements is small and although both open end and closed manometers were used we can state that in the children with low pressures (below $10 \mathrm{mmHg}$ ), the difference between AFP and CSFP is less than one $\mathrm{mmHg}$. As one $\mathrm{mmHg}$ can be considered an acceptable measurement error we can state that the difference is not significant. AFP measurement therefore is as accurate as CSFP measurement with an open end manometer in the low pressure range. If the AFP was higher (above $15 \mathrm{mmHg}$ ) the difference between AFP and CSFP measured with an open end manometer was greater. This is easily understood, since in the range of higher pressures the compliance is lower and the craniospinal system is changing from the flat part to the steep part of the volume-pressure curve resulting in a greater change in pressure in non-isovolumetric measurement (Miller et al. 1973, Löfgren et al. 1973, Avezaat and Van Eijndhoven 1984). In figure 14 the volume-pressure curve, obtained in animal experiments, is shown (Löfgren et al. 1973).

The closed type comparative measurements showed a good relationship of AFP 
and CSFP with a maximum difference of $3.4 \mathrm{mmHg}$ and a minimal difference of 0.6 $\mathrm{mmHg}$. The mean difference is $1.9 \mathrm{mmHg}$ and can be considered of no clinical relevance. The closed type comparative measurements show that there is a good relationship between AFP measurement with the RTT and CSFP, however the number of observations is too small to draw any definite conclusions.

\section{General remarks}

From the results of this study it can be concluded that this method of anterior fontanelle pressure monitoring is a reliable and easily applicable technique for measuring ICP in young children. Short term AFP measurements can be done to determine the level of pressure, and this is important in the case of (developing) hydrocephalus for instance in prematures with intraventricular haemorrhages (Volpe 1989a,b). Furthermore the drain function can be checked after shunting operations. The technique proved to be reliable not only during the short term but even more so during the continuous AFP measurements. Not in a single case did the fixation frame break. Furthermore, if necessary, PDC's could always be easily repeated. This shows that during the total recording time the fontanelle adaptor was kept in place maintaining the RTT at accurate depth in the fontanelle.

Continuous ICP recording is usually done in neurosurgical or pediatric intensive care wards on patients with head-injuries, or on patients with intracranial mass lesions, before or after operation. The studies on continuous ICP recording usually concern invasive measurement techniques applied in children with hydrocephalus (Hayden et al. 1970, DiRocco et al. 1975, Portnoy and Croissant 1978, Shapiro et al. 1984), Reye's syndrome (Kindt et al. 1975, Mickell and Ward 1984), hypoxic-ischae-

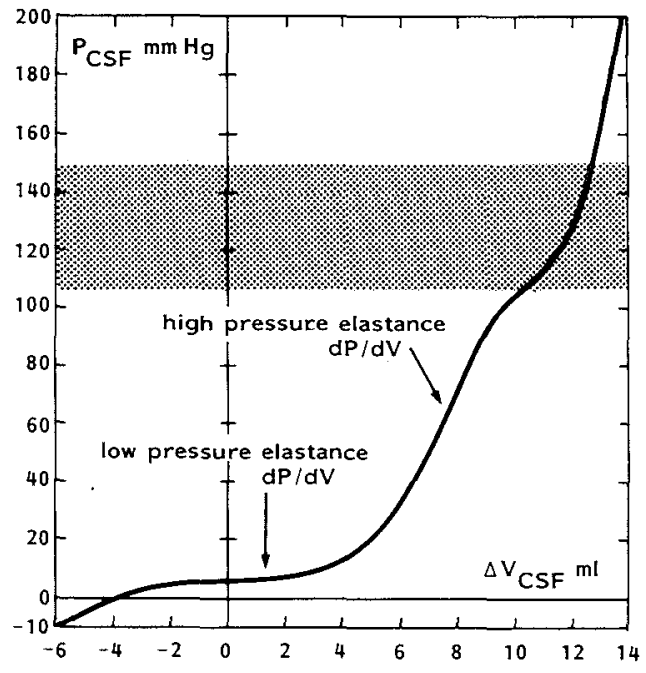

Fig. $14 \mathrm{CSF}$ volume-pressure curve obtained in the dog with rapid infusion of fluid $(0.25 \mathrm{ml} /$ sec) into the cisterna magna.

(Reproduced, with permission from, Löfgren et al. 1973, Acta Neurol Scand 49:557-574). Systolic and diastolic blood pressure are indicated by the shaded area. In clinical ICP range the curve can be characterized by two straightline segments: low pressure and high pressure elastance respectively.

mic encephalopathy secondary to cardiac arrest, strangulation or near- drowning (Donn and Philip 1978, Levene and Evans 1983, 1985, Levene et al. 1987, Raju et al. 1980, 1981, 1986, Fischer et al. 1982, Nussbaum and Galant 1983, Sarnaik et al. 1985), head injury (Bruce et al. 1976, 1979), postoperative measurement in craniostenosis (Sainte-Rose et al. 1980) and intracranial haemorrhages in term and preterm infants (Hill 1983, Dykes et al. 1989, Volpe 1989a,b). In all these studies monitoring of ICP was indicated to check the level of pressure, to detect any pathological changes and to guide the management efforts aimed at preventing secondary brain-injury (Mickell and Ward 1984). 
However invasive techniques all carry risks (see chapter 2) of which infection and bleeding are the most important. The results of the continuous AFP measurements found with this technique can be regarded as reliable. It was possible to measure the trend of ICP and specific wave patterns, like $\mathrm{A}$-waves and B-waves. Furthermore, in cases of macrocephaly when the base level pressure is slightly elevated, continuous AFP monitoring under controlled conditions (for instance combined with monitoring of respiration), could provide more insight into the existence of pathologic wave patterns. Most likely the combination of clinical examination, brain-imaging techniques, clinical course and also AFP monitoring is the most effective in deciding upon the question of whether or not to shunt.

\section{CONCLUSIONS}

1 In addition to clinical signs and ancillary examinations in patients with various neurological and non-neurological diseases, both short term and continuous measurement of ICP via the anterior fontanelle seem valuable parameters in deciding whether a child needs to be shunted or not.

2 Continuous AFP monitoring with the
RTT and fixation frame is feasible. Fluctuations in pressure and pathological wave patterns can be recorded.

3 This non-invasive method can check drain function in children if the fontanelle is still open.

4 The few comparative measurements with closed manometer showed a good relationship between AFP and CSFP. 


\section{INTER-OBSERVER ANALYSIS IN A RANDOM SAMPLE OF ANTERIOR FONTANELLE PRESSURE RECORDINGS}

\section{INTRODUCTION}

Every new recording method should be evaluated for accuracy. From a technical point of view the calibration of a recording system should be precise and the recorded values should represent true values. Interpretations of the recordings should be standardized in order to prevent observations varying among various interpreters. The variations in observer interpretation can be divided into systematic variation, which is the inter-observer variation as fixed effects, and the nonsystematic (random) variation. This random variation is caused by other error sources of interpretation including individual-observer (intra-observer) variation. It is relevant to distinguish between these two variations (systematic and non-systematic) since a large random variation could mask a systematic variation.

The procedure of measuring AFP with the Rotterdam Teletransducer (RTT) consists of a standard series of steps (see chapter 3). The fixation frame and the fontanelle adaptor are attached according to a standard procedure. First the PDC has to be recorded to establish the correct depth of the transducer for measuring AFP. This PDC can be reliably found and is easy to reproduce. The plateau in the PDC represents ICP. Depending on the stillness of the baby and on the fontanelle size it usually takes 2-5 minutes to record a single PDC (with an ascending and descending plateau). Since ICP varies with arterial pulsation, respiration and Valsalva maneuvers a few consecutive PDC's of good quality (i.e. with two legible plateaus) should be recorded to measure the average ICP over a certain period. We chose to take three consecutive PDC's, since in each recording of the patients there were at least three PDC's with discernable plateaus available. Furthermore a standardized method for measuring AFP from a recording is necessary in order to make general inferences about measurement results.

Each new technique usually works in the hands of its inventor. The first phase of testing new medical equipment usually concerns animal experiments (De Jong et al. 1979). The second phase concerns the testing of the new equipment by clinicians in a clinical setting. The third phase concerns the testing of the new equipment in another clinic. The fourth phase concerns the commercializing of the new equipment. The results of this study concern the second phase. During the first, and the beginning of the second phase of this study the measurements were performed by the inventor (DA de Jong). Thereafter all measurements were performed by the author of this thesis. 
In order to examine whether others (both clinicians and non-clinicians) are also capable of judging the level of AFP from a PDC in an AFP recording, the present study was undertaken. There are several reasons why it can be difficult to establish exactly the level of AFP. For instance, there can be spikes in the PDC's due to movement artifacts resulting in difficulties in judging the curves. Furthermore, in small fontanelles it is not always easy to perform a PDC, therefore the quality of the recorded PDC plateau might not be optimal.

It is expected that both the inventor and the author of this thesis are capable of judging the level of pressure from the PDC's in an AFP recording. If investigators with different backgrounds draw the same conclusion in judging the level of AFP from a recording than this recording method might prove useful in other hands and in clinical practice.

It is important to realize that in this study four sources of variation can be distinguished:

a Patient: the inter-patient variation.

b Observer: the inter-observer variation. c PDC: intra-patient (or biological) variation.

d Other variations: measurement error.

The intra-observer variation was not distinguished separately.

With a variance-components analysis it might be possible to estimate the influence of each variation source.

\section{The aims of this part of the study were}

1 To investigate the inter-observer variation in the interpretation of AFP recordings.

2 To investigate whether random variation is somehow related to the quality of the AFP recording and the quality of the
PDC plateaus as judged by the observers. 3 To investigate the influence of each of the four variation sources present in this study.

\section{MATERIAL AND METHODS}

Four observers independently evaluated a number of AFP recordings. These recordings were randomly selected both from the total group of normal newborns (group I) and infants (group II) and from the group of patients (group III). Of group I, five recordings of five patients were selected, of group II, 16 recordings of 10 patients and of group III, 61 recordings of 35 patients.

The four observers were given a codenumber:

1 The author of this thesis.

2 A physicist with special interest in intracranial pressure.

3 A child neurologist, not familiar with the technique of AFP monitoring.

4 A clinical neurosurgeon with interest in intracranial pressure.

First the author evaluated every recording. All PDC's with discernable plateaus were numbered, no other notes were present on the recording paper. To calculate the AFP value the procedure, as mentioned in the introduction of this chapter, was carried out. When this had been completed the 82 randomly selected AFP recordings were handed over to the other three observers in batches of 10 recordings each time. They assessed the AFP recordings by calculating the pressure values of the numbered PDC's by means of the ICP calculator (see chapter 4). Each observer was unaware of the AFP values calculated by the others.

The AFP value in a standard AFP recor- 
ding was called the AFP score, this being the average of the AFP values of the first three PDC's, where the AFP value of a single PDC was calculated as the average of the pressure of the ascending plateau $(\mathrm{Pa})$ and the descending plateau $(\mathrm{Pb})$. See chapter 4, figure 3 . It is difficult to generalize when in one patient the AFP value would be calculated as the average of three PDC's and in the other patient as the average of e.g. six or ten PDC's. Furthermore the variation of AFP did not decrease substantially when AFP was calculated as the average of maximally ten PDC's instead of three PDC's.

During the assessment of the recordings, the four observers judged the quality of the recording and of the PDC plateaus according to a subjective, ordinal 5 points scale: 1: excellent, 2: good, 3: fair, 4: moderate, 5: bad.

The Wilcoxon matched pairs signed rank test (a non-parametric test to test significance of difference between two paired data) tested the systematic (inter-observer) difference between each pair of observers as fixed effects.

The Spearman rank correlation (a nonparametric test to compare the ranking of two distinct observations, which are difficult to quantify) tested the other interpretation errors. These errors could cover up inter-observer variation. A high rank correlation (maximum $=1$ ) means that the recordings are ordered (almost) identically by both observers, and that there is no - or hardly any - other variation.

For both tests a p-value $<0.05$ was considered statistically significant.

The quality judgement of the AFP recordings and PDC plateaus made by each of two observers was related to the absolute difference between the AFP scores of both observers. If the absolute difference is large it might be expected that at least one of the two observers judged the AFP recording or the PDC's as 'moderate' or 'bad' (4 or 5 on the ordinal scale). In that case the correlation coefficient would be large $(\geqslant 0.7)$ or at least positive. A p-value $<0.05$ was considered statistically significant.

The balanced study design offered the possibility of performing a variance-components analysis. In groups I and II one recording for each of the 15 patients was available with three PDC's in each recording. Judged by four observers this is equal to $15 \times 3 \times 4=180$ observations. In group III at least one recording for each patient was available with three PDC's in each recording. Judged by four observers this results in $35 \times 3 \times 4=420$ observations.

Total variance (= squared standard deviation) is the sum of the following sources of variations: inter- and intra-patient variation, inter-observer variation and all other sources of interpretation errors including intra-observer variation, although not separately tested in this study.

\section{RESULTS}

The results of the various testing procedures will be represented for the group of normal children (groups I and II) and the group of patients (group III) separately.

\section{Normal newborns and infants (Groups I and II)}

Five recordings of a total of five newborns of group I and sixteen recordings of a total of ten infants of group II were evaluated by the four observers.

\section{Inter-observer variation}

as fixed effect

Difference in AFP scores between each 
TABLE I: Inter-observer variation analysis in both groups of normal children (groups I and II) ( $\mathrm{n}$ = number of evaluated AFP recordings).

\begin{tabular}{l|c|c|c|c|c|} 
Observer pair & $\mathbf{n}$ & $\begin{array}{c}\text { Mean AFP scores } \\
\pm \mathrm{SD}\end{array}$ & $\begin{array}{c}\text { Mean } \\
\text { difference } \\
(\mathbf{m m H g})\end{array}$ & $\begin{array}{c}\text { SE mean } \\
\text { difference } \\
(\mathbf{m m H})\end{array}$ & $\begin{array}{c}\mathbf{p} \\
\text { (Wilcoxon) }\end{array}$ \\
\hline 1 and 2 & 21 & $10.6 \pm 2.4-10.6 \pm 2.2$ & 0.0 & 0.2 & 0.59 \\
1 and 3 & 21 & $10.6 \pm 2.4-11.4 \pm 2.6$ & -0.8 & 0.3 & 0.01 \\
1 and 4 & 21 & $10.6 \pm 2.4-10.7 \pm 2.2$ & -0.1 & 0.2 & 0.49 \\
2 and 3 & 21 & $10.6 \pm 2.2-11.4 \pm 2.6$ & -0.8 & 0.3 & 0.01 \\
2 and 4 & 21 & $10.6 \pm 2.2-10.7 \pm 2.2$ & -0.1 & 0.1 & 0.34 \\
3 and 4 & 21 & $11.4 \pm 2.6-10.7 \pm 2.2$ & 0.7 & 0.3 & 0.01
\end{tabular}

two observers, mean difference, standard error of mean difference (SE) and p-value are shown in Table I.

This Table shows that observer 3 scored significantly higher than observers 1,2 and 4.

\section{Error variation test}

The Spearman rank correlation coefficient between any two observers was greater than 0.8. Hence no large error variation was found.

\section{Quality judgement}

\section{and absolute observer difference}

The relationship of the absolute difference between any two observers in the interpretation of the AFP recordings with the quality judgement of the recording and the PDC plateaus was also investigated.

Positive correlations were expected, however both negative and positive correlations were found. The negative correlation coefficients ranged from - 0.09 to -0.03 between both observer pair 1, 4 and observer pair 2, 3 and 2, 4. The positive correlation coefficients varied from 0.06 to 0.37 and none of them was statistically significant. It means there was no significant correlation between the absolute difference between any two observers on the one hand and the judged recording quality on the other.

\section{Variance components analysis}

From a variance components analysis with fifteen infants 'measured' by four observers it was estimated that $3 \%$ of the total variance of the AFP score could be attributed to observers, $87 \%$ to infants and the remaining $10 \%$ to errors. Here the AFP score was defined as the average of the AFP level calculated from three PDC's. This averaging could cover up intra-patient variation which could be distinguished if the AFP value, calculated from one single PDC, was used. With the variance components analysis the various components could be estimated, now having three PDC's per infant judged by four observers. The estimated variance was 18.9 (=100\%). The estimated components were $5.5(=29 \%)$ inter-patient variation, $11.5(=61 \%)$ intra-patient variation, 0 $(=0 \%)$ inter-observer variation and the remaining $10 \%$ representing measurement error.

\section{Patients (group III)}

Sixty-one recordings of a total of 35 patients were evaluated by the four observers. 
TABLE II: Inter-observer variation analysis in the group of patients (group III). ( $n=$ number of evaluated recordings).

\begin{tabular}{l|c|c|c|c|c|} 
Observer pair & $\mathbf{n}$ & $\begin{array}{c}\text { Mean AFP scores } \\
\pm \mathrm{SD}\end{array}$ & $\begin{array}{c}\text { Mean } \\
\text { difference } \\
(\mathbf{m m H g})\end{array}$ & $\begin{array}{c}\text { SE mean } \\
\text { difference } \\
(\mathbf{m m H g})\end{array}$ & $\begin{array}{c}\mathbf{p} \\
\text { (Wilcoxon) }\end{array}$ \\
\hline 1 and 2 & 61 & $16.4 \pm 6.0-14.9 \pm 5.4$ & 1.2 & 0.2 & $<0.01$ \\
1 and 3 & 61 & $16.4 \pm 6.0-16.7 \pm 6.1$ & -0.3 & 0.2 & 0.03 \\
1 and 4 & 61 & $16.4 \pm 6.0-16.1 \pm 6.1$ & -0.3 & 0.2 & 0.11 \\
2 and 3 & 61 & $14.9 \pm 5.4-16.7 \pm 6.1$ & -1.7 & 0.2 & $<0.01$ \\
2 and 4 & 61 & $14.9 \pm 5.4-16.1 \pm 6.0$ & -1.1 & 0.3 & $<0.01$ \\
3 and 4 & 61 & $16.7 \pm 6.1-16.1 \pm 6.0$ & 0.6 & 0.2 & 0.01
\end{tabular}

\section{Inter-observer variation} as fixed effect

Difference in AFP scores between pairs of observers, mean difference, standard error of mean difference (SE) and p-value are shown in Table II.

This Table shows that observer 1 did not score significantly higher than observer 4; however she scored significantly higher than observer 2 and significantly lower than observer 3. Furthermore observer 2 scored significantly lower than observer 3 and 4 and observer 3 scored significantly higher than observer 4 .

\section{Error variation test}

The Spearman rank correlation coefficient between all pairs of observers was greater than 0.95 . Hence hardly any error variation was found.

\section{Quality judgement}

and absolute observer difference

The relationship of the absolute difference between any two observers in the interpretation of the AFP recordings with the quality judgement of the recording and the PDC plateaus was also investigated. The correlation coefficient ranged from 0.01 to 0.27 and was negative once. It means that no significant correlation could be established between the absolute observer difference and the judged recording quality.

\section{Variance components analysis}

From a variance components analysis with 35 patients 'measured' by four observers it was estimated that $35 \%$ of the total variance of the AFP score could be attributed to observers, $63 \%$ to patients and the remaining $2 \%$ to errors. This averaging could cover up intra-patient variation which could be distinguished if the AFP value calculated from one single PDC was used. With the variance components analysis the several components could be estimated now having three PDC's per patient judged by four observers. The estimated variance was $69.9(=100 \%)$. The several estimated components were 30.7 $(=44 \%)$ inter-patient variation, 25.2 $(=36 \%)$ intra-patient variation, 7.4 $(=11 \%)$ inter-observer variation and the remaining $9 \%$ representing measurement error. 


\section{DISCUSSION}

As described in chapters 3,4 and 5 the technique for measuring AFP with the RTT is reliable and reproducible. In a clinical situation, decisions to shunt or not to shunt a child are based on clinical symptoms and brain imaging techniques. Monitoring of intracranial pressure on children with an open fontanelle provides additional information. It is important that the AFP recordings can be interpreted reliably with regard to the actual level of pressure. To test whether the recordings are interpreted differently by persons who are either familiar or not familiar with the technique, the inter-observer analysis was carried out.

Both in the group of normal infants (groups I and II) and in the group of patients (group III) the inter-observer variation was first tested as a fixed effect. In the group of normal infants the Wilcoxon test showed there was a systematic difference between one observer and the other three observers. In the group of patients the Wilcoxon test showed there was a systematic difference between several observers. In both groups the inter-observer differences were small and within the measurement error and therefore of no clinical relevance. Both in the group of normal children and in the group of patients only a small error variation (including intra-observer variation) was established.

Whether the absolute difference in AFP scores between any two observers was related to each observer's judgement about the quality of the recording and the PDC plateaus was statistically evaluated.

The rank correlations were predominantly positive as was expected: a large difference between any two observer's scores of a patient's AFP tends to comply with each observer's judgement of the AFP recording as being of bad quality. However, the rank correlations were small $(<0.27)$ and not statistically significant, therefore no significant relation between quality judgement and random inter-observer variation in AFP recording was established.

The balanced study design offered the possibility of looking more precisely into the contribution of each of the four variation sources. The four variation sources were: the inter-patient variation represented by the patient, the intra-patient variation represented by the PDC, the interobserver variation represented by the observer, and all other error sources defined as the measurement error. The PDC's were regarded as three separate measurements. These three PDC's of one patient were never interpreted independently since they were present consecutively in one recording. Therefore the PDC represents short term intra-patient (or biological) variation.

With a variance components analysis it was possible to estimate the total variance and its several contributing components as if one PDC had been judged by any one of the four observers. Both in the group of normal children and that of patients the largest percentage of variation sources is caused by the inter- and intra-patient variations. The inter-observer variation was very small in the group of normal infants and only $11 \%$ in the group of patients. The errors were small in both groups.

The study design was not suitable for testing the intra-observer variation separately, since the three PDC's could not be judged independently by one observer. However, it is tempting to make a few statements about this element.

Both the intra-patient and the inter-observer variation contain an element of 
intra-observer variation. To define this intra-observer variation more precisely the same observer should have assessed the same PDC or the same recording at least twice and independently, e.g. blind and with an interval between the assessments. This aspect was not examined so no direct statement can be made about intra-observer variation. Indirectly, it can be said that since the inter-observer variation is small it is to be expected that the intraobserver variation is also small. Furthermore the error variation was small in both the group of normal infants and the group of patients.

If one observer assessed three PDC's per patient then the greatest percentage of the total variance components is represented by the patient (inter- and intra-patient variation). This percentage was calculated as $87 \%$ in the group of normal infants and as $63 \%$ in the group of patients.

The observer component (inter- and intraobserver variation) was $3 \%$ in the group of normal infants and 35\% in the group of patients. Although the percentage is greater in the patient group the mean differences in actually scored AFP level are so small that this is not clinically relevant. In both groups (normal infants and patients) the measurement error was small, most likely due to standardized and carefully carried out interpretation of the AFP recordings.

\section{CONCLUSIONS}

1 The inter-observer variation as fixed effect in the interpretation of an AFP recording in the group of normal children showed a small systematic difference between one observer and the other three observers. In the group of patients this inter-observer variation showed a small systematic difference between several observers. The actual differences in AFP scores are of no clinical relevance.

2 The inter-observer variation as random effect in the interpretation of an AFP recording was estimated 'small' to 'acceptable' both in the group of normal infants and in the group of patients.
3 The error variation in the interpretation of the level of AFP from an AFP recording is small.

4 There was no significant relationship between the error variation and the observer's subjective judgement about the quality of the AFP recording and of the PDC plateaus.

5 The intra-observer variation, although not investigated separately, was indirectly estimated as small.

6 There is a substantial intra-patient (or biological) variation; in order to characterize the ICP of a patient, the average of at least three PDC's has to be taken as the AFP score of that patient. 


\section{H A P T E R 7}

\section{ANTERIOR FONTANELLE PRESSURE MONITORING IN GLINICAL PRACTICE}

\section{INTRODUCTION}

Monitoring of intracranial pressure on children is a normal part of extensive monitoring in pediatric intensive care wards in cases of head injury, metabolic and hypoxic- ischaemic encephalopathy, other causes of brain oedema and intracranial haemorrhages (Mickell and Ward 1984). The techniques used are often invasive, like ventricular catheters and subarachnoid or subdural catheters. Although the devices were usually adapted for pediatric use the procedures all have the main disadvantage of infection risk (see chapter 2).

Non-invasive measurement of ICP is a valuable tool in the care for critically ill neonates and infants (Raju et al. 1980, Bada 1983, McIntosh 1983, Newton 1987).

In daily clinical practice anterior fontanelle pressure monitoring is not routinely used. This is partly due to the fact that not all clinicians recognize the advantage of measuring ICP and that, with the present techniques, anterior fontanelle pressure monitoring is not easy to perform.

In certain clinical problems AFP monitor- ing could be a valuable contribution. A CSF shunt may be indicated in children with hydrocephalus and clinical signs of increased ICP and may be considered in children with macrocephaly or a too fast growing skull without clinical signs of increased ICP. The question 'to shunt or not to shunt' is not always easy to answer. Sometimes there may be justification for waiting some time before initiating operative procedures. AFP monitoring might contribute towards these clinical decisions.

The value of AFP monitoring with the RTT in clinical practice had to be established. The inventors of a new technique and a person who performs the clinical application are usually biased in making statements about the usefulness of the method in clinical practice. Therefore we asked and tested the opinion of a nonbiased clinician with regard to the question 'to shunt or not to shunt' in order to examine the role of AFP monitoring.

\section{MATERIAL AND METHODS}

A skilled child neurologist reviewed the clinical data of the 141 patients of this study (chapter 5), without knowing the name of the patient, the results of the AFP measurements and whether the child had been eventually shunted or not. She re- ceived written documentation about the patients consisting of the first anonymous discharge letters usually concerning a period of two to four months. Information about decisions to shunt or not to shunt were left out of the discharge letters. To 
TABLE I: Checklist for the evaluation of the clinical information.

\author{
Medical History \\ behavioral changes \\ feeding/eating problems \\ insufficient weight gain \\ vomiting \\ drowsiness
}

\section{Neurological Examination}

skull circumference above 98 th percentile

skull circumference increasing too fast

bulging or tense fontanelle

sunset eyes

convergent strabism

unequal size of pupils

papilloedema

muscle tone of the legs

knee and ankle jerks

Babinski sign (right or left)

\section{Results of brain imaging techniques}

CT-scan of the skull (*) internal hydrocephalus external hydrocephalus subdural effusions

Cranial ultrasound $(*)$

enlarged lateral ventricular index

$\begin{array}{ll}\text { yes } & \text { no } \\ \text { yes } & \text { no } \\ \text { yes } & \text { no } \\ \text { yes } & \text { no } \\ \text { yes } & \text { no }\end{array}$

unknown

unknown

unknown

unknown

unknown

$\begin{array}{lll}\text { yes } & \text { no } & \text { unknown } \\ \text { yes } & \text { no } & \text { unknown } \\ \text { yes } & \text { no } & \text { unknown } \\ \text { yes } & \text { no } & \text { unknown } \\ \text { yes } & \text { no } & \text { unknown } \\ \text { yes } & \text { no } & \text { unknown } \\ \text { yes } & \text { no } & \text { unknown } \\ \text { low } & \text { normal } & \text { high } \\ \text { low } & \text { normal } & \text { high } \\ \text { yes } & \text { no } & \text { unknown }\end{array}$

\section{*CT-scan}

The conclusion 'internal or external hydrocephalus' was based on the judgement of the neuroradiologist of the University Hospital of Rotterdam and on estimating the central and peripheral CSF spaces (Skjodt et al. 1988).

The CT-scan was interpreted as showing internal hydrocephalus, when the lateral ventricles were dilated, according to Evans ratio $>0.35$ (Yamada 1983).

The CT-scan was interpreted as showing external hydrocephalus, when the bifrontal subarachnoid spaces and the frontal interhemisferic fissure were widened, together with mild enlargement of the remainder of the subarachnoid space; mild to moderate ventriculomegaly could be present at the same time (Alvarez et al. 1986, Maytal et al. 1987).

The CT-scan was interpreted as showing 'subdural effusions', when manifest subdural fluid collections were present either in the frontal or occipital regions.

$* *$ Ultrasound

The lateral ventricular index is equal to the quotient of the width of both lateral ventricles at the level of the cella media and the diameter of the skull. Normal values in infants are 0.30 to 0.35 (Baerts 1984). 
enable an equal evaluation all information a checklist was made before-hand based on medical history, neurological examination and results of CT-scan and ultrasound of the skull. This checklist is presented in Table I. With this list she looked over all relevant information.

Having filled in the list she made one of the three following decisions:

shunt yes, no shunt, or no opinion.
The decision of the reviewing child neurologist was then compared with the clinical situation where a CSF shunt was given or not to that particular child in the same period. Whenever there was a difference in opinion the role of AFP monitoring was examined. Furthermore, the complete patient data and the checklist, filled in by the child neurologist, were reviewed to find factors explaining this difference.

\section{RESULTS}

The patients were categorized in five groups. Each group was based upon two items, 1 decision to shunt or not or no opinion (based on retrospectively reviewed clinical information), 2 shunting operation performed or not. In Table II these groups and the number of patients in each group are shown.

There was consensus in 129 of 141 cases (92\%): 46 infants were operated (group A) and 83 infants were not operated (group B). In 12 cases $(18 \%)$ the decisions were dissimilar. For five infants was decided not to perform an operation after studying the clinical data; actually they were shunted (group C). Four infants in whom a drain was considered necessary by the child neurologist were not operated (group D). In three cases the reviewer could not make a decision and so she chose for: no opinion. These three patients were actually shunted (group E).

\section{Description of groups and patients}

GROUP A (decision to shunt and operated).

This group consisted of 46 infants; 21 boys and 25 girls. In Table III the diagnoses of these children are represented.

A total of 94 AFP measurements were

TABLE II: Number of patients categorized in groups according to the decision of the reviewing child neurologist to shunt, and the actual clinical situation.

\begin{tabular}{|c|c|c|c|c|c|}
\hline & \multicolumn{5}{|c|}{ Decision to shunt based on the review of clinical information } \\
\hline & Shunt & Yes & No & No opinion & Total \\
\hline \multirow{3}{*}{$\begin{array}{l}\text { Clinical } \\
\text { situation }\end{array}$} & Yes & $46(\mathrm{~A})$ & $5(\mathrm{C})$ & $3(\mathrm{E})$ & 54 \\
\hline & No & $4(\mathrm{D})$ & 83 (B) & - & 87 \\
\hline & Total & 50 & 88 & 3 & 141 \\
\hline
\end{tabular}

() = group-identification, see text. 
TABLE III: Number of patients in group A categorized according to relevant clinical diagnosis.

myelodysplasia with (developing) hydrocephalus 11

aquaductal stenosis $\quad 2$

arachnoid cysts 2

cerebral arterio-venous malformation and hydrocephalus 1

chromosomal disorder and hydrocephalus 1

Gorlin's disease with hydrocephalus $\quad 1$

choroid plexus papilloma

hydrocephalus (tri-,quadriventricular) of unknown cause 9

macrocephaly and internal or external hydrocephalus on CT-scan 4

hydrocephalus after perinatal haemorrhage ( 7 of 9 infants were prematures) 9

hydrocephalus after bacterial meningitis 2

craniosynostosis with stenosis of more than two sutures 3

Total 46

performed. Eighteen children were measured once, 19 children were measured twice, three children were measured three times, four children were measured four times, one child was measured six times and one child was measured seven times. Of these measurements 27 continuous AFP measurements were performed in 15 children. All AFP measurements were performed from the age of 30 weeks gestational age to 11 months calendar age. In one exceptional case, a boy (patient 136 with Pfeiffer syndrome), AFP was measured at the age of eight years. He had multiple skull defects after several operations for correction of a craniosynostosis and for a developing hydrocephalus. Details and a figure representing part of the AFP registration were given in chapter 5 . All 46 children had clinical signs of increased ICP i.e. a fast growing skull circumference rate, a tense fontanelle, sunset phenomenon, frequent vomiting or drowsiness. The average pre-operative $\mathrm{AFP}$ level was $20.6 \pm \mathrm{SD} 7.6 \mathrm{mmHg}$.

GROUP B (decision not to shunt and not operated).
This group consisted of 83 children; 58 boys and 25 girls. In Table IV the diagnoses of these children are given.

In these 83 children 127 AFP measurements were performed. Fifty-eight children were measured once, 17 children were measured twice, four children three times, two children four times, one child six times and one child nine times. In 27 children 39 continuous AFP measurements were performed. The measurements were performed from 30 weeks gestational age to 24 months calendar age. Because of the heterogeneity of this group we did not calculate an average level of AFP. None of the 83 children had clinical signs of raised ICP when the child neurologist reviewed the clinical information.

However, during the follow up period (of which information still was not available at the time of the review) 12 infants developed clinical signs of raised ICP and they were shunted on clinical grounds. Five of these infants had a myelodysplasia (patients 77,125,140,141 and 144). The spinal defect was closed the day after birth. At discharge, 4-6 weeks after operation they had no clinical signs of raised ICP. 
TABLE IV: Number of patients in group B categorized according to relevant clinical diagnosis.

non-specific psychomotor retardation + leucodystrophy

$\begin{array}{ll}\text { macrocephaly without CT-scan abnormality } & 7\end{array}$

macrocephaly with internal hydrocephalus on CT-scan $\quad 2$

macrocephaly with external hydrocephalus on CT-scan $\quad 7$

macrocephaly with subdural effusions on CT-scan 1

hydrocephalus (tri-, quadriventricular) of unknown cause $\quad 2$

(congenital) cerebral malformations and abnormalities 6

(Dandy Walker, agenesis of corpus callosum, oculomotor apraxia, porencephalic cyst)

perinatal haemorrhages (subdural, subarachnoid, thalamic, 10

intracerebral and intraventricular bleeding)

craniosynostosis of one or two sutures $\quad 5$

myelodysplasia $\quad 7$

seizures 2

head trauma and skull fractures $\quad 1$

bacterial meningitis $\quad 4$

haemorrhagic shock encephalopathy 1

Klippel Trenaunay syndrome $\quad 1$

Sturge Weber syndrome $\quad 1$

near sudden infant death syndrome $\quad 1$

(congenital) cardiac abnormalities $\quad 8$

(congenital) gastro-enterological diseases $\quad 5$

miscellaneous congenital syndromes

neuroblastoma 1

premature birth 1

The ultrasound of the skull showed enlarged ventricles. On average by the age of three months they developed clinical signs of increased ICP such as a tense fontanelle and an increasing skull circumference. AFP measurements performed at that time showed in all cases pressures above $20 \mathrm{mmHg}$ (with an average pulsation amplitude of $5 \mathrm{mmHg}$ ), confirming the clinical diagnosis of raised ICP.

Three patients (21, 43 and 104) were diagnosed Klippel Trenauny syndrome, IVH grade IV at a premature birth of 31 weeks and Apert's syndrome respectively. At the time of reviewing the clinical information there were no obvious signs of raised ICP. During the follow up period, by the age of six months, they developed clinical signs of raised ICP such as too rapidly growing skull circumference, a tense fontanelle, occasional vomiting and drowsiness. AFP measurements performed at that time gave pressures above 20 $\mathrm{mmHg}$ with an average pulsation amplitude of $8 \mathrm{mmHg}$, this being in accordance with the clinical diagnosis of raised ICP. One child (patient 99) had an external hydrocephalus on the CT-scan. At the time of reviewing the clinical information there were mild signs of increased ICP like a large, tense fontanelle and macrocephaly. During the follow up period the child was 
again admitted to hospital at the age of five months with severe bilateral frontal subdural haemorrhages, skull fractures, anaemia and shock, probably due to battering. He was comatose and had other clinical signs of raised ICP like a very tense fontanelle, macrocephaly and a vertical gaze paralysis. AFP monitoring revealed pressures of $39.3 \mathrm{mmHg}$ with pulsation amplitudes of 8-13 mmHg. He underwent several fontanelle punctures to vent the subdural blood. Afterwards he was operated for a subduro-peritoneal shunt, after which the clinical signs of raised ICP disappeared. A postoperative AFP measurement showed a mean pressure of 10 $\mathrm{mmHg}$ in the continuous recording.

Three patients will be described more extensively because of the role of AFP monitoring.

Patient 16, male, term born after an uncomplicated pregnancy and delivery. $\mathrm{He}$ was admitted to hospital at the age of nine months to evaluate the cause of a severe psychomotor retardation, a spastic diplegia and visual disturbances. A CT-scan of the brain revealed a large porencephalic cyst in the left hemisphere. AFP measurement at that time showed a pressure of $25.1 \mathrm{mmHg}$ with pulsation amplitude of 4-7 mmHg. On clinical grounds (no tense fontanelle and no macrocephaly) the child was not shunted. Two months later the child was referred again because of an increasing skull circumference rate (from the 75 th percentile to above the 98th percentile) and an increased tension of the fontanelle. A new CT-scan was done. This revealed an increased volume of the porencephalic cyst. Before AFP measurement could be performed a ventriculo-cardiac shunt was implanted. During the follow up period of five years the child remained retarded and spastic unable to walk and talk. The skull circumference growth rate stabilized.

In this case the AFP measurement was already indicative of the raised ICP.

Patient 95, male, term born after an uncomplicated pregnancy. The delivery was complicated due to the narrow pelvic anatomy of the mother. The child had an Erb's paralysis and a fractured humerus of the right arm. He had an intracranial haemorrhage and a left parietal brain infarction. After a few weeks a CT-scan showed a developing internal hydrocephalus. An AFP measurement at the age of three weeks gave a (raised) pressure of 14.3 $\mathrm{mmHg}$ with a pulsation amplitude of 2 $\mathrm{mmHg}$. Fontanelle puncture was performed, the CSF pressure was $13 \mathrm{~cm} \mathrm{H} \mathrm{H}_{2} \mathrm{O}$ $=10 \mathrm{mmHg}$. After removal of $10 \mathrm{cc}$ CSF the pressure was $2 \mathrm{~cm} \mathrm{H}_{2} \mathrm{O}=1.5 \mathrm{mmHg}$. The AFP measurement performed after 2 hours showed a pressure of $7.6 \mathrm{mmHg}$. The child was discharged in good condition without signs of increased intracranial pressure. At the age of five months he developed signs of raised intracranial pressure with sunset sign and a tense fontanelle. An AFP measurement revealed a pressure of $29.5 \mathrm{mmHg}$ with pulsation amplitudes of 5-7 $\mathrm{mmHg}$. A shunt was implanted. AFP measurement performed one week after the operation gave pressures of $12.5 \mathrm{mmHg}$ with pulsation amplitudes of 3-5 mmHg. In the follow up period of one year his psychomotor development was good.

In this case the first AFP measurement already indicated a slightly raised ICP.

Patient 108, male, was born after a gestational age of 32 weeks, as one of twins. During pregnancy and delivery there were no complications. At the age of six months he was admitted to hospital to evaluate the cause of his psychomotor retardation and macrocephaly. The CT-scan of the 
skull showed an external hydrocephalus. AFP monitoring gave pressures of 23.3 $\mathrm{mmHg}$ with pulsation amplitudes of 6-7 $\mathrm{mmHg}$. A continuous monitoring of 5 hours was performed of which only 18 minutes could be interpreted. The average level of AFP was $17.0 \mathrm{mmHg}$. During this time the child slept. $100 \%$ B-waves were recorded with amplitudes of $2-5 \mathrm{mmHg}$. A fontanelle puncture was performed and $5 \mathrm{cc}$ of fluid with a high protein level was vented. The pressure at that moment was $13 \mathrm{~cm} \mathrm{H}_{2} \mathrm{O}=9.6 \mathrm{mmHg}$. AFP after puncture was $16.1 \mathrm{mmHg}$ with pulsation amplitudes of 5-7 mmHg. He was discharged, however because of slow progressive skull circumference growth rate he was again admitted to hospital for observation. AFP monitoring revealed pressures of 17.2 $\mathrm{mmHg}$ during crying with pulsation amplitudes of 2-3 mmHg. A continuous monitoring of 10 hours could be performed while the child was quiet of which 6 hours could be interpreted. No B-waves were seen. The mean pressure was $10.3 \mathrm{mmHg}$. Based on clinical grounds (psychomotor retardation, progression of skull circumference) and the CT-scan results he was operated for a subduro-peritoneal shunt since the frontal CSF spaces were very large. This was interpreted initially as subdural effusions, later on however, as an external hydrocephalus. The child did quite well during the follow up period of one year. The skull circumference was growing parallelly, slightly above the 98 th percentile.

In this case AFP monitoring showed an elevated pressure in the initial recording. However, during follow up the pressure was lower and in the normal range. The question is whether this child really had to be operated. This will be discussed later on.
GROUP C (decision not to shunt and operated).

This group consisted of five infants; three boys and two girls. Fifteen AFP measurements were performed in total. In one infant one AFP measurement was performed, in two infants two measurements, in one infant three measurements and in one infant seven measurements were done. In two infants four continuous AFP measurements were performed. The AFP measurements were performed from the age of six days to nine months calendar age. Four of these patients had mild clinical signs of raised ICP and on clinical grounds they were shunted. The reviewing clinician probably misinterpreted the written clinical information. This information was scarce in all cases. However, signs of raised ICP were mentioned in two of them.

One patient will be described more extensively.

Patient 18, male, was born term after an uncomplicated pregnancy and delivery. At the age of four weeks he acquired a left sided skull fracture and a brain contusion after falling off a table. He made a good recovery and was discharged after three weeks. In follow up visits the skull circumference growth rate came slowly above the 98th percentile at the age of eight months. At neurological examination he had signs of retarded motor-development and a left sided spastic hemiplegia. The CT-scan of the skull showed bilateral subdural hygroma's in the occipital regions. AFP measurements at the age of nine months gave (normal) pressures of 11.8 $\mathrm{mmHg}$ with pulsation amplitudes of 3-4 $\mathrm{mmHg}$. Based on clinical grounds (psychomotor retardation and macrocephaly) and the results of the CT-scan it was decided to implant a bilateral subduroperitoneal drain. Postoperative AFP measurements gave pressures of $11 \mathrm{mmHg}$ 
with pulsation amplitudes of $3-5 \mathrm{mmHg}$. The child walked at the age of $2 \frac{1}{2}$ years. The control CT-scan at the age of $3 \frac{1}{2}$ years no longer showed subdural hygromas. The skull circumference at that time was at the 75 th percentile. The follow up period has been four years.

In this case the AFP measurements did not show a raised pressures. Whether the operation was necessary or not will be discussed later on.

GROUP D (decision to shunt and not operated).

This group consisted of four infants: three boys and one girl. Seven AFP measurements were done in total. In three infants one AFP measurement was done, and in one infant four AFP measurements were done. In one infant four continuous AFP measurements were performed. The age at which the fontanelle pressure measurements were performed ranged from two months to eleven months. These patients will be described.

Patient 11, male, was born preterm after a gestational age of 34 weeks. The pregnancy was uncomplicated. After birth he had a transient irregularity of breathing and a slight cardiac arrhythmia. He came to the outpatient clinic at the age of nine months with symptoms of too quickly increasing skull circumference rate and a slight psychomotor retardation. The neurological examination showed, besides a global retardation, no focal abnormalities. The CT-scan of the brain showed an external hydrocephalus. AFP monitoring revealed a pressure (in the normal range) of $12.9 \mathrm{mmHg}$. After neurosurgical consultation the decision was made not to shunt. In frequent follow up examinations there was improvement of the psychomotor skills. The skull growth rate decreased and tended to approach the 98 th percentile (see figure 1). At the age of 20 months

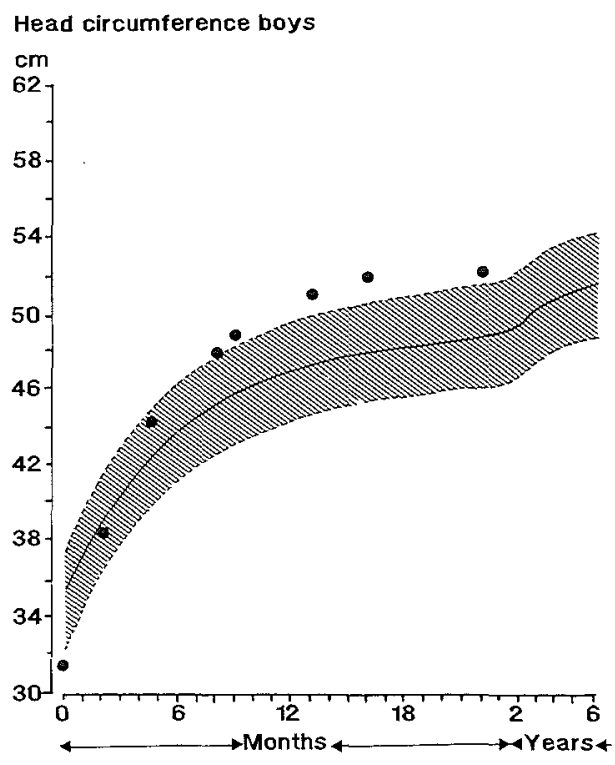

Fig. 1 Head circumference growth curve of patient 11 .

he walked without support and spoke short sentences.

In this case AFP monitoring revealed pressures in the normal range (mean AFP in group II, chapter $4,11.0 \pm 1.9 \mathrm{mmHg}$ ).

Patient 19, female, was born after a gestational age of 37 weeks. There were no complications during pregnancy and labour. After birth she was diagnosed as having Crouzon's syndrome. The CT-scan of the skull showed an internal hydrocephalus. At AFP measurement the pressure was $17.8 \mathrm{mmHg}$. She underwent a first craniofacial operation at the age of seven months. At the age of 15 months a second corrective operation was done. A second CT-scan showed no progression of the slight internal hydrocephalus. After four years follow up her psychomotor development was normal. Her head circumference was on the 98th percentile.

In this case AFP measurement showed an elevated pressure before the craniofacial operation. After operation, during the 
follow up period she showed no clinical signs of increased pressure.

Patient 40, male, was born term with a large omphalocele and hypoplasia of lungs and chest after an uncomplicated pregnancy and delivery. The congenital anomalies were treated operatively. Thereafter many complications occurred. During a period of congestive heart failure the skull circumference increased too fast from the 25 th to the 50th percentile. The CT-scan of the brain showed an external hydrocephalus. AFP measurement revealed a pressure of $29.2 \mathrm{mmHg}$ with pulsation amplitudes of 3-4 mmHg. It was decided not to shunt and to wait until the internal complications were treated. On follow up, the skull circumference growth rate did not increase. His psychomotor development was normal after four years. In these years there were many operations to correct the congenital anomalies.

In this case the AFP measurement revealed a (probably transient) high pressure level during a period of congestive heart failure. This will be discussed later on.

Patient 67, male, was born after a gestational age of 35 weeks with a major diaphragmatic hernia. Pregnancy was uncomplicated but delivery was done by vacuum extraction. He was intubated and operated. After this operation a cardiac arrest developed with a duration of approximately five minutes, which was appropriately treated. One week later generalized seizures were seen which were treated successfully with anti-epileptic drugs. Pulmonary and liver problems evolved. Eventually, he was extubated after five months. The psychomotor development of this child was slow. Furthermore, the skull circumference started to grow progressively from the 50th to the 98th percentile. The CT-scan showed an external hydrocephalus. An AFP measurement at the age of $5 \frac{1}{2}$ months showed a pressure of $13.4 \mathrm{mmHg}$ with pulsation amplitudes of $4-5 \mathrm{mmHg}$. A continuous AFP monitoring was done over ten hours. Because of movement artifacts only $5 \frac{1}{2}$ hours of this registration could be interpreted. The average pressure was $12.9 \mathrm{mmHg}$. Thirty two percent of B-waves occurred, mostly during sleep, with a mean amplitude of $3 \mathrm{mmHg}$. Two A-waves could be seen with base pressures and maximal pressures of 12.5 and 12.1, and 26 and $33 \mathrm{mmHg}$ respectively. These waves had durations of 9 and of 12 minutes. A shunting operation was omitted because of the child's pulmonary problems. $\mathrm{He}$ was discharged at the age of six months.

During an admission for an oxygen saturation measurement an AFP measurement was done and showed a pressure of 10.5 $\mathrm{mmHg}$. A continuous registration of $10 \frac{1}{2}$ hours was performed. Because of many movement artifacts only one hour could be interpreted. The average pressure was $10 \mathrm{mmHg}$. Nine percent B-waves occurred with a mean amplitude of $1.8 \mathrm{mmHg}$. During the follow up period the psychomotor development improved and the skull circumference did not increase further. At the age of nine months a third AFP measurement was done during an acute admission to hospital for bronchitis and pulmonary hypertension. The AFP was $24.7 \mathrm{mmHg}$. A continuous registration for 10 hours took place, of which 3 hours could be interpreted. The average pressure was $16.7 \mathrm{mmHg}$. Sixteen percent B-waves with a mean amplitude of 4.5 $\mathrm{mmHg}$ were seen. After improvement of the clinical condition he was discharged. His psychomotor development improved slowly. The skull circumference growth rate decreased and started to grow along the 75 th percentile. At the calendar age of eleven months a new AFP measurement 
was done, showing a mean pressure of 13.7 $\mathrm{mmHg}$. A continuous measurement of 4 hours was done of which $3 \frac{1}{2}$ hours were interpretable. The average pressure was $12.7 \mathrm{mmHg}$, no B-waves were observed. The child's psychomotor development improved further, he walked at the age of 20 months.

In this case the AFP measurements gave normal pressure values and elevated pressures during a period of pulmonary hypertension. This will be discussed later on.

GROUP E (no opinion and operated). This group consisted of three boys. Ten AFP measurements in total were done; one boy was measured twice, one boy three times and one boy five times. Of these measurements 5 continuous AFP measurements were performed in these three boys. The age at which AFP measurements were performed ranged from 34 weeks gestational age to 11 months calendar age.

The clinical data available for retrospective judgement were considered insufficient to make a sound decision.

Two of these patients will be described. Patient 135, a term born male, with Crouzon's syndrome. During pregnancy and delivery there were no specific complications. Due to an anatomic deformation in the maxillary region the child had continuous respiratory problems. Furthermore, clinical signs of increased intracranial pressure were present. A CT-scan of the brain showed no severe hydrocephalus. However, during the short term AFP measurement the pressure was $19.2 \mathrm{mmHg}$ with pulsation amplitudes of $5-6 \mathrm{mmHg}$. A continuous AFP monitoring of $2 \frac{1}{2}$ hours was done resulting in an interpretable part of one hour. The average pressure was $27.5 \mathrm{mmHg}$. B-waves were seen in $42 \%$ of the total time with a mean amplitude of $6.9 \mathrm{mmHg}$. At the age of
$2 \frac{1}{2}$ months a craniofacial corrective operation was performed in the frontal region. Cranial ultrasound after operation revealed a progressive internal hydrocephalus. AFP measurement gave a pressure of 19.3 $\mathrm{mmHg}$ with pulsation amplitudes of 8-10 $\mathrm{mmHg}$. A second cranial corrective operation was scheduled to release the occipital bones. Postoperative cranial ultrasound still showed an internal hydrocephalus after which the decision was made for a shunting operation. A control AFP measurement one day after the operation still gave a high pressure of $20.5 \mathrm{mmHg}$. However, the pulsation amplitudes had decreased to 3-4 $\mathrm{mmHg}$. In a continuous monitoring of 3 hours and 15 minutes with an interpretable part of $1 \frac{1}{2}$ hour, the average pressure was $17.5 \mathrm{mmHg}$. B-waves occurred in $23 \%$ with a mean amplitude of $5.0 \mathrm{mmHg}$. Afterwards the fontanelle became less tense and the child was doing rather well. Further AFP measurements were not performed.

In this case the AFP measurement was indicative for raised ICP, even after two craniofacial operations. The progressive hydrocephalus was treated, and postoperatively the pulsation amplitude had decreased.

Patient 139 , male, born after a gestational age of 31 weeks. During pregnancy the membranes ruptured, during delivery there were no specific problems. The child developed an intraventricular bleeding with parenchymal extension (grade IV) a few days after birth. Follow up cranial ultrasound showed a slowly progressive hydrocephalus, at first treated with repeated lumbar punctures. The skull circumference growth rate increased from the 75th to the 98th percentile. During this period several AFP measurements were done. At 34 weeks gestational age the pressure was $3.1 \mathrm{mmHg}$ with a pulsation 
amplitude of $0.5 \mathrm{mmHg}$. At 35 weeks the pressure was $4.8 \mathrm{mmHg}$ with a pulsation amplitude of $0.5 \mathrm{mmHg}$. This measurement was continued for 45 minutes of which 30 minutes could be interpreted. The average pressure was $6.7 \mathrm{mmHg}$. No $\mathrm{B}$-waves were observed. The clinical condition was excellent and the child was discharged at the gestational age of 38 weeks. Follow up AFP measurement was done at the age of 40 weeks (term). The pressure was $8.8 \mathrm{mmHg}$ with pulsation amplitude of 1.5-2 mmHg. At the calender ages of four and five weeks AFP measurements were done revealing pressures respectively of 15.0 and $14.6 \mathrm{mmHg}$ and pulsation amplitudes of 3-4 and $4 \mathrm{mmHg}$. The skull circumference now came above the 98 th percentile and cranial ultrasound showed progressive hydrocephalus, and it was decided to give the child a vertriculoperitoneal shunt after which he did well. Figure 2 shows the skull circumference curve of this infant.

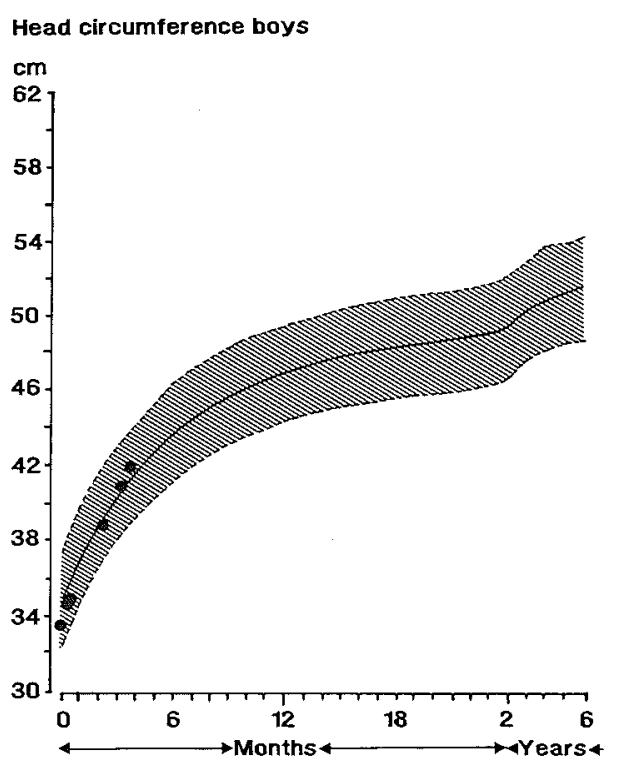

Fig. 2 Head circumference growth curve of patient 139.

In this case the usefulness of follow up AFP measurements is obvious in order to detect an early rise in ICP.

\section{DISCUSSION}

After having completed this part of the study it was interesting to see that in $92 \%$ of cases (129 out of 141) consensus existed between the reviewing child neurologist and the actual clinical situation in which a shunting operation was performed or not. The role of AFP monitoring in the various groups of patients and also in the individual patients mentioned above, will be examined to find out whether the results of the AFP measurements would have influenced the opinion of the reviewing child neurologist and also whether the clinical situation would have been different if the results of the AFP monitoring had been an essential tool together with the other examinations performed.
In group A (decision to shunt and operated), patients had clear clinical signs of raised ICP and the decision to perform a shunting operation needs no further discussion, the more so when the diagnoses in this group are reviewed (Table III). The results of the AFP measurements, performed in these patients, confirmed the clinical diagnosis.

Group B (decision not to shunt and not operated) can be compared to group A. However, of this group 12 patients were shunted during the follow up period. Nine patients developed clinical signs of raised ICP and, just as in group A, AFP monitoring merely confirmed the clinical diag- 
nosis. As already demonstrated in chapter 5, AFP measurements after a shunting operation showed lower pressures than the pre-operative levels. The three infants (patients 16,95 and 108) mentioned separately, need extra attention here because of the role of AFP monitoring. In patient 16 the AFP monitoring showed an elevated pressure level although the clinical signs were not considered severe enough to perform a shunting operation. In this case AFP monitoring could have played a valuable role in the decision to shunt, saving the time spent waiting for the clinical signs of raised ICP to develop.

In the case of patient 95 another useful aspect of AFP monitoring is shown. The first AFP measurement revealed a slightly elevated pressure. Based on one short term AFP measurement it is difficult to judge whether the raised ICP level is the cause of a permanent or a transient CSF circulation disturbance. It is very easy to perform AFP measurements in the outpatient ward and this child would have benefited from AFP measurements at regular intervals in order to detect the rise in ICP before the severe clinical signs of sunset phenomenon and a bulging fontanelle appeared.

In patient 108 a rather common clinical dilemma exists. The macrocephaly, the CT-scan results and the high protein CSF level supported the diagnosis of subdural fluid effusions. The short term AFP measurement showed elevated pressure levels, but in the second continuous AFP monitoring a normal mean pressure was recorded. The child was shunted. Reviewing the case history and the CT-scan it is doubtful whether this child had to be operated. Probably this child had so-called benign subdural fluid effusions (Naidich et al. 1976, Rothenberger and Brandl 1980a,b) which is one of the definitions of external hydrocephalus or benign communicating hydrocephalus (Kendall and Holland 1981). This syndrome is known to have a benign self limiting course. The continuous AFP monitoring could have played a valuable role in preventing the shunting operation. The initial high AFP level was probably due to a transient CSF circulation disturbance.

Usually a lumbar CSF infusion test is used to investigate the CSF dynamics such as CSF production, CSF absorption, compliance (dV/dP), outflow resistance (Ro) and pressure volume index (PVI), the latter being the volume necessary to increase CSF pressure by a factor 10 (Shulman and Marmarou 1971). To examine these parameters invasive techniques are necessary and in children these are not easily performed. Non-invasive AFP monitoring cannot provide for the same parameters. The technique is very suitable for follow up measurements in order to detect early rises in ICP even before the skull circumference rate increases or the ventricles enlarge. Furthermore, continuous monitoring is useful in detecting pathologic wave patterns resulting from cerebral vaso-instability. As the technique is simple to perform and harmless it is more easily applied than invasive, complicated infusion tests.

In group C (decision not to shunt and operated) four infants had clinical signs of raised ICP, in all cases confirmed by AFP monitoring. Due to the scarce written clinical information the reviewing child neurologist must have misinterpreted the clinical signs of raised ICP mentioned in the discharge letters.

Patient 18 needs extra attention. It is questionable whether the drain was really necessary. Pre- and post-operative AFP values did not differ. Whether the hygroma's would have disappeared without drainage remains an open question. 
The case-histories of the infants of group $D$ (decision to shunt and not operated) show that the results of the AFP monitoring could have been important in the decision of the reviewing child neurologist. Although there were mild clinical signs of raised ICP in patients 11 and 67, the AFP was in the normal range. In patient 19 there were clinical signs of raised ICP confirmed by AFP monitoring, probably due to a transient CSF circulation disturbance which normalized after the second craniofacial operation. In patients 40 and 67 there was a transient rise in ICP due to congestive heart failure and pulmonary hypertension respectively. The decision of the reviewing child neurologist at that moment is understandable, since she had no information on the follow up period due to the set up of the study. In this group the role of AFP monitoring would have been to prevent at least three shunt operations. Furthermore, in patients with cardiac and pulmonary disease the ICP is increased and the harmless follow up measurements together with CT-scans and ultrasound can help to determine whether this rise in ICP is transient or permanent.

In the three infants of group $\mathrm{E}$ (no opinion and operated) the judging clinician did not have enough relevant clinical information to make a sound judgement whether to shunt or not. In two of these children AFP monitoring proved to be a useful tool in follow up procedures to detect a rise in ICP or a permanent raised ICP. In one child AFP monitoring confirmed raised ICP and checked the drain function.

\section{CONCLUSIONS}

1 Regarding the various groups of patients and the role of the AFP measurements in the patients mentioned above, it can be concluded that AFP monitoring can be a useful tool in clinical practice. AFP monitoring confirms the clinical diagnosis of raised ICP, it can check drain function and during continuous monitoring pathologic wave patterns can be detected. In the Intensive Care Unit ICP can be monitored during longer periods continuously or intermittently. Furthermore, AFP monitoring can also be performed in the out-patient ward. During a follow up period AFP measurements can be performed at regular intervals. The results can help in determining whether or not shunting is necessary and if so when the shunting operation is best performed. This is useful in premature children with posthaemorrhagic hydrocephalus, in infants with craniosynostosis or myelodysplasia, in which a CSF circulation distur- bance can develop slowly and initially without clinical signs. However, AFP monitoring can detect early rises in ICP. AFP monitoring should not be used as the only valuable clinical parameter. It should be regarded as a harmless method to be used additionally to the neurological examination and brain imaging techniques.

2 In this retrospective study there was consensus in $92 \%$ between the decision 'to shunt or not to shunt' made by the child neurologist reviewing the clinical patient data and the actual clinical situation where shunting operations were performed or not. Only in $8 \%$ of cases was the decision of the reviewing child neurologist dissimilar from the actual clinical situation. This dissimilarity can be explained by information scarcity in the discharge letters and misinterpretation of clinical signs of raised ICP mentioned in these letters. Furthermore, there were difficulties in 
judging whether clinical signs of raised ICP were transient or permanent.

3 If mild clinical signs of raised ICP exist and the AFP level is within the normal range one should consider follow up AFP measurements first before considering shunting operation, in order to prevent unnecessary operations. The same holds for transient rises in ICP in cardiac or pulmonary diseases. 


\section{SUMMARY}

Since Quincke introduced in 1891 the lumbar puncture for measuring the pressure of the cerebral spinal fluid (CSFP) the interest in intracranial pressure (ICP) has remained constant throughout this century. With the introduction of continuous ICP recording by means of a ventricular catheter by Lundberg in 1960, it became possible to detect specific ICP wave patterns occurring under normal and pathological conditions. Also it became possible to perform direct dynamic studies of the craniospinal system. Löfgren (1973) and Marmarou (1975) studied thoroughly the relationship between volume and pressure of the craniospinal system, this resulting in the graphically established so called volume-pressure curve. Knowing the level of pressure only is not sufficient in order to understand brain function, since low or normal ICP levels $(<15 \mathrm{mmHg})$ can exist together with clinical signs of brainstem distortion due to large space-occupying lesions and high ICP levels $>20 \mathrm{mmHg}$ ) can exist without signs of neurological impairment.

In both adults and children there are several clinical indications that ICP should be measured, for instance in patients with head injury, or in the post operative management of neurosurgical patients, for the early detection of a rise in ICP due to cerebral oedema or a haematoma. In pediatric patients there are special indications that ICP should be measured such as intraventricular haemorrhage in prematures and newborns, (post-haemorrhagic) hydrocephalus, hypoxic-ischaemic encephalopathy, bacterial meningitis and Reye's syndrome.

Most techniques for measuring ICP are invasive and therefore clinicians are usually reluctant to use them on younger children, mainly because of the risk of infection and also because of the technical problems encountered when devices developed for adult patients have to be used on children. This is especially the case when longer monitoring periods are required. ICP monitoring will normally be done in intensive care units as part of the total patient monitoring.

The intracranial pressure can be invasively measured in several ways: CSF-pressure by lumbar, cisternal or ventricular puncture; pressure at the surface of the brain by subdural or subarachnoid catheters or a subarachnoid screw; intraparenchymal pressure by a pressure sensor; epidural pressure by means of several types of pressure sensors. Every type of measuring technique has specific advantages and disadvantages, and these are discussed in chapter 2. As the main disadvantage of all invasive techniques is the infection risk, non-invasive techniques were developed for measuring ICP in young children. In newborns and infants the anterior fontanelle affords a natural access to the intracranial compartment. The oldest known method for 'measuring' pressure is the palpation of the anterior fontanelle. This subjective method however is not considered a reliable way to quantify ICP.

For the quantification of the anterior fontanelle pressure (AFP) several techniques were developed during this century. A weight controlled tonometer for AFP monitoring was introduced in 1922 (Wentzler). Thereafter, an ocular tonometer was modified as fontanometer in 1959. (Davidoff and Chamlin). These devices were not very practical 
in daily use and also not very reliable for the exact quantification of AFP. Air-, or fluid-filled bags were used from 1964 onwards (Purin 1964, Brett 1966, Blaauw 1974), however these turned out not to be very reliable due to technical problems. AFP measuring devices based on the flow-switch principle (Numoto 1966) were described from 1978 onwards (Vidyasagar and Raju). Of all these types of devices one device became rather popular, the Ladd fiber optic device (Hill and Volpe 1981) in which the alteration in light absorption reflects the intracranial pressure. Solid state transducers acting according to the Wheatstone bridge principle were used from 1974 onwards (Wealthall and Smallwood 1974, Huch 1979). The latter two types of devices were considered more reliable for measuring pressure.

In all reports the fixation methods remained a major problem since one realized that fixation procedures could interfere with the actual measurement of pressure. To this aim a number of fixation methods were used, ranging from plaster of Paris, foam pads and ECG adhesive pads to special holding devices, all resting on the fontanelle itself.

In the beginning the methods for measuring AFP were based on the pulsations of the fontanelle (air filled bags and the first tonometer). Since these methods were not sufficient to quantify AFP exactly, more emphasis was laid upon the physical aspects of the fontanelle. The fontanelle, consisting of the skin plus underlying tissues was compared to a membrane covering a cavity (the intracranial compartment). Therefore the physical principle of measuring intracavitary pressure through an intact membrane seemed applicable for AFP monitoring. This principle had already been applied in obstetrics (Smyth 1957), and ophthalmology (Mackay 1964). For measuring pressure through an intact and flexible membrane it is necessary that the bending forces of the membrane are eliminated or at least reduced. This can be accomplished by aplanation or co-planarity, through which the membrane is flattened over a certain area, thereby reducing the membrane tension forces to practically zero. The technique for co-planar application of a transducer for ocular pressure measurements was described by Mackay (1964), who reported the appearance of a characteristic curve during co-planar application. This co-planarity technique was used in 1974 by Schettini who performed epidural pressure measurements through an intact dura. Schettini performed dynamic pressure tests by inserting and withdrawing a transducer at a constant rate. The graphically represented result of this test was the so called pressure depth curve (PDC). This PDC is essential for the AFP measuring technique used in this thesis.

In the University Hospital of Rotterdam an implantable telemetric device was developed for performing epidural pressure (EDP) measurements in patients with closed sutures (De Jong 1975, 1979). The principles of this transducer are described in chapter 3.

In analyzing the problems encountered by others who performed AFP monitoring and in observing the aspects of the fontanelle we noticed the similarity between epidural and fontanelle pressure measurements. The main difference lies in the skin (including underlying subcutaneous tissues) interposed between the dura and the transducer. The major problem in AFP measurements is the application pressure. The concept of controlled application pressure was exchanged for controlled depth setting. To this aim a special light weight perspex skull adaptor was designed, with facilities for accurate depth setting (chapter 3). The transducer was fitted in a perspex piston which 
can be moved up and down in the adaptor which in its turn is securely mounted on the skin overlying the bone adjacent to the fontanelle. A soft silicone fixation frame around the chin and occiput of the child and connected to a loading spring is used to maintain the adaptor accurately on the child's head. In this way any surplus of application force runs of to the skull. If necessary adhesive tape is used to prevent lateral dislocation. The measuring probe is loosely connected to the adaptor by means of a flexible wire which does not interfere with the movements of the child. With this construction the child can move around freely while AFP is being accurately measured.

As in epidural pressure measurements, accurate depth positioning of the transducer in AFP monitoring is equally important. Proper depth setting of the transducer is performed manually. An incorporated calibrated mechanical dial indicates insertion depth which is recorded on the same chart as the PDC's to exclude manual artifacts.

The procedure for AFP monitoring consists of the following steps: first the adaptor, containing the RTT, is secured to the skin surrounding the fontanelle by means of the silicone tubing of which the open ends are connected to the lever of the adaptor. There-after the calibration procedure is performed. Manual rotation of the piston in its thread leads to depth setting of the transducer. Just prior to the point at which the transducer touches the skin and no pulsations are seen, zero set points both for pressure and insertion depth are established on the chart, using the special bedside monitor dials (see chapter 3). By uniformly rotating the piston the insertion depth of the transducer is gradually increased and a characteristic PDC appears on the chart. Initially the pressure rises whereafter a plateau is reached at which cardiac pulsations remain constant. Here the transducer traverses the subarachnoid space and does not encounter extra opposing forces. Therefore a further rise of pressure is delayed. The plateau pressure thus recorded is equal to ICP. On further increasing the depth of the transducer a sharp rise in pressure is observed, due to compression of the subarachnoid space and contact with brain tissue. On subsequent withdrawal of the transducer the PDC is recorded in reverse, reaching the plateau pressure and eventually zero, as the transducer is again just beyond contact with the skin. When the RTT is repeatedly applied in this way, identical pressure recordings can be obtained each time.

With the use of the fixation frame both short term and long term (continuous) AFP measurements can be performed. For the continuous monitoring of AFP the transducer is set at the depth of the plateau of the PDC.

The level of ICP can be determined from the plateaus of the PDC's. Since there is a biological variation in the pressure, the AFP level was calculated as the average AFP level of the first three PDC's, where the AFP level from one PDC was calculated as the average of the ascending plateau and the descending plateau.

AFP monitoring with the RTT was tested in normal newborns and infants to establish normal values of AFP (chapter 4). In a group of 30 normal newborn babies aged 0-9 days, divided into age classes of two days, the mean AFP level ( \pm SD) was 8.9 $\pm 3.0 \mathrm{mmHg}$. No statistical difference between the several age classes could be established. In a group of 52 normal infants aged 30-330 days, divided into classes of 60 days, the mean AFP level was $11.0 \pm 1.9 \mathrm{mmHg}$. No statistical difference between 
the several age classes could be established. These normal values are within the range of normal values published by others using non-invasive techniques. The mean level of AFP in the group of 30 newborns differed statistically significant from the mean AFP level in the group of 52 infants. This age difference has also been reported by others both with invasive and non-invasive ICP measuring techniques. The explanation for the lower pressure levels in very young children compared to the pressure levels in slightly older children remains unclear. The low pressure levels in children compared to the higher pressure levels in adults could be explained from the steady state formula introduced by Marmarou (1978).

The fontanelle size was also measured in the groups of newborns and infants. For this, a practical method was used, introduced by Popich and Smith (1972). The average of the width and the length of the fontanelle (mean anterior fontanelle size $=$ mafs) was measured. The value of mafs $( \pm S D)$ for the group of newborns was $2.2 \pm 0.7$ $\mathrm{cm}$ and for the group of infants $2.4 \pm 0.9 \mathrm{~cm}$. Both values are in accordance with the values reported by Popich and Smith.

For the statistical analysis the Mann-Whitney U-test, the Spearman rank correlation test and the Kruskal-Wallis test were used. A p-value $<0.05$ was considered statistically significant.

There was no statistically significant correlation between the size of the fontanelle and the level of AFP, also there was no statistically significant correlation between the fontanelle size and the quality of the AFP recording.

In chapter 5 the results of AFP measurements in 141 patients with various pediatric and neurological diseases are described. In these 141 patients 253 AFP measurements were performed. In 48 of these patients 79 continuous AFP measurements (i.e. lasting over 30 minutes) were performed. The patients were divided into diagnostic classes according to a central nervous system localisation or a non-central nervous system localisation. The ages of the patients ranged from 30 weeks gestational age to 24 months calendar age. The patients and the AFP values in each diagnostic class are represented and thoroughly discussed. All children with clinical signs of raised ICP, who had tri- or quadriventricular hydrocephalus on CT-scan had AFP values above $15 \mathrm{mmHg}$. In children with pediatric diseases usually normal AFP levels were usually found, except in children with cardiac diseases where AFP was elevated, this being probably due to venous congestion.

It was not difficult to perform continuous AFP measurements. The maximal duration was 39 hours. The average pressure level was estimated from the continuous recordings by visual inspection and by calculating the AFP level from the average of ten measuring points. Between these two methods there was no statistically significant difference. Not only the pressure level but wave patterns could also be recognized in the recordings. Lundberg (1960) described A- and B-waves, using an invasive ICP monitoring technique. Both wave patterns could also be recorded with this non-invasive method.

In 23 patients, on whom a total of 26 shunting operations or shunt revisions were performed, AFP monitoring was performed before and after the operation. The average AFP level after operation was significantly lower than before operation (12.7 \pm 4 $\mathrm{mmHg}$ versus $23.0 \pm 7.9 \mathrm{mmHg}$ ).

Sixteen comparative measurements between AFP and CSFP were performed. Both 
open and closed type measurements were done, punctures of fontanelle, ventricle, Rickham reservoir or lumbar space being performed. In children with low pressure levels $(<10 \mathrm{mmHg}$ ) the difference between AFP and CSFP was less than one $\mathrm{mmHg}$. This is not significant keeping in mind the usual measurement error $( \pm 1 \mathrm{mmHg})$. When AFP was higher $(>15 \mathrm{mmHg})$ the difference between AFP and CSFP measured with an open end manometer was greater. This is to be expected, since in the range of higher pressures there is a change from the flat part to the steep part of the volumepressure curve resulting in a greater change in pressure in non-isovolumetric measurements.

A new technique usually works in the hands of the investigator. While testing the apparatus in a clinical setting the measurements were primarily performed by the author of this thesis. Interpretation of the recordings is usually easy for the inventor and for the person who performs the recordings. However, interpretation of the recordings should be standardized to prevent observations varying among various interpreters. If the level of AFP from one recording is judged differently by various observers then the method is of no use in clinical practice (chapter 6).

It is important to realize that observer interpretation can be divided into systematic variation, which is the inter-observer variation, and non-systematic (random) variation. This random variation is caused by other sources of error of interpretation including individual (intra-observer) variation. In this study four sources of variation can be distinguished: a) patient which is the inter-patient variation, b) observer which is the inter-observer variation, c) PDC which is the intra-patient (or biological) variation, d) other variations including measurement error.

Four observers with different backgrounds, evaluated independently a randomly selected number of $82 \mathrm{AFP}$ recordings, both of normal children and of patients. During the assessment of the recordings the four observers also judged the quality of the recording and of the PDC plateau, using an ordinal five-points scale. The Wilcoxon matched pairs signed rank test tested the systematic difference between each pair of observers. The Spearman rank correlation test tested the other interpretation errors, including intra-observer variation.

The balanced study design offered the possibility of performing a variance-component analysis. Total variance (= squared standard deviation) is the sum of the following sources of variation: inter-and intra-patient variation, inter-observer variation and all other sources of interpretation errors including intra-observer variation.

From the various statistical analysis procedures it was concluded that in the interpretation of AFP recording in the group of normal children a small systematic difference was present between one observer and the other three observers. A small systematic difference was present between several observers in the case of the group of patients. The actual differences in pressure levels were very small and of no clinical relevance. The inter-observer variation as random effect in the interpretation of AFP recording was estimated to be small in both the group of normal infants and in the group of patients. The intra-observer variation was indirectly estimated as small. A substantial intra-patient (or biological) variation was present. Therefore, it was concluded that in order to characterize a patient, an average of at least three PDC's has to be taken as the AFP score of that patient. 
In order to find out whether AFP monitoring could be useful in clinical practice with respect to the question of whether or not to shunt we asked a skilled child neurologist to review the clinical data of the 141 patients of this study and to decide whether or not a patient had to be shunted (chapter 7). After this had been completed her decision was compared to the actual clinical situation (i.e. shunted or not).

In those cases where no consensus consisted $(8 \%)$ the role of AFP monitoring was evaluated in order to find out what kind of additional information could have been detected with this technique.

In chapter 7 it is pointed out that besides confirming the clinical diagnosis of raised ICP, AFP monitoring can detect early rises in ICP. It was concluded that this harmless method could be used in addition to the neurological examination and brain imaging techniques, thus adding valuable helpful information in questions of whether or not to perform shunting operations.

The overall conclusion of this thesis is that AFP monitoring with the RTT in the fontanelle adaptor is a reliable, non-invasive technique both for short term and long term ICP measurements, since application pressure does not interfere with ICP recording due to the measuring technique and the fixation method. AFP monitoring has a valuable role in the diagnosis, treatment and follow-up of young children with (suspected) intracranial pathology. 


\section{SAMENVATTING}

Sinds het introduceren van liquordrukmeting door middel van lumbaalpunctie (Quincke 1891) is de belangstelling voor de intra-craniële druk gebleven. Met het invoeren van continue intra-craniële drukmeting met een ventrikelcatheter (Lundberg 1960) werd bekend dat er specifieke golfpatronen bestaan die zowel onder normale, als onder pathologische omstandigheden kunnen voorkomen. Löfgren (1973) en Marmarou (1975) bestudeerden de relatie tussen veranderingen in volume en druk in de totale liquorruimte binnen schedelholte en wervelkanaal.

Een abnormale intra-craniële druk wijst niet per sé op pathologie. Een hoge druk $(>20 \mathrm{mmHg}$ ) kan bestaan zonder neurologische verschijnselen, bijvoorbeeld bij benigne intra-craniële hypertensie. Daarentegen kan de druk bij een ruimte-innemend proces met verschijnselen van hersenstam compressie nog normaal zijn $(<15 \mathrm{mmHg})$.

In het algemeen geldt dat bij patiënten met ernstig schedel-hersenletsel intra-craniële drukmeting is geïndiceerd wegens de daaruit voortvloeiende therapeutische consequenties. Als tweede voorbeeld kan genoemd worden de post-operatieve drukmeting na een intra-craniële ingreep. Bij kinderen zijn er bovendien specifieke indicatie-gebieden voor drukmeting: intra-ventriculaire bloeding bij prematuren of pasgeborenen, posthaemorrhagische hydrocephalus, hypoxisch-ischaemische encephalopathie, bacteriële meningitis en het syndroom van Reye.

Alle in gebruik zijnde meettechnieken waren invasief en de ontwikkelde apparatuur was weinig handzaam bij jonge kinderen. Infectiegevaar en praktische problemen deden veel clinici aarzelen om de beschikbare apparatuur bij deze groep aan te wenden. Het tot nu toe gestelde heeft betrekking op de volgende meetmethoden: liquordrukmeting door middel van punctie van lumbaalzak, cisterna magna of ventrikel; drukmeting aan het hersenoppervlak door middel van een subdurale of subarachnoïdale catheter of met behulp van een subarachnoïdale schroef; intra-parenchymateuze drukmeting met een transducer; epidurale drukmeting met diverse typen meters. Voor- en nadelen van deze methoden worden besproken in hoofdstuk 2.

Het is logisch dat is gezocht naar een niet-invasieve, handzame methode om de druk te meten. Bij pasgeborenen leent de fontanel zich daartoe. Het palperen van de fontanel is van oudsher de methode om de druk te schatten. Wentzler (1922) introduceerde een tonometer met vaste gewichtjes als fontaneldrukmeter. In 1959 modificeerden Davidoff en Chamlin de oogboldrukmeter tot 'fontanometer'. Deze instrumenten bleken niet praktisch in het gebruik en gaven de fontaneldruk niet nauwkeurig genoeg weer. De methode waarbij gebruik werd gemaakt van op de fontanel bevestigde ballonnetjes, gevuld met lucht of vloeistof (Purin 1964, Brett 1966, Blaauw 1974), bleek onbetrouwbaar en technisch moeilijk uitvoerbaar. Sinds 1977 zijn diverse soorten fontanelmeters ontwikkeld gebaseerd op het principe van de 'pneumatic flow switch' (Numoto 1966). Van al dit soort meters is de 'Ladd fiber-optic meter' populair geworden (Hill en Volpe 1981). Ook het principe van de Wheatstone brug bleek bruilkbaar om fontaneldrukmeters te ontwerpen (Wealthall en Smallwood 1974, Huch 1979). De twee laatstgenoemde methoden werden tot dusver beschouwd als het meest betrouwbaar voor het meten van de fontaneldruk. 
Bij alle bovengenoemde methoden blijkt de fixatie van de meters het grootste probleem te zijn. Het meetinstrument moet voldoende stevig worden bevestigd zonder dat de applicatiedruk het meetresultaat beïnvloedt.

Holtedrukmeting door een intacte membraan, gebaseerd op het co-planariteitsprincipe, werd toegepast in de verloskunde (Smyth 1957) en in de oogheelkunde (Mackay 1964). Om de druk door een intacte en flexibele membraan te kunnen meten is het noodzakelijk dat de krachten in de membraan worden geëlimineerd of tenminste sterk verminderd. Dit kan worden bereikt door aplanatie en co-planariteit. Hierbij wordt de membraan over een bepaald gedeelte vlak gemaakt, met als gevolg dat de trekkrachten in de membraan tot vrijwel nul worden gereduceerd.Mackay (1964) beschreef als eerste een karakteristieke curve bij het co-planair aanbrengen van een oogboldrukmeter. Reeds in 1964 wees Mackay op de mogelijkheid dit principe aan te wenden voor fontaneldrukmeting.

Voor de neurologie werd het co-planariteitsprincipe voor het eerst toegepast door Schettini (1974) bij epidurale drukmeting op de intacte dura. De meetresultaten werden grafisch weergegeven in de zogeheten druk-diepte curve ('pressure depth curve' of 'PDC'). In dit proefschrift speelt de PDC bij fontaneldrukmeting een essentiële rol.

In het Academisch Ziekenhuis Rotterdam - Dijkzigt is een implanteerbare telemetrische drukmeter ontwikkeld door De Jong (1975) voor het meten van epidurale druk bij patiënten met gesloten schedelnaden. In hoofdstuk 3 wordt deze Rotterdam Teletransducer (RTT) beschreven.

Er bestaan grote overeenkomsten tussen het meten van de epiduraaldruk en de fontaneldruk. Bij epiduraal drukmeten wordt de transducer direct op de dura geplaatst, bij fontaneldrukmeting bevinden zich als extra structuren huid en onderliggend weefsel tussen de transducer en de dura.

$\mathrm{Bij}$ fontaneldrukmeting vormt de applicatiedruk een probleem op zich. Om dat probleem op te lossen is een speciale adaptor ontworpen die de mogelijkheid biedt om de RTT nauwkeurig op diepte in te stellen. Deze adaptor moet zorgvuldig op de benige fontanelbegrenzing worden geplaatst, een soepel siliconen frame dient ertoe de adaptor te fixeren. Op deze wijze wordt eventueel benodigde applicatiedruk afgeleid via de benige schedel. Een flexibele meetkabel tussen de adaptor en de 'bedside-monitor' geeft het kind voldoende bewegingsvrijheid tijdens de meetprocedure.

$\mathrm{Na}$ het plaatsen en fixeren van de RTT volgt de ijkprocedure. Bij aanvang van de meting bevindt de RTT zich in de adaptor boven de huid. Met een langzaam ronddraaiende beweging wordt de transducer naar de huid gebracht. Voordat deze de huid raakt (er worden dan nog geen polspulsaties geregistreerd) worden de pennen van de recorder op nul gesteld voor respectievelijk druk en insertie diepte. Door de transducer, draaiend met de hand, dieper in te brengen verschijnt er een karakteristieke druk-diepte curve op het papier. Aanvankelijk stijgt de druk, waarna een vlak stuk of 'plateau' wordt bereikt waarop de polspulsaties gelijk blijven. De transducer doorkruist hier de lege subarachnoïdale ruimte en ontmoet geen extra tegenkrachten, derhalve wordt er op dit moment geen extra toename van de druk geregistreerd. De 'plateau-druk' die nu wordt geregistreerd is gelijk aan de intra-craniële druk. Als de transducer dieper wordt gesteld, stijgt de druk snel ten gevolge van compressie van de subarachnoïdale ruimte en het onderliggend hersenweefsel. Door het terugdraaien 
van de transducer volgt een druk-diepte curve in spiegelbeeld. Met deze methode kunnen zowel kortdurende als langdurende (continue) fontaneldrukmetingen worden uitgevoerd. Voor continue fontaneldrukmeting wordt de transducer gesteld op de diepte overeenkomend met het plateau van de druk-diepte curve.

Om de fontaneldruk bij een patiëntje vast te stellen wordt driemaal een druk-diepte curve gemaakt. Iedere druk-diepte curve bevat twee plateaus. Het gemiddelde van de aldus verkregen zes plateaus is de fontaneldruk.

Om normaalwaarden van de fontaneldruk te verkrijgen werd bij gezonde pasgeborenen en zuigelingen de druk gemeten. Dit wordt beschreven in hoofdstuk 4. De gemiddelde fontaneldruk van 30 pasgeborenen met een leeftijd van 0 tot 9 dagen, verdeeld in klassen van 2 dagen, was $8.9 \pm 3.0 \mathrm{mmHg}$. De gemiddelde druk van 52 zuigelingen met een leeftijd van 30 tot 330 dagen, verdeeld in klassen van 60 dagen, was 11.0 $\pm 1.9 \mathrm{mmHg}$. In beide groepen kon geen statistisch significant verschil tussen de verschillende leeftijdsklassen worden aangetoond. De genoemde normaalwaarden komen overeen met literatuurgegevens.

De gemiddelde fontaneldruk in de groep van 30 pasgeborenen verschilde statistisch significant van de gemiddelde druk in de groep van 52 zuigelingen. Dit leeftijdsafhankelijk drukverschil is ook door anderen beschreven. Waarom de druk bij pasgeborenen lager is dan bij oudere kinderen en bij oudere kinderen weer lager dan bij volwassenen is niet bekend. Een mogelijke verklaring kan volgen uit de steady-state formule, die in 1978 werd geïntroduceerd door Marmarou. De liquorproduktie zou bij kinderen iets lager zijn dan bij volwassenen, resulterend in een lagere waarde van de druk. In hoofdstuk 4 wordt een overzicht gegeven van de literatuur over normale waarden van intra-craniële druk bij kinderen, gemeten met niet-invasieve en invasieve technieken.

De fontanelgrootte bij de gemeten kinderen is uitgedrukt als de som van lengte en breedte, gedeeld door twee (Popich en Smith 1972). Bij pas-geborenen was deze 'mean anterior fontanelle size' $2.2 \pm 0.7 \mathrm{~cm}$, bij zuigelingen $2.4 \pm 0.9 \mathrm{~cm}$. Deze gegevens wijken niet af van de waarden die Popich en Smith vonden.

Voor de statistische analyses werd gebruik gemaakt van de Mann-Whitney U-test, de Spearman rank correlation test en de Kruskal-Wallis test. Een p-waarde $<0.05$ werd als statistisch significant beschouwd. De hoogte van de fontaneldruk bleek niet door de grootte van de fontanel te worden beïnvloed. Bij het beoordelen deed het er niet toe of de fontanel groot of klein was.

In hoofdstuk 5 worden de resultaten beschreven van fontaneldrukmetingen bij 141 patiënten. Bij deze patiënten, met verschillende pediatrische en neurologische ziektebeelden, werden 253 metingen verricht. $\mathrm{Bij} 48$ van deze patiënten werden 79 continue drukmetingen uitgevoerd, waarvan de langste meting 39 uur duurde.

De patiënten zijn ingedeeld in twee diagnostische categoriën gebaseerd op het al dan niet bestaan van een aandoening van het centrale zenuwstelsel. De leeftijd van de patiënten varieerde van 30 weken prematuritas tot 24 maanden kalenderleeftijd. De patiënten en de fontaneldrukwaarden in elke diagnostische categorie worden vermeld en uitvoerig besproken. Bij alle kinderen met klinische verschijnselen van verhoogde intra-craniële druk, die bij CT-onderzoek een tri- of quadriventriculaire hydrocephalus 
hadden, was de fontaneldruk meer dan $15 \mathrm{mmHg}$. Bij kinderen met pediatrische (niet neurologische) ziektebeelden werden gewoonlijk normale waarden voor de fontaneldruk gevonden, behalve wanneer er sprake was van ziekten van hart of bloedvaten. Bij laatstgenoemden was de fontaneldruk meestal verhoogd, waarschijnlijk als gevolg van veneuze stuwing.

Een aparte paragraaf is gewijd aan de continue drukmetingen. Het bepalen van de fontaneldruk geschiedt hier enigszins anders dan bij de kortdurende metingen. Het gemiddelde drukniveau werd allereerst visueel geschat en daarna gemeten door het gemiddelde van 10 vaste meetpunten te bepalen. Tussen deze twee beoordelingsmethoden bleek geen statistisch significant verschil te bestaan.

De door Lundberg (1960) met behulp van de invasieve methode gevonden A- en B-golven bleken ook met de hier beschreven niet-invasieve methode aantoonbaar.

Bij 23 patiënten ( 26 drainoperaties) werd de fontaneldruk gemeten voor en na operatie. De gemiddelde post-operatieve druk was significant lager dan de pre-operatieve druk $(12.7 \pm 4.0 \mathrm{mmHg}$ versus $23.0 \pm 7.9 \mathrm{mmHg})$.

Zestien keer werd een meting uitgevoerd om de fontaneldruk met de liquordruk te kunnen vergelijken. Deze metingen werden uitgevoerd, zowel met een open als met een gesloten manometer. Doordat de volume-druk curve in het traject van de lage druk min of meer lineair is en bij hogere druk exponentieel stijgt, zolang het meetsysteem gesloten blijft, is het meten met een open systeem af te raden. De hierbij gevonden waarden zijn bij lage druk nog wel betrouwbaar maar bij hogere druk neemt deze betrouwbaarheid af. Bij de in deze dissertatie beschreven vergelijkende metingen werd dit bevestigd (hoofdstuk 5).

Ervaring speelt bij iedere techniek een belangrijke rol. Bij het uittesten van de RTT werden de metingen voornamelijk uitgevoerd door de schrijfster van dit proefschrift. Het beoordelen van registraties is meestal eenvoudig voor degene die het meetinstrument heeft ontwikkeld en voor degenen die de metingen uitvoeren. Om 'observer' variatie te voorkomen werden de registraties op een gestandaardiseerde manier beoordeeld (hoofdstuk 6). In deze studie kunnen vier variatiebronnen worden onderscheiden: (a) de patiënt of inter-patiënt variatie, (b) de observer of inter-observer variatie. (c) de PDC, en (d) andere variaties waaronder meetfouten.

Vier observers, elk met een andere achtergrond beoordeelden, onafhankelijk van elkaar, een a-select getrokken antal van 82 fontaneldrukmetingen uit zowel de groep normale kinderen als uit de groep patiënten. $\mathrm{B} \ddot{j}$ het bepalen van de fontaneldruk keken de observers naar de plateaus van de eerste drie druk-diepte curves en tevens, gebruik makend van een ordinale vijfpunts- schaal naar de kwaliteit van de registraties.

Met behulp van de Wilcoxon matched pairs signed rank test werden eventuele systematische verschillen tussen twee observers bepaald. Met de Spearman rank correlation test werd nagegaan of er andere interpretatiefouten waren. De gebalanceerde opzet van de studie verschafte de mogelijkheid een variantie-componenten analyse uit te voeren. De totale variantie $\left(\mathrm{SD}^{2}\right)$ is de som van de volgende variatiebronnen: inter- en intra-patiënt variatie, inter-observer variatie en alle andere bronnen van interpretatiefouten, waaronder ook de intra-observer variatie.

$\mathrm{Bij}$ het beoordelen van de fontaneldrukmetingen in de groep normale kinderen bleek tussen één observer en de andere drie een klein systematisch verschil in interpretatie 
van de registraties te bestaan. Tussen de vier verschillende observers werd bij het beoordelen van de registraties van de patiëntengroep eveneens een miniem systematisch verschil gezien. Deze verschillen in druk waren echter zeer klein en zonder klinische betekenis. De random inter-observer variatie werd, zowel in de groep normale kinderen, als in de patiëntengroep, laag geschat. De intra-observer variatie werd op indirecte wijze geschat en bleek eveneens laag. Er was een vrij grote intra-patiënt variatie. Daarom werd geconcludeerd dat tenminste drie druk-diepte curven moeten worden geanalyseerd om per patiënt de gemiddelde fontaneldruk te berekenen.

De klinische relevantie van fontaneldrukmeting werd nagegaan door een ervaren kinderneurologe op grond van een ontslagbrief te laten bepalen of een drainoperatie wel of niet was geindiceerd. Vervolgens werd haar mening vergeleken met de feitelijke klinische situatie waarin de betreffende patiënt wel of geen drain had gekregen. Daarna werd gekeken welke aanvullende informatie de fontaneldrukmeting zou hebben opgeleverd, met name in die gevallen waarbij de mening van de kinderneurologe afweek van die van de clinici die de patiënt hadden behandeld. In hoofdstuk 7 wordt een en ander toegelicht. Geconcludeerd werd dat fontaneldrukmeting niet alleen de klinische diagnose van verhoogde intra-craniële druk bevestigt, maar dat met deze methode ook een vroege stijging in de intra-craniële druk kan worden opgespoord alvorens er klinische verschijnselen optreden. Deze niet-invasieve techniek kan worden gebruikt als extra diagnostisch hulpmiddel naast het neurologisch onderzoek en CT-scan of echo van de schedel en levert waardevolle anvullende informatie die belangrijk is bij de vraag of er wel of geen drain moet worden geplaatst.

De algemene conclusie van dit proefschrift is dat de intra-craniële druk bij kinderen met een open fontanel betrouwbaar kan worden gemeten met de Rotterdam Teletransducer. Door de speciale fontanel-adaptor en het fixatieframe wordt het meetresultaat niet beïnvloed door de applicatiedruk. De methode is geschikt voor zowel kort- als langdurende metingen. Fontaneldrukmeting levert een waardevolle bijdrage aan de diagnostiek, behandeling en follow-up van jonge kinderen met (verdenking op) intra-craniële pathologie. 


\section{REFERENCES}

Adams RD, Lyon G (1982). Neurology of hereditary metabolic diseases of children. McGraw-Hill Book Company, New York, 936.

Ahmann PA, Lazarra A, Dykes FD, Brann AW, Schwartz JF (1980). Intraventricular hemorrhage in the high-risk preterm infant: incidence and outcome. Ann Neurol 7: 118-124.

Aisenson MR (1950). Closing of the anterior fontanelle. Pediatrics 6: 223-225.

Allen R (1986). Intracranial pressure: a review of clinical problems, measurement techniques and monitoring methods. J Med Eng Technol 10: $299-320$.

Alvarez LA, Maytal J, Shinnar S (1986). Idiopathic external hydrocephalus: natural history and relationship to benign familial macrocephaly. Pediatrics 77: 901-907.

Arnold H, Laas R (1980). Plateau waves: production in the rat and simulation by means of a mechanical model. In: Shulman K, Marmarou A, Miller JD, Becker DP, Hochwald GM, Brock M (ed). Intracranial pressure IV. Springer, Berlin Heidelberg New York, 525-529.

Atkinson JR, Shurtleff DB, Foltz EL (1967). Radiotelemetry for the measurement of intracranial pressure. J Neurosurg 27: 428-432.

Auer LM, Sayama I (1983). Intracranial pressure oscillations (B-waves) caused by oscillations in cerebrovascular volume. Acta Neurochir 68: 93-100.

Avezaat CJJ, Eijndhoven JHM van (1984). Cerebrospinal fluid pulse pressure and craniospinal dynamics. Erasmus University Rotterdam. Jongbloed A \& son (ed), The Hague, The Netherlands. Thesis, pp 339.

Bada HS (1983). Intracranial monitoring. Its role and application in neonatal intensive care. Clin Perinat 10: 223-236.
Baerts W (1984). Intracranial Hemorrhage in preterm infants, an ultrasound study. Erasmus University Rotterdam, Kanters, Alblasserdam, The Netherlands. Thesis, pp 239.

Barashnev YI, Leontiev AF (1966). Cerebrospinal fluid pressure in premature infants with and without intracranial birth injury; abstracted by Crome L. Dev Med Child Neurol 8: 117.

Barlow P, Mendelow AD. Rowan JO, Lawrence A, Barlow M (1986). Clinical evaluation of the Gaeltec ICT/b pressure transducer placed subdurally. In: Miller JD, Teasdale GM, Rowan JO, Galbraith SL, Mendelow AD (ed). Intracranial Pressure VI. Springer, Berlin Heidelberg New York Tokyo, 181-183.

Beks JWF, Alberda S, Gieles ACM, Kuypers MH, Flanderijn H (1977). Extradural transducer for monitoring intracranial pressure. Acta Neurochir 38: 245-250.

Blaauw G, van der Bos JL, Mus A (1974). On pulsations of the fontanelle. Dev Med Child Neurol 16, suppl 32:23-26.

Blomquist HKS, Sundin S, Ekstedt J (1986). Cerebrospinal fluid hydrodynamic studies in children. J Neurol Neurosurg Psych 49: 536548.

Börgesen SE, Gjerris F (1982). The predictive value of conductance to outflow of CSF in normal pressure hydrocephalus. Brain 105: 6586.

Boston VE, Hofmann DA, Scott JES (1976). The mechanical aplanation tonometer for the non-invasive measurement of intracranial pressure in infants. $Z$ Kinderchir 19: 121-128.

Bresnan MJ, Lorenzo AV (1975). Cerebrospinal fluid dynamics in megalencephaly. Dev Med Child Neurol 17, suppl 35: 51-57. 
Brett EM (1966). Measurement of cerebrospinal fluid pressure in infants without puncture. Dev Med Child Neurol 8: 207-210.

Brett EM (1983). Pediatric Neurology. Churchill Livingstone, Edinburgh London Melbourne New York, 385-404.

Brock M, Diefenthäler K (1972). A modified equipment for the continuous telemetric monitoring of epidural or subdural pressure. In: Brock M, Dietz H (ed). Intracranial pressure. Springer, New York Heidelberg Berlin, 21-26.

Brock M (1983). ICP revisited. In: Ishii S, Nagai $H$, Brock M (ed). Intracranial pressure V. Springer, Berlin Heidelberg, 3-7.

Brömme W, Baum WF, Hirsch W, Schaps P, Schobess A (1985). Methode zur nicht-invasiven Messung und Registrierung des intrakraniellen Druckes (ICP) über die offenen Fontanelle des Säuglings. Zb1 Neurochir 46: 159170.

Bruce DA, Schut L, Bruno LA (1976). The role of intracranial pressure monitoring in a pediatric intensive care unit. In: Beks JWF, Bosch DA, Brock M (ed). Intracranial pressure III. Springer, Berlin Heidelberg New York, 323329.

Bruce DA, Raphaely RC, Goldberg AI, Zimmerman RA, Bilaniuk LT, Schut L, Kuhl DE (1979). Pathofysiology, treatment and outcome following severe head injury in children. Child's Brain 5: 174-191.

Bunegin L, Albin MS, Rauschhuber R, Marlin $\mathrm{AE}$ (1987). Intracranial pressure measurement from the anterior fontanelle utilizing a pneumo-electronic switch. Neurosurgery 20: 726-731.

Chapman PH, Zervas NT, Cosman ER (1980). A device for telemetric pressure measurement in shunt systems. In: Shulman K, Marmarou A, Miller JD, Becker DP, Hochwald GM, Brock M (ed). Intracranial Pressure IV. Springer, Berlin Heidelberg New York, 489491.

Clar HE, Roosen C, Brenner A (1981). Longterm results after drainage of subdural effusions in infants. $Z$ Kinderchir 34: 164-166.
Coe JE, Nelson WJ, Rudenberg FH, Garza $\mathbf{R}$ (1967). Technique for continuous intracranial pressure recording. Technical note. J Neurosurg 27: 370-375.

Coroneos NJ, Turner JM, Gibson RM, McDowall DG, Pickerodt VWA, Keaney NP (1972). Comparison of extradural with intraventricular pressure in patients after head injury. In: Brock M, Dietz H (ed). Intracranial pressure. Springer, Berlin Heidelberg New York, 51-58.

Coroneos NJ, McDowall DG, Gibson RM, Pickerodt VWA, Keaney NP (1973). Measurement of extradural pressure and its relationship to other intracranial pressures. J Neurol Neurosurg Psychiatry 36: 514-522.

Cosman ER, Zervas NT, Chapman PH, Cosman BJ, Arnold MA (1979). A telemetric pressure sensor for ventricular shunt systems. Surg Neurol 11: 287-294.

Crevel H van (1982). Pathogenesis of raised cerebrospinal fluid pressure. Doc Ophthalmol 52: 251-257.

Cutler RWP, Page LK, Galicich J, Watters GV (1968). Formation and absorption of cerebrospinal fluid in man. Brain 91: 707-720.

Daniels HU, Pothmann R, Kauther K, Lim $D P$ (1981). The aplanation tonometry of the fontanelle : A non-invasive instrument for the management of hydrocephalus in infancy. $Z$ Kinderchir 34: 144-146.

Davidoff LM, Chamlin M (1959). The 'fontanometer'. Adaptation of the Schiotz tonometer for the determination of intracranial pressure in the neonatal period and early period of infancy. Pediatrics 24: 1065-1068.

Dean JM, McComb JG (1981). Intracranial pressure monitoring in severe pediatric neardrowning. Neurosurgery 9: 627-630.

DeMyer W (1972). Megalencephaly in children. Neurology 22: 634-643.

DeMyer W (1986). Megalencephaly: Types, Clinical Syndromes and Management. Pediatr Neurol 2: 321-328. 
Dearden NM, McDowall DG, Gibson RM (1984). Assessment of Leeds device for monitoring intracranial pressure. $\mathrm{J}$ Neurosurg 60: 123-129.

Devictor D, Checoury A, Petiot A, Benichon JJ, Huault G (1983). Hypertension intra-crânienne dans les méningites à pneumococques. Arch Fr Pediatr 40: 715-717.

Dietrich K, Gaab MR, Knöblich OE, Schupp J, Ott B (1977). A new miniaturized system for monitoring the intracranial pressure in children and adults. Neuropädiatrie 8: 21-28.

Dietze R, Meyer R, Vesper J (1972). Über Pulsationsmessung und - darstellung an der offenen Fontanella major. Psych Neurol Med Psych 24: 35-40.

DiRocco C, McLone DG, Shimoji T, Raimondi AJ (1975). Continuous intraventricular cerebrospinal fluid pressure recording in hydrocephalic children during wakefulness and sleep. J Neurosurg 42: 683-689.

Donn SM, Philip AGS (1978). Early increase in intracranial pressure in preterm infants. Pediatrics 61: 904-907.

Dorsch NWC, Stephens R, Symon L (1971). An intracranial pressure transducer. Biomed Eng 6: 452-457.

Dorsch NWC, Symon L (1975). A practical technique for monitoring extradural pressure. J Neurosurg 42: 249-257.

Duggins EL, LeRoy PL, Goloskow JW (1978). Pneumatic applanation as a non-invasive means of intraventricular pressure measurement. J Neurosurg Nurs 10: 156-159.

Durham DG, Bigliano RP, Masino JA (1965). Pneumatic applanation tonometer. Tram Acad Opth Otol 6: 1029-1047.

Dykes FD, Dunbar B, Lazarra A, Ahman PA (1989). Posthemorrhagic hydrocephalus in highrisk preterm infants: natural history, management and long-term outcome. J Pediatr 114: 611-618.

Easa D, Tran A, Bingham W (1983). Noninvasive intracranial pressure measurement in the newborn. An alternate method. Am J Dis Child 137: 332-335.
Edwards J (1974). An intracranial pressure tonometer for use on neonates: preliminary report. Dev Med Child Neurol 16, suppl 32: 38-39.

Emery JR, Peabody JL (1983). Head position affects intracranial pressure in newborn infants. J Pediatr 103: 950-953.

Fisher DM, Frewen T, Swedlow DB (1982). Increase in intracranial pressure during suctioning - stimulation vs rise in $\mathrm{Pa} \mathrm{CO} 2$. Anaesthesiology 57: 416-417.

Fleischer AS, Payne NS, Tindall GT (1976). Continuous monitoring of intracranial pressure in severe closed head injury without mass lesions. Surg Neurol 6: 31-34.

Forrest DM, Tsingoglou S (1968). The false fontanelle as a practical method of longterm testing of intracranial pressure. Dev Med Child Neurol 16: 17-20.

Fryer ThB, Corbin SD, Silverberg GD, Schmidt EV, Ream AK (1978). Telemetry of intracranial pressure. Biotelemetry Patient Monitg 5: 88-112.

Furuse M, Hasuo M, Kuchiwaki H, Ikeyama A, Inagaki H, Ishihara T, Igarashi I, Kitano $T$ (1983). An indwelling epidural pressure sensor with integxated circuits for biotelemetry. In: Ishii S, Nagai H, Brock M (ed). Intracranial Pressure V. Springer, Berlin Heidelberg New York, 90-94.

Gaab MR, Sörensen N, Brawanski A, Bushe KA, Wodarz R (1980). Non-invasive intracranial pressure monitoring by fontanometry. $\mathrm{Z}$ Kinderchir 31: 339-347.

Gaab MR, Sörensen N, Hufenbeck B (1980/ 81). Fontanometrie zur nicht invasiven Registrierung des intrakraniellen Druckes. Pädiat Prax 24: 631-644.

Gaab MR, Sörensen N, Brawanski A, Haubitz I (1982). Value of ICP monitoring in infancy and childhood. Monogr Paediatr 15: $124-130$.

Gaab MR (1984). Die Registrierung des Intrakraniellen Druckes. Fortschr Med 102: 957962. 
Gaab MR, Ungersböck $K$, Hufenbeck B (1986). Evaluation of ICP by computerized bedside monitoring: methods and clinical significance. Neurol Res 8: 44-52.

Gerlach J, Jensen HP, Koos W, Kraus H (1967). Pädiatrische Neurochirurgie. Thieme Verlag, Stuttgart, 138-139.

Gobiet W, Bock WJ, Liesegang J, Grote W (1972). Long-time monitoring of epidural pressure in man. In: Brock $M$, Dietz $H$ (ed). Intracranial Pressure. Springer, Berlin, 14-17.

Goitein KJ, Amit Y, Mussaffi H (1983). Intracranial pressure in central nervous system infections and cerebral inschaemia of infancy. Arch Dis Child 58: 184-186.

Golabi M, Edwards MSB, Ousterhout DK (1987). Craniostenosis and hydrocephalus. Neurosurgery 21: 63-67.

Gooskens RH, Willemse J, Gielen C (1985). Cerebrospinal fluid dynamics and cerebrospinal fluid infusion in children: Part II. Clinical application of lumbar cerebrospinal fluid infusion in children with macrocephaly and a normal growth rate of the head circumference. Neuropaediatrics 16: 121-126.

Gooskens RHJM (1988). Megalencephaly, a subtype of macrocephaly. Thesis, University of Utrecht, Addix, Wijk bij Duurstede, pp 157.

Gorlin RJ (1987). Nevoid basal cell carcinoma syndrome. In: Gomez MR (ed). Neurocutaneous diseases. Butterworths, Boston London Sydney, 67-79.

Grant DN (1971). Benign intracranial hypertension. Arch Dis Child 46: 651-655.

Gücer G, Viernstein LJ, Chubbuck JG, Walker AE (1979). Clinical evaluation of long-term epidural monitoring of intracranial pressure. Surg Neurol 12: 373-377.

Gücer G, Viernstein LJ, Walker AE (1980). Continuous intracranial pressure recording in adult hydrocephalus. Surg Neurol 13, 323-328.

Guillaume J, Janny P (1951). Manométrie intracranienne continue. Rev Neurol 84: 131142.
Haase J (1986). Germinal matrix haemorrhage (GMH) syndrome and intraventricular haemorrhage (IVH). Syndrome in the newborn. In: Raimondi AJ, Choux M, Di Rocco C (ed). Heads injuries in the newborn and infant. Springer, New York Heidelberg London Paris Tokyo, 111-124.

Hamer JPM, van Wijk LM, Lie KI (1986). Het Kukelfenomeen; klinische waarde en oorsprong van de terminologie. Hart Bulletin 17: 111-113.

Harbaugh RE, Saunders RL (1984). The false fontanel. J Neurosurg 61: 71-75.

Hayashi T, Watanabe M, Kuramoto S (1978). Measurement of intracranial pressure via the anterior fontanelle. Kurume Med J 25: 141-150.

Hayden PW, Foltz EL, Shurtleff DB (1968). Effect of an oral osmotic agent on ventricular fluid pressure of hydrocephalic children. Pediatrics 41: 955-967.

Hayden PW, Shurtleff DB, Foltz EL (1970). Ventricular fluid pressure recordings in hydrocephalic patients. Arch Neurol 23: 147-154.

Heppner F, Lanner G, Rodler H (1976). Telemetry of intracranial pressure. Acta Neurochir 33: 37-43.

Hill A, Volpe JJ (1981a). Measurement of intracranial pressure using the Ladd intracranial pressure monitor. J Pediatr 98: 974-976.

Hill A, Volpe JJ (1981b). Normal pressure hydrocephalus in the newborn. Pediatrics 68: 623-629.

Hill A (1983). Ventricular dilatation following intraventricular hemorrhage in the premature infant. Can J Neurol Sc 10: 81-85.

Hirsch JF, Lacombe J, Pierre-Kahn A, Renier $D$ (1978). Mesure de la pression intracranienne par palpeur de fontanelle. Neurochirurgie 24: 89-93.

Hoffman HJ (1986). How is pseudotumor cerebri diagnosed ? Arch Neurol 43: 167-168.

Holbrook PR (1978). Non-invasive measurement of intracranial pressure: Evaluation of a technique. Ped Res 12: 552. 
Hollingsworth D, Fridlund P, Vos H, Daily EK (1988). Use of fiber-optic pressure transducer for intracranial pressure measurements: a preliminary report. Heart \& Lung: The journal of critical care 17: 111-118.

Honda E, Hayashi T, Kuramoto S, Shyojima $\mathrm{T}$, Anegawa S, Ishibashi A, Ohshima Y, Nakashima O (1982). Non-invasive measurement of ICP via the anterior fontanelle by miniaturized transducer. Monogr Paediatr 15: 12-16.

Hoppenstein R (1965). A device for measuring intracranial pressure. Lancet I: 90-91.

Horbar JD, Yeager S, Philip AGS, Lucey JF (1980). Effect of application force on noninvasive measurement of intracranial pressure. Pediatrics 66: 455-457.

Horbar JD, Lucey JF, Yeager SB, Philip AGS (1981). Measurement of intracranial pressure. Pediatrics 67:74.

Huch A, Fallenstein F, Huch R, Peabody JL (1979). A new device for non-invasive measurement of intracranial pressure (ICP) in newborns (technical note). Med Progr Technol 6: 185187.

Hulme A, Cooper R (1966). A technique for the investigation of intracranial pressure in man. J Neurol Neurosurg Psychiatry 29: 154-156.

Ivan LP, Choo SH, Ventureyra ECG (1980). Intracranial pressure monitoring with the fiber optic transducer in children. Child's Brain 7 : 303-313.

Ivan LP, Badejo A (1983). Clinical and experimental observations with fontanel pressure measurements. Child's Brain 10: 361-368.

Jacobson SA, Rothballer AB (1967). Prolonged measurement of experimental intracranial pressure using a subminiature absolute pressure transducer. J Neurosurg 26: 603-608.

James HE, Bruno L, Schut L, Shalna E (1975). Intracranial pressure monitoring with a subarachnoid bolt in children. Surg Neurol 3: 313315.
James HE, Bruno L, Shapiro H, Levitt JD, Aidinis S, Langfitt TW, Shalna E (1976). Methodology for intraventricular and subarachnoid continuous recording of intracranial pressure in clinical practice. Acta Neurochir 33: 4551.

Jennett B (1977). Raised intracranial pressure. Measurement of ICP. In: An introduction to neurosurgery, 3rd edition. William Heinemann medical books Limited, London, 8-9.

Jong DA de, Ouden AH den, Boon A van de, Eijskoot F, Maas AIR (1975). Telemetered Epidural Pressure. Biotelemetry 2: 257-264.

Jong DA de, Berfelo MW, Lange SA de, Maas AIR (1979). Epidural Pressure Monitoring with the so-called Rotterdam Transducer. Further in vivo results. Acta Neurochirurgica 45: 301309.

Jong DA de, Maas AIR, Berfelo MW, Ouden AH den, Lange SA de (1982). The Rotterdam Teletransducer: A telemetric device for measuring epidural pressure. Biotelemetry Patient Monitg 9: 154-165.

Jong DA de, Maas AIR, Ouden AH den, Lange SA de (1983/1984). Long term intracranial pressure monitoring. Med Progr Technol 10: 89-96.

Jong DA de, Maas AIR, Voort E van der (1984). Non-invasive intracranial pressure monitoring. A technique for reproducible fontanelle pressure measurements. $Z$ Kinderchir 39: 274-276.

Jörgensen PB, Riishede J (1972). Comparative clinical studies of epidural and ventricular pressure. In: Brock M, Dietz $\mathrm{H}$ (ed). Intracranial pressure. Springer, Berlin Heidelberg New York, 41-45.

Kaiser AM, Whitelaw AGL (1985). Cerebrospinal fluid pressure during post haemorrhagic ventricular dilatation in newborn infants. Arch Dis Child 60: 920-924.

Kaiser AM, Whitelaw AGL (1986). Normal cerebrospinal fluid pressure in the newborn. Neuropediatrics 17: 100-102. 
Kaiser AM, Whitelaw AGL (1987a). Noninvasive monitoring of intracranial pressureFact or Fancy. Dev Med Child Neurol 29: 320326.

Kaiser AM, Whitelaw AGL (1987b). Intracranial pressure estimation by palpation of the anterior fontanelle. Arch Dis Child 62: 516-517.

Kaiser G, Minnikus H (1985). Simultaneous measurement of ventricular fluid pressure and fontanelle pressure in neonates with hydrocephalus. Z Kinderchir 40: 3-6.

Kendall B, Holland I (1981). Benign communicating hydrocephalus in children. Neuroradiology 21: 93-96.

Kinal ME (1962). Hydrocephalus and the dural venous sinuses. J Neurosurg 19: 195-201.

Kindt W (1975). Simplification of intracranial pressure monitoring. In: Lundberg N, Ponten U, Brock M (ed). Intracranial pressure II. Springer, Berlin Heidelberg New York, 381-383.

Kleiber M (1945). A tympanometer. Rev Scient Instr, vol.16(4): 79-81.

Kosteljanetz M, Borgesen SE, Stjernholm P, Christensen L, Osgaard O, Gjerris F, Rosenorn J (1986). Clinical evaluation of a simple epidural pressure sensor. Acta Neurochir 83: 108-111.

Koster WG, Kuypers MH (1980). Intracranial pressure and its epidural measurement. Med Progr Technol 7, 21-27.

Kuramoto S, Moritaka K, Hayashi T, Honda E, Shojima T (1986). Non-invasive mesaurement in intracranial pressure and analysis of the pulsewave form. Neurol Res 8: 93-96.

Landy HJ, Villanueva PA (1984). An improved subarachnoid screw for intracranial pressure monitoring. Technical note. J Neurosurg 61: 606-608.

Lange SA de, Denier van der Gon JJ, Vlieger M de (1968). Preliminary study on the ventricular pressure in hydrocephalic patients. Psychiat Neurol Neurochir 71: 267-273.
Lange SA de, Vlieger M de (1970). Hydrocephalus associated with raised venous pressure. Dev Med Child Neurol, suppl 22: 28-32.

Lange SA de, Jong DA de, Maas AIR (1978). Experience with the so-called Rotterdam Epidural Teletransducer. Z Kinderchir. 22: 425429.

Levene MI, Evans DH (1983). Continuous measurement of subarachnoid pressure in the severely asphyxiated newborn. Arch Dis Child 58: 1013-1015.

Levene MI, Evans DH (1985). Medical management of revised intracranial pressure after severe birth asphyxia. Arch Dis Child 60: 1216.

Levene MI, Evans DH, Forde A, Archer LNJ (1987). Value of intracranial pressure monitoring of asphyxiated newborn infants. Dev Med Child Neurol 29: 311-319.

Levin AB (1977a). The use of a fiber optic intracranial pressure monitor in clinical practice. Neurosurg 1: 266-271.

Levin AB (1977b). The use of a fiber optic intracranial pressure transducer in the treatment of head injuries. J Trauma 17: 767-774.

Levin AB, Kahn AR, Bahr D (1983a). Epidural intracranial pressure monitoring: a new method. In: Ishii S, Nagai H, Brock M (ed). Intracranial pressure V. Springer, Berlin Heidelberg New York, 85-89.

Levin AB, Kahn AR, Bahr D (1983b). Epidural intracranial pressure monitoring: a new system. Med Instr 17: 293-296.

Levinson A (1928). Cerebrospinal fluid in infants and in children. Am J Dis Child 36: 799818.

Leyden E (1866). Beiträge und Untersuchungen zur Physiologie und Pathologie des Gehirns, Ueber Hirndruck und Hirnbewegungen. Arch für Pathologische Anatomie und Physiologie und für Klinische Medizin 37: 519-559.

Lindhout D (1985). Teratogenesis in maternal epilepsy. New aspects of prevention. Thesis, Free University of Amsterdam, The Netherlands. 
Löfgren J, Essen C von, Zwetnow NN (1973). The pressure-volume curve of the cerebrospinal fluid space in dogs. Acta Neurol Scand 49: $557-$ 574.

Löfgren J (1975). Mechanical basis of the CSF pressure volume curve. In: Lundberg N, Pontén U, Brock M (ed). Intracranial pressure II. Springer, Berlin Heidelberg New York, 79-81.

Lopez-Ibor B, Garcia-Sola R, Hernandez C, Moro M, Casado E (1982). ICP monitoring during the first week of life in the preterm infant. In: Monogr Paediatr, Karger, Basel, 15: 134-138.

Lorber J, Priestly BL (1981). Children with large heads: a practical approach to diagnosis in 557 children, with special reference to 109 children with megalencephaly. Develop Med Child Neurol 23: 494-504.

Lorenzo AV, Page LK, Watters GV (1970). Relationship between cerebrospinal fluid formation, absorption and pressure in human hydrocephalus. Brain 93: 679-692.

Lou HC, Skov H, Pederson H (1979). Low cerebral blood flow: a risk factor in the neonate. J Pediatr 95: 606-??

Lundberg N (1960). Continuous recording and control of ventricular fluid pressure in neurosurgical practice. Acta Psych Neurol Scand 36, suppl 149: 1-193.

Lundberg $N$ (1972). Clinical indications for measurement of ICP. In: Brock M, Dietz H (ed). Intracranial pressure. Springer, Berlin Heidelberg New York, 369-371.

Lundberg N, Kjällquist A, Kullberg G, Ponten U, Sundbärg G (1974). Non operative management of intracranial hypertension. In: Kraijenbuhl $\mathrm{H}$ (ed). Advances and Technical standards in Neurosurgery I. Springer, Wien, 3-59.

Lups S, Haan AMFH (1954). The cerebrospinal fluid. Elsevier Publishing Comp, Amsterdam, 30-47.

Maas AIR, Jong DA de (1986a). The Rotterdam Teletransducer: State

of the device. Acta Neurochirurgica 79: 5-12.
Maas AIR, Jong DA de (1986b). The Rotterdam teletransducer: Telemetric epidural pressure monitoring - Results of comparitive EDP-VFP measurements. In: Miller JD, Teasdale GM, Rowan JO, Galbraith SL, Mendelow AD (ed). Intracranial pressure VI. Springer, Berlin Heidelberg New York Tokyo, 207-212.

Mackay RS, Marg E, Oechsli R (1960). Automatic tonometer with exact theory: various biological applications. Science 131: 1668-1669.

Mackay RS (1964). The application of physical transducers to intracavity pressure measurement with special reference to tonometry. Med Electron Biol Engng 2: 3-19.

Magnaes B (1976a). Body position and cerebrospinal fluid pressure. Part 1: clinical studies on the effect of rapid postural changes. J Neurosurg 44: 687-697.

Magnaes B (1976b). Body position and cerebrospinal fluid pressure. Part 2: Clinical studies on orthostatic pressure and the hydrostatic indifferent point. J Neurosurg 44: 698-705.

Majors R, Schettini A, Mahig J, Nevis AH (1972). Intracranial pressures measured with the co-planar pressure transducer. Med Biol Eng 10: $724-733$.

Marmarou A, Shulman K, LaMorgese J (1975). Compartmental analysis of compliance and outflow resistance of the cerebrospinal fluid system. J Neurosurg 43: 523-534.

Marmarou A, Shulman K, Rosende RM (1978). A non-linear analysis of the cerebrospinal fluid system and intracranial pressure dynamics. J Neurosurg 48: 332-344.

Maytal J, Alvarez LA, Elkin CM, Shinnar S (1987). External hydrocephalus: Radiologic spectrum and differentiation from cerebral atrophy. AJNR 8: 271-278.

McCullough DC (1980). A critical evaluation of continuous intracranial pressure monitoring in pediatric hydrocephalus. Child's Brain 6: 225241.

McIntosh N (1983). The monitoring of critically ill neonates. J Med Eng Technol 7: 121129. 
Mehta A, Wright BM, Shore C (1988). Clinical fontanometry in the newborn. Lancet I: 754756.

Mendelow AD, Rowan JO, Murray L, Kerr AE (1983). A clinical comparison of subdural screw pressure measurements with ventricular pressure. J Neurosurg 58: 45-50.

Menke JA, Miles R, McIlhany M, Bashiru M, Chua C, Schwied E, Menten TG, Khanna NN (1982). The fontanelle tonometer: a noninvasive method for measurement of intracranial pressure. J Pediatr 100: 960-963.

Ment LR, Duncan CC, Geehr R (1981). Benign enlargement of the subarachnoid spaces in the infant. J Neurosurg 54: 504-508.

Merritt HH, Fremont-Smith F (1937). The cerebrospinal fluid. Saunders Company, Philadelphia London, 45-50, 73-74.

Mickell JJ, Ward JD (1984). Evaluation and treatment of increased intracranial pressure. In: Kelly VC (ed). Practice of pediatrics. Harper and Row Publish, Philidelphia, 1-37.

Mikati MA, Dudin GE, Der Kaloustian VM, Benson PF, Fenson AH (1982). Maple syrup urine disease with increased intracranial pressure. Am J Dis Child 136: 642-643.

Miller JD, Garibi J, Pickard JD (1973). Induced changes of cerebrospinal fluid volume: effects during continuous monitoring of ventricular fluid pressure. Arch Neurol 28: 265269.

Miller JD, Becker DP, Ward JD (1977). Significance of intracranial hypertension in severe head injury. J Neurosurg 47: 503-516.

Miller JD (1978). Intracranial pressure monitoring. Br J Hosp Med 19: 497-503.

Miller JD, Butterworth JF, Gudeman SK, Faulkner JE, Choi SC, Selhorst JB, Harbison JW, Lutz HA, Young HF, Becker DP (1981). Further experience in the management of severe head injury. J Neurosurg 54: 289-299.

Miller JD (1983). Significance and management of intracranial hypertension in head injury. In: Ishii S, Nagai H, Brock M (ed). Intracranial pressure V. Springer, Berlin Heidelberg, 44-53.
Miller JD (1985). Intracranial pressure monitoring. Arch Neurol 42: 1191-1193.

Minns RA (1976). Ventricular pressure monitoring of spina bifida children. Dev Med Child Neurol 18 suppl.37: 166-167.

Minns RA (1977). Clinical application of ventricular pressure monitoring in children. $\mathrm{Z}$ Kinderchir 22: 430-443.

Minns RA (1984). Intracranial pressure monitoring. Arch Dis Child 59: 486-488.

Minns RA, Engleman HM (1988). The use of CSF pressure recordings in acute purulent meningitis. Z Kinderchir 43 suppl.II: 28-29.

Modic MT, Kaufman B, Bonstelle CT, Tomsick TA, Weinstein MA (1981). Megalocephaly and hypodense extracerebral fluid collections. Neuroradiology 141: 93-100.

Moses RA, Marg E, Oechsli R (1962). Evaluation of the basic validity and clinical usefulness of the Mackay-Marg tonometer. Invest Opthal 1: 78-85.

Moss E, McDowall DG (1979): Monitoring of intracranial pressure. In: Trubuhovich RV (ed). Management of Intracranial disasters. Little, Brown and Co., Boston, Int Anaesth Clin 17: 375-390.

Munro D (1928). Cerebrospinal fluid pressure in the newborn. JAMA 90: 1688-1689.

Myerberg DZ, York C, Chaplin ER, Gregory GA (1980). Comparison of non-invasive and direct measurements of intracranial pressure. Pediatrics 65: 473-476.

Myerberg DZ (1981). Measurement of intracranial pressure. Pediatrics 67: 747-748.

Naidich ThP, Epstein F, Lin JP, Kricheff II, Hochwald GM (1976). Evolution of pediatric hydrocephalus by computed tomography. Radiology 119: 337-345. 
Narayan RK, Kishore PRS, Becker DP, Ward JD, Enas GG, Greenberg RP, Domingues da Silva A, Lipper MH, Choi SC, Mayhall CG, Lutz III HA, Young HF (1982). Intracranial pressure: to monitor or not to monitor ? A review of our experience with severe head injury. J Neurosurg 56: 650-659.

Nellhaus G (1968). Head circumference from birth to eighteen years. Pediatrics 41: 106-114.

Newton RW (1987). Intracranial pressure and its monitoring in childhood: a review. J Royal Soc Med 80: 566-570.

Nickel RE, Gallenstein JS (1987). Developmental prognosis for infants with benign enlargement of the subarachnoidal spaces. Dev Med Child Neurol 29: 181-186.

Nornes H, Serck-Hansen F (1970). Miniature transducer for intracranial pressure monitoring in man. Acta Neurol Scand 46: 203-214.

Nornes H, Magnaes B (1971). Supratentorial epidural pressure recorded during posterior fossa surgery. J Neurosurg 35: 541-549.

Norrell $\mathrm{H}$, Wilson C, Howieson J, Megison L, Bertan V (1969). Venous factors in infantile hydrocephalus. J Neurosurg 31: 561-569.

Numoto M, Slater JP, Donaghy RMP (1966). An implantable switch for monitoring intracranial pressure. Lancet I: 528 .

Numoto M, Wallman JK, Donaghy RMP (1973). Pressure indicating bag for monitoring intracranial pressure. Technical note. J Neurosurg 39: 784-787.

Nussbaum E, Galant SP (1983). Intracranial pressure monitoring as a guide to prognosis in the nearly drowned, severaly comatose child. J Pediatr 102: 215-218.

Ostrup RC, Luerssen ThG, Marshall LF, Zornow MH (1987). Continuous monitoring of intracranial pressure with a miniaturized fiber-optic device. J Neurosurg 67: 206-209.

Papile LA, Burstein J, Burstein R, Koffer H (1978). Incidence and evolution of subependymal and intraventricular hemorrhage: a study of infants with birth weights less than 1500 gr. J Pediatr 92: 529-534.
Paraicz E, Vajda J (1975a). Fortlaufende Registrierung der intraventrikulären Druckwellen bei Hydrocephalus im Säuglingsalter. Neurochirurgia 18: 43-51.

Paraicz E, Vajda J (1975b). Arterial and respiratory influences on intracranial pressure in infancy. Surg Neurol 4: 361-366.

Paraicz E (1978). A-waves in infantile and children's hydrocephalus. J Neurosurg Sci 22: 169-171.

Paraicz E (1982). Introduction. In: Monogr Paediatr, Karger, Basel, 15: 1-7.

Peabody JL, Schneider H, Huch R, Huch A (1981). A new device for non-invasive measurements of intracranial pressure (ICP) in newborns. J Perinat Med 9, suppl 1: 125-128.

Perlman JM, Volpe JJ (1983). Suctioning in the preterm infant: effects on cerebral bloodflow velocity, intracranial pressure, and arterial blood pressure. Pediatrics 72: 329-334.

Philip AGS (1979). Non-invasive monitoring of intracranial pressure. A new approach for neonatal clinical pharmacology. Clin Perinat 6: 123-137.

Philip AGS, Long JG, Donn SM (1981). Intracranial pressure. Sequential measurements in full-term and preterm infants. Am J Dis Child 135: 521-524.

Picton-Warlow CG, Robinson RJ (1970). Evaluation of a method of indirect measurement of intracranial pressure in infants. Dev Med Child Neurol 12: 507-511.

Plandsoen WCG, Jong DA de, Eijndhoven JHM van, Stroink H (1986a). Non-invasive ICP monitoring in the anterior fontanelle in newborn children, using the Rotterdam Teletransducer. Clin Neurol Neurosurg 88: 321-322.

Plandsoen WCG, Jong DA de, Maas AIR, Stroink H, Eijndhoven JHM van (1986b). The pressure depth curve in anterior fontanelle pressure monitoring. In: Miller JD, Teasdale GM, Rowan JO, Galbraith SL, Mendelow AD (ed). Intracranial Pressure VI. Springer, Berlin Heidelberg New York Tokyo, 193-198. 
Plandsoen WCG, Jong DA de, Maas AIR, Stroink H, Avezaat CJJ (1987). Fontanelle pressure monitoring with the Rotterdam Teletransducer; a reliable technique. Med Progr Technol 13: 21-27.

Plandsoen WCG, Jong DA de, Maas AIR, Delwel EJ, Avezaat CJJ, Loonen MCB (1988). The Rotterdam Teletransducer (RTT). A noninvasive method for the measurement of intracranial pressure in adults and newborns. Clin Neurol Neurosurg 90: 185.

Plandsoen WCG, Avezaat CJJ, Jong DA de (1989a). Anterior Fontanelle Pressure (AFP) Monitoring: technique and clinical results. Clin Neurol Neurosurg 91: 98.

Plandsoen WCG, Jonge DA de, Avezaat CJJ, Eijndhoven JHM van, Loonen MCB, Mulder PGH (1989b). Non-invasive monitoring of ICP in infants. In: Hoff JT and Betz AL (ed). Intracranial pressure VII. Springer, Berlin Heidelberg New York London Paris Tokyo Hong Kong, 91-94.

Popich GA, Smith DW (1972). Fontanels: range of normal size. J Pediatr 80: 749-752.

Portnoy HD, Croissant PD (1978). Megalencephaly in infants and children. Arch Neurol 35: 306-316.

Powell MP, Crockard HA (1985). Behavior of an extradural pressure monitor in clinical use. J Neurosurg 63: 745-749.

Pozo JL, Crockard HA, Ransford AO (1984). Basilar impression in osteogenesis imperfecta. J Bone Joint Surg Br 66: 233-238.

Purin VR (1964). 'Measurement of the cerebrospinal fluid pressure in the infant without puncture. A new method.' Pediatrya 43: 82-84.

Quigley HA, Langham ME (1975). Comparative intra-ocular pressure measurements with the pneumotonograph and Goldman tonometer. Am J Ophthalmol 80: 266-273.

Quincke H (1878). Ueber den Druck in Transsudaten. Deutches Archiv für Klin Med, Band XXI, 464-468.
Quincke H (1891a). Die Lumbalpunktion des Hydrocephalus. Berl Klin Wochenschr 28:929933, 965-968.

Quincke H (1891b). Ueber Hydrocephalus. Verhandl X. Congresses Inn Med 40: 321-340.

Raju TNK, Vidyasagar D (1980). Intracranial pressure studies in acutely ill neonates using a non-invasive technique: a three year experience. In: Shulman K, Marmarou A, Miller JD, Becker DP, Hochwald GM, Brock M (ed). ICP IV. Springer, Berlin Heidelberg New York, 392394.

Raju TNK, Vidyasagar D, Papazafiratou C, (1980). Intracranial pressure monitoring in the neonatal ICU. Critical Care Med 8: 575-581.

Raju TNK, Vidyasagar D, Papazafiratou C (1981). Cerebral perfusion pressure and abnormal intracranial pressure waveforms: their relation to outcome in birth asfyxia. Crit Care Med 6: 449-453.

Raju TNK, Doshi UV, Vidyasagar D (1982a). Cerebral perfusion pressure studies in healthy preterm and term newborn infants. J Pediatr 100: 139-142.

Raju TNK, Bhat R, Vidyasagar D (1982b). Age-related difference in cerebral perfusion pressure response to acute hypoxia in neonatal puppies. Biol Neonate 41: 258-264.

Rand BO, Ward AA, White LE (1966). The use of the twist drill to evaluate head trauma. J Neurosurg 25: 410-415.

Renier D, Sainte-Rose C, Marchac D, Hirsch JF (1982). Intracranial pressure in craniostenosis. J Neurosurg 57: 370-377.

Riechert T, Heines KD (1950). Ueber zwei Untersuchungsmethoden zur Beurteilung der Hirn durchblutung. Nervenarzt 21: 9-16.

Risberg J, Lundberg N, Ingvar DH (1969). Regional cerebral blood volume during acute transient rises of the intracranial pressure (plateau waves). I Neurosurg 31: 303-310. 
Roberts PA, Fullenwider C, Stevens FA, Pollay $\mathbf{M}$ (1983). Experimental and clinical experience with a new solid state intracranial pressure monitor with in vivo zero stability. In: Ishii S, Nagai H, Brock M (ed) ICP V. Springer, Berlin Heidelberg New York Tokyo, 104-105.

Robertson WC, Gomez MR (1978). External hydrocephalus. Arch Neurol 35: 541-544.

Robertson WC, Chun RWM, Orrison WW, Sackett JF (1979). Benign subdural collections of infancy. J Pediatr 94: 382-385.

Robinson RO, Rolfe P, Sutton P (1977). Noninvasive method for measuring intracranial pressure in normal newborn infants. Dev Med Child Neurol 19: 305-308.

Rochefort MJ, Rolfe P, Wilkinson AR (1987). New fontanometer for continuous estimation of intracranial pressure in the newborn. Arch Dis Child 62: 152-155.

Ropper AH (1985). In favor of intracranial pressure monitoring and aggressive therapy in neurologic practice. Arch Neurol 42: 1194-1195.

Rosman NP, Shands KN (1978). Hydrocephalus caused by increased intracranial venous pressure: A clinicopathological study. Ann Neurol 3: 445-450.

Rosner MJ, Becker DP (1976). ICP Monitoring: complications and associated factors. Clin Neurosurg 23: 494-519.

Rosner MJ, Becker DP (1983). The etiology of plateau waves: a theoretical model and experimental observations. In: Ishii S, Nagai H, Brock M (ed). Intracranial pressure V. Springer, Berlin Heidelberg, 301-305.

Rothballer AB (1963). Continuous recording of intracranial pressure in man and animals. Presented at meeting of Harvey Cushing Society Philadelphia, Penn, april.

Rothenberger A, Brandl H (1980a). Diagnostik des Subduralergusses im Kindesalter. Kinderarzt 11: 1073-1075.
Rothenberger A, Brandl H (1980b). Subdural effusions in children under two years - clinical and computer-tomographical data. Neuropaediatrie 11: 139-150.

Rowan JO, Mendelow AD, Murray L, Kerr A (1983). Clinical comparison of intracranial pressure measurements provided by subdural screws and ventricular catheter. In: Ishii $S$, Nagai H, Brock M (ed). Intracranial pressure V. Springer, Berlin Heidelberg New York Tokyo, 110-115.

Rubin RC, Henderson ES, Ommaya AK, Walker MD, Rall DP (1966). The production of cerebrospinal fluid in man and its modification by acetazolamide. J Neurosurg 25: 430436.

Rylander HG, Taylor HL, Wissinger JP, Story JL (1976). Chronic measurement of epidural pressure with an induction-powered oscillator transducer. J Neurosurg 44: 465-478.

Sahar A (1978). Pseudohydrocephalus-megalocephaly, increased intracranial pressure and widened subarachnoid space. Neuropädiatrie 9: 131-139.

Sainte-Rose C, Renier D, Pierre-Kahn A, Hirsch JF (1980). Apport de l'engistrement continue de la pression intra-cranienne en neurochirurgie pédiatrique. Rev Pédiatrie 9: 539552.

Sainte-Rose C, LaCombe J, Pierre-Kahn A, Renier D, Hirsch JF (1984). Intracranial venous sinus hypertension: cause or consequence of hydrocephalus in infants? J Neurosurg 60: 727-736.

Salmon JH, Hajjar W, Bada HS (1977). The fontogram: A non-invasive Intracranial Pressure Monitor. Pediatrics 60: 721-725.

Sarnaik AP, Preston G, Lieh-lai M, Eisenbrey AB (1985). Intracranial pressure and cerebral perfusion pressure in near-drowning. Crit Care Med 13: 224-227.

Scanarini M, Ori C, Pittoni G, Longatti PL, Gerosa M, Carteri A (1982). Chloral Hydrate and intracranial pressure at pediatric age. In: Monogr Paediatr 15: 67-69. 
Schettini A, McKay L, Majors R, Mahig J, Nevis AH (1971). Experimental approach for monitoring surface brain pressure. J Neurosurg 34: 38-47.

Schettini A, Walsh EK (1974). Experimental identification of the subarachnoid and subpial compartments by intracranial pressure measurements. J Neurosurg 40: 609-616.

Schettini A, Walsh EK (1975). Simultaneous pressure depth measurements of the intracranial system made epidurally. In: Lundberg N, Ponten U, Brock M (ed). Intracranial Pressure II. Springer, Berlin, 409-413.

Schulz HP, Hilton GF, McEwen WK (1963). Fast-acting applanation tonometers. Arch Ophthalm 69: 77-81.

Shapiro K, Marmarou A, Shulman K (1980). A method for predicting PVI in normal patients. In: Shulman K, Marmarou A, Miller JD, Becker DP, Hochwald GM, Brock M (ed). Intracranial pressure IV, Springer, Berlin Heidelberg New York, 85-87.

Shapiro K, Marmarou A, Portnoy H (1984). Hydrocephalus. Raven press, New York, pp 401.

Shulman K, Ransohoff J (1965). Sagittal sinus venous pressure in hydrocephalus. J Neurosurg 23: $169-173$.

Shulman K, Marmarou A (1971). Pressurevolume considerations in infantile hydrocephalus. Dev Med Child Neurol 13,suppl 25: 9095.

Sidbury JB (1920). The importance of lumbar puncture in intracranial hemorrhage of the newborn. Report of a case with recovery. Arch Pediatr 37: 545-553.

Silver W, Kuskin L, Goldenberg L (1970). Bulging anterior fontanelle. Sign of congestive heartfailure in infants. Clin Pediatr 9: 42-43.

Skjodt Th, Teisen HG, Torfing KF, Blicher $\mathrm{J}$ (1988). Intra- and interobserver variation in CT-evaluated cerebral atrophy. Fortschr Rontgenstr 149, 6: 594-595.
Sklar FH, Beyer CW, Ramanathan M, Kemp Clark W (1980). Servo controlled lumbar infusions in childhood. J Neurosurg 52: 87-98.

Smith RW, Alksne JF (1976). Infections complicating the use of external ventriculostomy. J Neurosurg 44: 567-570.

Smyth CN (1957). The guard-ring tocodynamometer. J Obstet Gynaec Brit Emp 64: 5966.

Souza SW de, Ross J, Milner RDG (1976). Alterations in head shape of newborn infants after caesarean section or vaginal delivery. Arch Dis Child 51: 624-627.

Steinbok P, Hall JK, Flodmark O (1989). Hydrocephalus in achondroplasia: the possible role of intracranial venous hypertension. J Neurosurg 71: 42-48.

Stuart G, Merry G, Smith J, Yelland JDN (1983). Severe head injury managed without intracranial pressure monitoring. J Neurosurg 59: 601-605.

Sundbärg G, Kjällquist A, Lundberg N, Ponten U (1972). Complications due to prolonged ventricular fluid pressure recording in clinical practice. In: Brock M, Dietz H (ed). Intracranial Pressure. Springer, Berlin Heidelberg New York, 348-352.

Sundbärg G, Nornes H (1972). Simultaneous recording of the epidural and ventricular fluid pressure. In: Brock M, Dietz H (ed). Intracranial pressure. Springer, Berlin Heidelberg New York, 46-50.

Symon L, Dorsch NWC (1975). Use of longterm intracranial pressure measurement to assess hydrocephalic patients prior to shunt surgery. J Neurosurg 42: 258-273.

Takagi H, Miyasaka Y, Morii S, Ohwada T, Yada K (1986). Clinical experience of 780 cases of postoperative ICP monitoring. In: Miller JD, Teasdale GM, Rowan JO, Galbraith SL, Mendelow AD (ed). Intracranial pressure VI. Springer, Berlin Heidelberg New York Tokyo, 695700 . 
Tindall GT, McGraw CP, Wendenburg HO, Peel HH (1972). Evaluation of a subdural pressure transducer. Technical note. J Neurosurg 37: 117-121.

Toonder M (1983). Als je begrijpt wat ik bedoel. Het kukel. Herdruk. De Bezige Bij, Amsterdam, pp 159.

Tsipouras P, Barabas G, Matthews WS (1986). Neurologic correlates of osteogenesis imperfecta. Arch Neurol 43: 150-152.

Turner JM, Gibson RM, McDowall DG, Nahhas F (1975). Further experiences with extradural pressure monitoring. In: Lundberg $\mathrm{N}$, Ponten U, Brock M (ed). Intracranial Pressure II. Springer, Berlin, 397-402.

Turner JM, McDowall DG (1976). The measurement of intracranial pressure. $\mathrm{Br} \mathrm{J}$ Anaesth 48: 735-740.

Venes J (1979). B-waves - A reflection of cardiorespiratory or cerebral nervous systems rhythm? Child's Brain 5: 352-360.

Vidyasagar D, Raju TNK (1977). A simple non-invasive technique of measuring intracranial pressure in the newborn. Pediatrics 59: 957961.

Vidyasagar D (1978). Intracranial pressure monitoring as an adjunct to the management of sick neonates. In: Kumar S, Rathi M (ed). Perinatal Medicine. Pergamon Press, Oxford, 151-163.

Vidyasagar D, Raju TNK, Chiang J (1978). Clinical significance of monitoring anterior fontanel pressure in sick neonates and infants. Pediatrics 62: 996-999.

Volpe JJ (1987). Intracranial hemorrhage: periventricular-intraventricular hemorrhage of the premature infant. In: Neurology of the Newborn, 2nd edition. Major problems in clinical pediatrics, Vol 22. W.B. Saunders Company, Philadelphia Londen, 311-361.

Volpe JJ (1989a). Intraventricular hemorrhage in the premature infant - Current concepts. Part I. Ann Neurol 25: 3-11.
Volpe JJ (1989b). Intraventricular hemorrhage in the premature infant - Current concepts. Part II. Ann Neurol 25: 109-116.

Vries JK, Becker DP, Young HF (1973). A subarachnoid screw for monitoring intracranial pressure. Technical note. J Neurosurg 39: 416419.

Walsch P, Logan WJ (1983). Continuous and intermittent measurement of intracranial pressure by the Ladd monitor. J Pediatr 102: 439442.

Wealthall SR, Smallwood R (1974). Methods of measuring intracranial pressure via the fontanelle without puncture. J Neurol Neurosurg Psychiatry 37: 88-96.

Weaver DD, Christian JC (1980). Familial variation of head size and adjustment for parental head circumference. J Pediatr 96: 990994.

Weinstein RA (1976). The design of pressure monitoring devices: Infection control considerations. Med Instrum 10: 287-290.

Welch K (1978). Normal pressure hydrocephalus in infants and children: a reappraisal. $Z$ Kinderchir 25: 319-324.

Welch $\mathrm{K}$ (1980). The intracranial pressure in infants. J Neurosurg 52: 693-699.

Wentzler E (1922a). Ein apparat zur Messung des Schädelinnendruckes an der Fontanelle des Säuglings. Arch Kinderheilk 70: 241-245.

Wentzler E (1922b). Tonometer für Messung des Schädelinnendruckes beim Säugling. Reichspatentamt Patentschrift 363101, Klasse 30 , gruppe 4.

West CE, Capella JA, Kaufman HE (1972). Measurement of intraocular pressure with a pneumatic applanation tonometer. Am J Ophthal 74: 505-509.

Whitelaw AGL, Wright BM (1982). A pneumatic applanimeter for intracranial pressure measurements. J Physiol 336: 3p-4p. 
WHO: Recommended definitions, terminology and format for statistical tables related to the perinatal period and use of a new certificate for cause of perinatal deaths (1977). Acta Obstet Gynaecol Scand 56: 247-253.

Wild K von, Pörksen C (1980). Non-invasive technique for monitoring intracranial pressure via the fontanelle in premature infants and newborns with hydrocephalus. Z Kinderchir 31: 348-353.

Wild $\mathrm{K}$ von (1981). The evaluation of noninvasive fibre-optical monitoring of intracranial pressure (ICP) over the fontanelle. Z Kinderchir 34: 147-149.

Wild K von, Pörksen C, Anupöld V (1982). Nicht-invasive kontinuierliche Druckmessung über die offenen Fontanelle mit einem fiberoptischen Sensor. In: Loewenich von (ed). Paediatrische Intensiv Medizin III, Intensiv $\mathrm{Me}-$ dizin. Notfall Medizin. Anasthesiologie, band 36. Thieme Verlag, Stuttgart New York, 7787.

Williams J, Hirsch NJ, Corbet AJS, Rudolph AJ (1977). Postnatal head shrinkage in small infants. Pediatrics 59: 619-622.

Winn HR, Dacey RG, Jane JA (1977). Intracranial subarachnoid pressure recording: experience with 650 patients. Surg Neurol 8: 4147.
Wulff HR (1981). Rational diagnosis and treatment, 2nd edition. Blackwell scientific publications, Oxford London Edinburgh Boston Melbourne, 43-45.

Wyler AR, Kelly W (1972). Use of antibiotics with external ventriculostomies. J Neurosurg 37: 185-187.

Yamada H (1983). Pediatric Cranial Computed Tomography. Igaku Shoin, Tokyo New York, 116.

Young B (1979). Hydrocephalus and elevated intracranial venous pressure. Child's Brain 5: 73-80.

Zervas NT, Cosman ER, Cosman BJ (1977). A pressure-balanced radio-telemetry system for the measurement of intracranial pressure. A preliminary design report. J Neurosurg 47: 899911.

Zierski J (1980). Extradural, ventricular and subdural pressure recording comparative clinical study. In: Shulman K, Marmarou A, Miller JD, Becker DP, Hochwald GM, Brock M (ed). Intracranial pressure IV. Springer, Berlin Heidelberg New York, 371-376. 


\section{ABBREVIATIONS}

\begin{tabular}{|c|c|c|c|}
\hline $\mathrm{AC}$ & alternating current & LP & lumbar pressure \\
\hline AFP & anterior fontanelle pressure & $\mathrm{MHz}$ & megahertz \\
\hline $\mathrm{AFP}_{\mathrm{c}}$ & anterior fontanelle pressure & $\mathrm{ml}$ & milliliter \\
\hline & during continuous monitoring & $\mu \mathrm{m}$ & micrometer \\
\hline AFP score & average of three PDC's & $\mathrm{mm}$ & millimeter \\
\hline $\mathrm{C}$ & compliance $=\mathrm{dV} / \mathrm{dP}$ & $1 \mathrm{mmHg}$ & $=1,36 \mathrm{cmH}_{2} \mathrm{O}$ \\
\hline $\mathrm{CBF}$ & cerebral blood flow & $\min$ & minute \\
\hline $\mathrm{CBV}$ & cerebral blood volume & NS & not significant \\
\hline $\mathrm{cm}$ & centimeter & $\mathrm{P}$ & pressure \\
\hline CSF & cerebrospinal fluid & $\mathrm{Pa}$ & plateau on ascending limb of \\
\hline CSFP & cerebrospinal fluid pressure & & pressure depth curve \\
\hline d & 'change in' & $\mathrm{Pb}$ & plateau on descending limb of \\
\hline $\mathrm{E}$ & elastance $=\mathrm{dP} / \mathrm{dV}$ & & pressure depth curve \\
\hline EDP & epidural pressure & PDC & pressure depth curve \\
\hline $\mathrm{Fi}_{i}$ & CSF formation rate $(\mathrm{ml} / \mathrm{min})$ & PVI & pressure volume index $(\mathrm{ml})$ \\
\hline Fr & resonance frequency & Ro & CSF outflow resistance \\
\hline $\mathrm{HF}$ & high frequency & & $(\mathrm{mmHg} / \mathrm{ml} / \mathrm{min})$ \\
\hline $\mathrm{Hg}$ & mercury & RT'T & Rotterdam Teletransducer \\
\hline $\mathrm{H}_{2} \mathrm{O}$ & water & $\mathrm{SD}$ & standard deviation \\
\hline $\mathrm{Hz}$ & hertz $(1 \mathrm{~Hz}=1 \mathrm{cycle} / \mathrm{second})$ & SS & sagittal sinus \\
\hline ICP & intracranial pressure & $\mathrm{V}$ & volume \\
\hline IVH & intraventricular haemorrhage & VFP & ventricular fluid pressure \\
\hline
\end{tabular}




\section{NAWOORD}

Hoewel het nawoord niet het belangrijkste deel van een proefschrift is, behoort het met de stellingen tot de meest gelezen fragmenten ervan. Met opzet is het woord 'helaas' weggelaten omdat ik graag naambekendheid wil geven aan degenen die in deze fase van mijn leven en werken van betekenis zijn geweest.

Zeergeleerde Van Eijndhoven, beste John, jij hebt indertijd mijn wetenschappelijke belangstelling gewekt voor de niet-invasieve intracraniële drukmeting. Daarvoor en voor de begeleiding door de jaren heen dank ik je zeer. Jouw adviezen en de warme steun van je vrouw Truus hebben een wezenlijke rol gespeeld bij de totstandkoming van dit proefschrift. Aan de vele bezoeken bij jullie thuis bewaar ik een warme herinnering.

'Inventor', D.A. de Jong, waarde Dirk, jouw vinding vormt de basis van dit werk en je leerde mij hoe het apparaat te hanteren. Onze samenwerking heeft de fontaneltoepassing van de Rotterdam Teletransducer ruime bekendheid gegeven. Voor dit alles ben ik je zeer dankbaar.

Bijzondere dank ben ik verschuldigd aan mevrouw dr. M.C.B. Loonen. Lieve Christa, jouw hartelijke steun en stimulerende begeleiding gaven mij tijdens de moeilijke momenten de energie om door te gaan. Zonder onze vruchtbare discussies zou dit werk nooit zijn uitgegroeid tot een dissertatie. Dat je bovendien mijn co-promotor bent, beschouw ik als een grote eer.

Hooggeleerde Avezaat, beste Cees, ik weet niet of je altijd even blij bent geweest met mijn constante aandrang om de hoofdstukken te beschouwen. Je kritische benadering is het werk ten goede gekomen. Door jou heb ik een beter inzicht gekregen in 'ICP and craniospinal dynamics'. Voor dit alles ben ik je zeer erkentelijk.

Hooggeleerde Van Crevel, beste Hans, voor de vanzelfsprekendheid waarmee je mij de ruimte gaf om het Rotterdamse werk te voltooien, ben ik je veel dank verschuldigd. Hooggeleerde Barth, beste Peter, en geleerde Geesink, beste Huibert, als direkte collega's op de kinderneurologie hebben jullie, door zo nu en dan mijn werk over te nemen, een belangrijke bijdrage geleverd aan de afronding van mijn proefschrift. Mijn dank is groot.

Heel veel dank ben ik verschuldigd aan Jacquelien van der Vlies, die immer bereid was de laatste correcties 'voor morgen' aan te brengen. Lieve Jacquelien, jouw vriendschap heeft me meerdere malen over een zekere drempel geholpen.

Geleerde Mulder, beste Paul, jij voerde met veel geduld de statistische bewerkingen uit. Ik dank je voor de bereidheid waarmee je dit hebt gedaan. Voor de vanzelfsprekendheid waarmee de heer C.J. Jongkind en ir. A. Boogaard het computerprogramma hebben geschreven, ben ik jullie, Cees en Arie dankbaar.

De normaalwaarden zijn verkregen dankzij de welwillende medewerking van prof.dr. H.C.S. Wallenburg, verbonden aan de afdeling gynaecologie/verloskunde van het Academisch Ziekenhuis Dijkzigt, de jeugdartsen mevr. drs. R.S. Boeken KrugerMangunkusumo (thans verbonden aan de provinciale Kruisvereniging Zuid-Holland) en drs. R.E. Juttmann van de afdeling jeugdgezondheidszorg Rotterdam, de mede- 
werkers van het consultatiebureau Zevenkamp, de kinderen die voor dit doel werden gemeten en hun ouders die daarvoor toestemming gaven.

De tekeningen zijn vervaardigd door mevr. G.J.H. Wentink. Beste Gerdie, daarvoor mijn hartelijke dank.

De medewerkers van de afdeling medische fotografie en illustratie van het AMC verzorgden de afdrukken van de figuren en de aanpassingen van enkele foto's, reeds eerder vervaardigd door het AVC, Rotterdam.

De medewerkers van de afdelingen 6 Zuid en II-3e van respectievelijk het Academisch Ziekenhuis Dijkzigt en het Sophia Kinderziekenhuis dank ik voor hun constructieve bijdragen.

De Engelse tekst is gecorrigeerd door mevr. E.C. Dorling.

De voltooiing van dit proefschrift biedt mij tevens de gelegenheid een woord van dank te richten aan de hoogleraren van de Erasmus Universiteit Rotterdam, die een belangrijke bijdrage hebben geleverd an mijn medische scholing, met name dank ik mijn opleider in de neurologie prof.dr. A. Staal.

Aan alle betrokkenen, met name genoemd of onvermeld, mijn hartelijke dank.

Jan, door jouw liefdevolle aandacht was de last de laatste maanden bepaald niet zwaar. 


\section{Curriculum Vitae}

De schrijfster van dit proefschrift werd 17 januari 1953 geboren te Rotterdam. In 1972 deed zij eindexamen gymnasium-beta en ving in ditzelfde jaar de studie geneeskunde aan aan de Medische Faculteit van de

Erasmus Universiteit Rotterdam. December 1979 voltooide zij de opleiding tot arts. In 1980 was zij algemeen assistente interne geneeskunde in het Havenziekenhuis te Rotterdam. Van 1981 tot 1986 specialiseerde zij zich tot neuroloog met aantekening klinische neurofysiologie. Opleiders waren respectievelijk prof.dr. A. Staal (neurologie, Academisch Ziekenhuis Dijkzigt, Rotterdam), prof.dr. M. de Vlieger en dr. K. Mechelse (klinische neurofysiologie, Academisch Ziekenhuis Dijkzigt, Rotterdam) en dr. W.A.

Nolen (psychiatrie, Psychiatrisch Centrum Bloemendaal te Den Haag).

Van januari $1986 \mathrm{t} / \mathrm{m}$ februari 1988 werkte zij als junior-specialist op de afdeling neurochirurgie van het Academisch Ziekenhuis Dijkzigt te Rotterdam (prof.dr. C.J.J. Avezaat en prof.dr. R. Braakman) met als voornaamste taak de zorg voor de afdeling kinderneurochirurgie in het

Sophia Kinderziekenhuis te Rotterdam. Hier verrichtte zij het voornaamste deel van het onderzoek.

Vanaf 1 maart 1988 is zij werkzaam als kinderneuroloog in het Academisch Ziekenhuis bij de Universiteit van Amsterdam (afdelingshoofd prof.dr. P.G. Barth). $\mathrm{Zij}$ is als stafmedewerkster verbonden aan de afdelingen neurologie (prof.dr. H. van Crevel) en kindergeneeskunde (prof.dr. C.J. de Groot). 
